\title{
29th Meeting of the Canadian Congress of Neurological Sciences
}

\section{PLATFORM SESSIONS}

\section{Tuesday, June 28}

\section{Afternoon}

A. Surgical Oncology

B. Cerebrovascular Diseases

C. Neuromuscular Disease

D. Pediatric Neurology

\section{Wednesday, June 29}

\section{Morning}

E Epilepsy Surgery

F Radiosurgery

G General Neurology

H Behavioral Neurology

Afternoon

I Cerebrovascular Surgery

J General Neurosurgery

K Neurology (Movement Disorders, Headache) .....

L Neurophysiology
Al-Al0

B1-B11

C1-C9

DI-D11

E1-E6
F1-F6
G1-G6
H1-H6

I1-I11
J1-J11
K1-K11
L1-L10

\section{Platform Sessions}

\section{A. SURGICAL ONCOLOGY}

A1.

Complications and Results of Craniotomy for Radiation Necrosis Following Interstitial Brachytherapy

S.R. Shepard, M.W. McDermott, P.K. Sneed, D.A. Larson, M.D. Prado and P.H. Gutin (San Francisco, California)

Brachytherapy has been demonstrated to significantly increase survival in patients with primary glioblastoma and recurrent malignant gliomas. Approximately $40 \%$ to $50 \%$ of patients undergoing interstitial brachytherapy will require a second craniotomy for radiation necrosis. While the benefits and complications of interstitial brachytherapy are well reported in the literature, little information exists concerning the risks of repeat craniotomy after brachytherapy. We reviewed all patients who underwent craniotomy after 1-125 brachytherapy for malignant brain tumors at UCSF during the period between 1979 and 1992. During this time period 234 patients underwent craniotomy after brachytherapy and 130 of these patients had their reoperations at UCSF. These 130 patients (mean age $29.21 \pm 17.2 \mathrm{yrs}$, range 4 to 70 ) underwent a total of 152 craniotomies. In this group there were 63 patients with glioblastoma, 49 with anaplastic astrocytomas, 15 with mixed astrocytoma and oligodendroglioma, 2 with gemistocytic astrocytoma, 1 malignant ependymoma and 1 radiation induced sarcoma. The mean time from implant to craniotomy was 52.9 weeks (median 38 wks,

\section{POSTER SESSIONS}

Tuesday, June 28

Session I

1. Pediatric Neurology

2. Pediatric Epilepsy.

3. General Neurology.

PI-P9

P10-P19

P20-P36

\section{Wednesday, June 29}

Session II

1. Epilepsy

P37-P45

2. Cerebrovascular ..................................................... P46-P77

3. Neuro-Oncology ………………………………... P78-P95

4. Spine ............................................................. P96-P113

5. General Neurosurgery ....................................... P114-P121

6. Neuromuscular ................................................... PI22-P139

7. General Neurology ............................................. P140-P148

range 5.5 to 415.2 ). Mean survival from the time of implant was 148.1 weeks (median 104.9 wks, range 8.1 to $677+$ ) and mean survival from resperation was 95.2 weeks (median 58 wks, range 16 to $622+$ ). Mortality rate for the 152 craniotomies was $1.3 \%$ and morbidity rate was $15.1 \%$. On discharge $24 \%$ of patients were neurologically improved and $26 \%$ patients had a new or increased neurologic deficit. There were 9 wound infections in this group $(5.8 \%)$. The complication rate of repeat craniotomy for radiation necrosis was identical to that of all patients undergoing craniotomy at UCSF, with the exception of a slight increase in the wound infection rate. From this data we conclude that reoperation after interstitial brachytherapy has an acceptable complication rate and significantly improves overall patient survival.

\section{A2.}

\section{Arachnoid Cysts and Clinical Cerebral Herniation}

R. Moumdian, M. BojanowskI and C.J. Giroux (Montreal, Quebec)

Arachnoid cysts result either from an acquired (trauma, infection, ...) or a congenital anomaly of the cerebro-spinal fluid (CSF) circulation and the arachoid trabeculations.

They are often asymptomatic but may occasionally induce epilepsy or a neurologic syndrome by mass effect such as headache, nausea, vomiting, hemiparesis and hemianopsia.

Clinical cerebral herniation is very rare without the concomitant presence of hydrocephalus or an associated sub-dural haematoma. 
We have recently treated 3 such cases presenting with clinical cerebral herniation without hydrocephalus or sub-dural haematoma.

The 3 patients were women ranging from 50 to 66 years of age with a rapid clinical deterioration.

All 3 were operated upon on an emergency basis, with total recovery and partial re-expansion of the brain in $2 / 3$; the third remained in a vegetative state.

The above cases confirm the ball-valve mechanism by which arachnoid cysts may enlarge and present in such a dramatic fashion. Asymptomatic cysts should therefore be followed up on a regular basis.

\section{A3.}

\section{Continuous Low-Dose Oral VP-16 for Patients with Recurrent Malignant Glioma}

\section{D.S. FULTON and R.C. URTASUN (Edmonton, Alberta)}

VP-16 is effective for treatment of patients with malignant glioma when given intravenously in high doses. However, because the percentage of dividing cells in malignant glioma may be small, cell cycle specific drugs, such as VP-16 may be more effective if given continuously over a prolonged period. When prolonged oral VP- 16 is given at a dose of $50 \mathrm{mg} / \mathrm{m}^{2} /$ day, temporary interruptions of therapy are required because of myelosuppresion. In this study, the dose of $50 \mathrm{mg} /$ day was chosen in the hope that interruptions of therapy would not be required. VP-16, $50 \mathrm{mg} /$ day was given orally until the neutrophil count dropped to $<1.0$ or the platelets fell to $<75,000$ and resumed when the counts rose to normal levels. Thirty-seven patients with malignant glioma (13 aa, $17 \mathrm{gbm}, 7$ anaplastic oligo) were treated at the time of tumor progression. All had KPS $\geq 70$ at study entry. All patients had prior RT, 10 with adjuvant nitrosourea. 22 had prior nitrosourea chemotherapy for tumor recurrence, 5 had no prior chemotherapy. 16 patients were treated with VP-16 at first recurrence, 21 at 2 nd of 3 rd recurrence. All patients had CT or MR scans and clinical evaluation every 8 weeks. Median TTP was 9.0 weeks for all patients, $9.1 \mathrm{wk}$ for those treated at first progression and $9.1 \mathrm{wk}$ for those treated at 2nd or 3rd progression, $10.0 \mathrm{wk}$ for aa and $6.8 \mathrm{wk}$ for $\mathrm{gbm}$. Median survival was $23.6 \mathrm{wk}$ for all patients, 18.5 for those treated at first recurrence and $26.0 \mathrm{wk}$ for those treated at $2 \mathrm{nd}$ or 3rd progression, $38.4 \mathrm{wk}$ for aa and $14.2 \mathrm{wk}$ for $\mathrm{gbm}$. There were 6 partial responses and 8 patients with stable disease for at least 8 weeks $(R+S D=41 \%)$. The study suggests that prolonged low-dose oral VP-16 is effective for patients with malignant glioma previously treated with nitrosourea and is more effective for aa than $\mathrm{gbm}$.

\section{A4.}

\section{The Use of Photodynamic Therapy in the Treatment of Recurrent Gliomas}

\section{P.J. Muller and B.C. Wilson (Toronto, Ontario)}

Fifty patients with recurrent gliomas were candidates for palliative reoperation and photodynamic therapy [PDT]. All had failed radiation. The male/female ratio was $30 / 20$; their mean age was $40 \pm 13$ and the mean Karnofsky score was $78 \pm 15$. There were 27 with gbm, 15 with malignant astrocytomas and 8 with other gliomas. Five of the gbms arose from lower grade tumours. Photofrin $2 \mathrm{mg} / \mathrm{kg}$ was administered i.v. 12-36 hours prior to tumour resection. An argon pumped dye laser tuned to $630 \mathrm{~nm}$ light was used for cavitary photoillumination. The median photic energy was $1755 \mathrm{~J}$ and the median energy density was $45 \mathrm{~J} / \mathrm{cm}^{2}$.

There were 2 post-operative deaths - one secondary to an intracerebral hemorrhage into the tumour cavity and the other from a pulmonary embolism.

The median survival for this recurrent group from PDT was 7.2 months and the 1 and 2 year survival rates were $31 \%$ and $19 \%$, respectively; and, the median survival from first operation was 27 months with 1 and 2 year survival rates of $84 \%$ and $60 \%$, respectively. The median survival for the 27 recurrent glioblastoma patients from PDT was 6.1 months with a 1 year survival rate of $16 \%$; and, the median survival from first operation of the 22 patients with an initial diagnosis of gbm was 20 months with 1 and 2 year survival rates of $84 \%$ and $60 \%$, respectively, Further trials with higher light doses are being developed.

\section{A5.}

\section{Brachytherapy for Malignant Brain Tumours: Focus on Long-Term Survivors}

\section{Bernstein, N. Laperriere, J. Glen and S. McKenzie (Toronto, Ontario)}

Between October 1986 and June 1993, 109 patients with malignant brain tumours have been treated with stereotactic high-activity iodine- 125 implants.

For 53 patients with de-novo malignant astrocytoma treated as a boost to external radiation on one arm of a randomized study (as yet not completed), the median survival is 68 weeks. Ten patients have lived at least 2 years $(18.9 \%)$ and 7 have lived at least 3 years $(13.2 \%)$ with 5 still alive 3.5 - 6 years postimplant.

For 46 patients treated for malignant astrocytoma which recurred following surgery and external radiation (and chemotherapy in over $50 \%$ of cases), the median survival is 46 weeks post-implant and 5 patients have survived at least 2 years (10.9\%) with 2 still alive 2 and 4 years post-implant.

For 10 patients treated for single metastasis which recurred following surgery and external radiation, the median survival is 46 weeks post-implant and 4 patients have lived over 2 years (40\%) with 3 still alive 1, 2 and 6 years post-implant.

Of the 109 patients overall, 19 patients have survived 2 years $(17.4 \%), 9$ have lived at least 3 years $(8.3 \%)$, and 3 have lived 5 years $(2.8 \%)$. Eight of these 19 patients $(42.1 \%)$ developed significant hemiparesis apparently associated with radiation white matter injury. We conclude that brachytherapy is of benefit for highly selected patients with both de-novo and recurrent malignant brain tumours and may confer long-term survival in a small subset of patients. 
A6.

\section{The Role for Transfacial Exposure in Treatment of Tumors of the Anterior Skull Base and Clivus}

\author{
M.G. Hamilton, S. Beals, E. Joganic and R. Spetzler \\ (Calgary, Alberta; Phoenix, Arizona)
}

Resection of extensive, deep seated neoplasms involving the anterior skull base and clivus remains a surgical challenge. The anatomic site of these lesions can be used as a guide to classify a logical approach for transfacial exposure. We have defined six levels at which facial osteotomies can be performed to provide excellent exposure for tumor resection. These include exposures by a transfrontal (Level I), transnasal (level I), transfrontal nasal-orbital (Level III), transnasomaxillary (Level IV), transmaxillary (Level V), or transpalatal (Level VI) route. These approaches provide direct access to lesions of the anterior skull base and clivus, thereby minimizing brain retraction. All of these approaches, with the exception of Level IV, can be accomplished without the need for a facial incision. We present our experience with 15 patients who underwent transfacial exposure for resection of extensive anterior skull base or clival neoplasms. Tumor histology included: 5 chordomas; 3 juvenile angiofibromas; and one each of melanoma, chondrosarcoma, fibrosarcoma, malignant fibrous histiocytoma $\mathrm{V}_{2}$ plexiform neurofibro$\mathrm{ma}$, encephalocele, and pituitary adenoma. Surgical exposures utilized for treatment of these 15 patients include 19 levels: 1 each of Level I and Level II; 5 level III; 2 Level IV; 9 Level V; and 1 Level VI. Four patients had multiple levels utilized (Level III and Level V). There was no significant surgical morbidity and no surgical mortality. Eleven of the 15 patients survive. Four patients have died, all as a consequence of tumor progression. We conclude that the transfacial approaches have an important role in the treatment of deep seated lesions of the anterior skull base and clivus.

\section{A7.}

\section{Multidisciplinary Approach to Vascular Skull Base Tumor}

\section{J.-G. Villemure, D. Tampieri, A. Haddad and R. Pokrupa (Montreal, Quebec)}

The base of the skull represents a frontier where neurosurgeons, ENT and head and neck surgeons have to work together in order to accomplish removal of lesions which have both intraand extracranial extension.

In this area, the interventional neurodialogist plays a key role in the pre-operative investigation and treatment of the patient.

We would like to report our experience on the multidisciplinary approach of vascular skull base tumors. The recent advances in imaging allows full assessment of the tumoral size and extension prior to surgery and the possibility to perform 3-D reconstruction helps in the pre-operative evaluation and in the surgical planning.
A8.

\section{Chordoma and Chondrosarcoma: Outcome and Survival Analysis in 29 Patients}

F. Gentili, A. Cabantog, P. Gullane, J. Irish and D. Brown (Toronto, Ontario)

The management of chordomas and chondrosarcomas of the skull base remains disappointing and a major challenge to skull base surgeons. The varied sites of origin, size and extension and their tendency to recur requires careful planning and a variety of surgical approaches. Between 1970 and 1992, 29 patients (18 chordomas, 11 chondrosarcomas) were operated on and the clinical features, radiological studies, operative procedures and survival data analyzed.

Age at presentation ranged from $22-73$ years. Sixty percent were males. The commonest presentation was cranial nerve palsy followed by headache and gait ataxia. Since 1980 a more aggressive surgical approach with combination of craniotomy and transfacial procedures and post-operative radiation (conventional, proton beam) has been the standard therapy. The overall complication rate has been $30 \%$ with no intra-operative or perioperative mortality. In this group only 2 patients have subsequently died of recurrent disease. Prolonged survival, up to 17 years without recurrent disease has been observed although the overall post-operative follow-up period remains short $(5.4$ years).

\section{A9.}

\section{Cerebral Ganglioglioma of Childhood}

J.-P. Farmer, K. Meagher-Villemure, J.L. Montes, A.M. O'Gorman, C.R. Freeman and S. Baruchel (Montreal, Quebec)

We studied 14 children with histologically proven cerebral gangliogliomas. There were 10 males and 4 females. Age at presentation varied from birth to 13 years. 10 lesions were located supratentorially ( 7 temporal), and 4 were in the posterior fossa. Seizure ( 8 cases) was the most common presentation. On CT scan, isodensity ( 9 cases) contrast enhancement ( 9 cases), shift ( 5 cases), cysts ( 5 cases), and calcifications ( 3 cases) were noted. Iso-intensity on T1 (8 cases) and on T2 (8 cases), as well as gadolinium enhancement ( $7 / 8$ cases injected) were common. 7 supratentorial and 2 infratentorial lesions were completely resected. 4 patients were operated more than once. 6 patients underwent adjuvant treatment; 4 of them because the initial diagnosis was different from ganglioglioma Immunohistochemistry and electron microscopy were essential for diagnosis.

There were 2 deaths: 1 related to tumour expansion and the other from acute airway obstruction 3 months post-operatively. Of the remaining 12 patients, 8 patients presenting with seizures are seizure-free, 6 patients are asymptomatic, 4 have mild deficits, and 2 require assistance for activities of daily living. Increased awareness and improved surgical technique makes the prognosis of children with gangliogliomas treated by surgery alone excellent. 


\section{A10.}

\section{Childhood Cerebral Hemispheric Gliomas}

A. Kassam, E.C.G. Ventureyra, M.J. Higgins, D. Keene, E. HSU and P. Humphreys (Ottawa, Ontario)

Childhood Cerebral Hemispheric Gliomas (CHG) represent a unique pathological entity whose natural history and optimal treatment have not yet been clearly defined. The authors report a retrospective review from 1974-1993 covering 200 consecutive pediatric CNS tumors treated at the Children's Hospital of Eastern Ontario. Of these, 196 cases presented adequate tissue for pathological characterization. All tumors were histologically graded my modified Kernohan's criteria. 41 (21\%) patients had confirmed CHG: astrocytoma I-IV, oligodendrogliomas, ependymomas, gangliogliomas, or PNET with clear histological evidence of glial differentiation. Infratentorial, optic, hypothalamic, thalamic and intraventricular tumors were excluded. Patient age ranged from in utero to $16 \mathrm{yrs}$. There was an almost equal incidence of malignant $(56 \%)$ to benign $(44 \%)$ tumors. Overall, males were twice as likely to harbor $\mathrm{CHGs}$ as were females. The most prevalent clinical features at presentation were headache or seizures. The survival of glioblastoma children was similar to that reported for adults: less than one year. However, grade III astrocytoma children showed an unexpectedly protracted survival of 5 yrs. Gross total resection of benign $\mathrm{CHG}$ significantly decreased the recurrence rate from $85 \%$ to $30 \%(\mathrm{p}<0.05)$. Subpial extension (SPE) of neoplastic cells was found to be a powerful indicator of likely recurrence in benign $\mathrm{CHG}$ $(p<0.001)$. These data may significantly alter the management of childhood $\mathrm{CHG}$, but given the relatively small numbers in our study, a multi-centered prospective analysis is warranted to confirm our findings.

\section{B. CEREBROVASCULAR DISEASE}

\section{B1.}

\section{Seizures After Stroke}

C.F. Bladin, P. Johnston, A.V. Alexandrov and J.W. Norris (Toronto, Ontario)

We are studying the frequency, pathogenesis and outcome of seizures after stroke. We evaluated 113 consecutive stroke patients of whom $92 / 1113$ patients $(8.3 \%$ ) had seizures, including $14 / 126(12 \%)$ hemorrhages, and 78/987 (8\%) infarcts $(p=n . s)$; the majority $(65 \%)$ occurred in the first week; a further $25 \%$ occurred 6 to 12 months after stroke.

Large cortical infarcts produced more seizures than subcortical $(\mathrm{p}<0.004)$, but not for hemorrhages. 35/78 (53\%) seizures were either simple partial, or $2^{\circ}$ generalised seizures. Recurrent seizures were more common in patients with hemorrhages (100\%) than infarcts $(41 \%)$ during a mean follow-up of 340 days. The mean lesion volume on $\mathrm{CT}$ did not differentiate seizures from non-seizures for hemorrhages but did for infarcts ( $76 \mathrm{~cm}^{3}$ vs. $47 \mathrm{~cm}^{3} \mathrm{p}<0.02$ ). Comparison of SPECT:CT infarct lesion volumes for seizure patients revealed the SPECT flow lesion to be 2.8 times greater than CT. Cisternal/ventriclar blood did not predict seizures. Seizures were not more common in cadioembolic strokes. Seizure patients had worse morbidity $(p<0.02)$, and mortality $(p<0.05)$. On EEG focal slowing and epileptiform changes were most common in infarct related seizures $(\mathrm{p}<0.0001)$, but not hemorrhages.

Post stroke seizures are common; For infarcts the size of the ischemic penumbra is important; the precise cause in hemorrhages in unclear.

\section{B2.}

\section{Non-Invasive Diagnosis of Carotid Artery Dissection}

\author{
A. Mclean, A.V. Alexandrov, C. Geenen and G. Cheung \\ (Toronto, Ontario)
}

Carotid artery dissection is associated with considerable hemodynamic changes. Magnetic Resonance angiography (MRA) and Transcranial Doppler sonography (TCD) are noninvasive methods to evaluate intra- and extracranial circulation.

Over the past two years 3 cases of symptomatic carotid artery dissection were seen at the Acute Stroke Unit. Patients were 32 56 years old. On admission TCD was performed in the Emergency room setting (TC-EME 2-64). A reversal of the unilateral ophtalmic artery and collateral flow through the anterior communicating artery were detected. The carotid artery dissection was confirmed at the conventional angiography. SPECT showed a decreased perfusion in the unilateral middle cerebral artery. MRA and serial TCD were performed for follow-up. The improvement of the blood flow in the extracranial internal carotid artery (MRA) correlated with normal TCD.

Although rare, carotid artery dissections may have an unusual clinical presentation. TCD may be a useful screening tool and its combination with MRA may be used to monitor the patients' recovery.

B3.

\section{Carotid Angioplasty to Prevent Stroke}

A.V. Alexandrov, G. Cheung, L. LeBrun, J. Raymond, R. Maggisano and J.W. NorRIS (Toronto, Ontario; Montreal, Quebec)

Although carotid endarterectomy is the treatment of choice for high-grade carotid atherosclerosis, it is associated with some risk of perioperative complications. We, therefore, report a preliminary experience with carotid angioplasty at two centres.

Carotid angioplasty was performed on 20 patients (44-78 yrs of age) with $\geq 70 \%$ symptomatic carotid stenosis who refused or were unsuitable for carotid surgery. Of 20 patients, the procedure successfully restored the lumen in 17 . Complications included minor stroke in one and carotid artery rupture in another patient. Immediate and early restenosis occurred in 3 patients. During the follow-up (up to 2 years) there were no significant complications.

Carotid angioplasty may have a place in the management of severe symptomatic carotid stenosis. 
B4.

\section{The Asymptomatic Cervical Bruit Study: Progress Report}

R. Cote and The Asymptomatic Cervical Bruit Study Group (Montreal, Quebec)

Antithrombotic treatment using ASA has not been rigorously tested in asymptomatic carotid disease. The Asymptomatic Cervical Bruit Study was designed to study the natural history of asymptomatic carotid disease and also the efficacy of ASA $(325 \mathrm{mg} /$ day) in preventing ischemic events in patients with high grade stenosis $(\geq 50 \%)$. Six Canadian centres are participating in this prospective clinical trial. Patients are submitted bi-annually to a clinical examination and cervical ultrasonography. Clinical end-points include: stroke, TIA, MI, unstable angina, and death. As of October 1993, a total of 709 patients were enrolled, 363 in the natural history portion and 346 in the controlled trial. Mean age was 65.2 years and average follow-up 33.6 months. Risk factors included: hypertension $56.2 \%$, hyperlipidemia $47.5 \%$, smoking $34.7 \%$, heart disease $27.1 \%$, diabetes $17.5 \%$. At initial visit, about half of the patients had a carotid stenosis of $50 \%$ or greater. Progression of arterial disease was documented in $25 \%$ of patients. The results of this study should permit firmer recommendations in these patients regarding 1) influence of risk factors on outcome 2) efficacy of ASA in reducing ischemic events in higher risk patients.

B5.

\section{Monitoring of the Arterial Stenosis with TCD}

\section{A.V. Alexandrov and M. Gawel (Toronto, Ontario)}

Transcranial Doppler sonography (TCD) is a non-invasive way of detecting the middle cerebral artery (MCA) patency.

We report a case of cerebral vasculitis in a 46-year-old woman. She was a heavy smoker, obese, and was referred to a neurologist as having headaches. Her neurological status was normal. At the initial TCD examination increased blood flow velocities $(\mathrm{BFV})>190 \mathrm{~cm} / \mathrm{sec}$ were detected in both MCAs indicating multiple stenotic lesions. Cerebral angiography confirmed the diagnosis. The patient received a theraphy with steroids. During the course of treatment serial TCD were performed and a decrease in BFVs to the normal values $(<140$ $\mathrm{cm} / \mathrm{sec}$ ) was found over the 6 month period. The repeated cerebral angiogram demonstrated regression of MCA stenoses.

TCD may be a useful adjunct in monitoring patients with intracranial arterial lesions.

B6.

\section{Carotid Stenosis and Peripheral Vascular Disease}

\section{N.A. Alexandrova and R. Maggisano (Toronto, Ontario)}

The Goal of the Study was to assess the incidence and severity of carotid stenosis in patients with peripheral vascular disease (PVD) using color-coded duplex ultrasound (CCDU).

Results: 309 consecutive patients with PVD were studied prospectively. Mean age was $70 \pm 9$ yrs. Two hundred $(65 \%)$ had significant $(\geq 30 \%$ linear $)$ carotid stenosis. Of these $28 \%$ were symptomatic (prior stroke/TIA) and $72 \%$ were asymptomatic. $50 \%$ of symptomatic patients had moderate (30$69 \%$ linear) stenoses. Males had more severe $(\geq 50 \%)$ carotid stenosis compared to females (66 of 97 vs. 39 of $75, p=0.04$ ). More symptomatic patients had hypertension compared to asymptomatic group ( 31 of 55 vs. 57 of $145, p=0.04$ ). More females with carotid stenosis had cardiac disease compared to females without carotid stenosis ( 54 of 86 vs. 14 of $38, p=0.01$ ).

Conclusions: PVD is associated with a high incidence $(65 \%)$ of carotid artery disease in the seventh decade of life. Screening with CCDU is advocated in patients with PVD. Accurate clinical assessment may reveal more symptomatic patients with moderate carotid stenoses required for NASCET.

\section{B7.}

\section{A New Method of Measuring Carotid Stenosis - the Toronto Stenosis Index}

C.F. Bladin, A.V. Alexandrov, J. Murphy, R. Maggisano and J.W. NORRIS (Toronto, Ontario)

Methods of measuring ICA stenosis are those used in the NASCET, ECST trials. Limitations with these linear techniques include poor contrast enhancement of distal ICA, overlying vessels, and difficulties with carotid bulb estimation. We developed a new technique, the Toronto Stenosis Index (TSI), that avoids the problems of distal ICA, or carotid bulb measurements.

The TSI is based on the known fixed anatomical relationship between CCA diameter, and that of the ICA $(1.2 \times$ CCA diam $=$ ICA bulb diam). ICA diameter can be easily estimated from measurement of the CCA, a straight vessel free of atheroma. We evaluated the angiograms of 65 patients (130 vessels) with carotid stenosis measured by NASCET, ECST, TSI, and also duplex. In a subgroup of 30 patients undergoing carotid endarterectomy comparison was made to the carotid plaque. There was considerable intra-method variation (ANOVA) with NASCET $(p<0.001)$, but not for TSI. There was significant difference between NASCET and duplex $(p<0.003)$, but not between TSI and duplex. Comparison of carotid plaque to area derivations of these methods revealed only correlation with TSI and duplex, but not NASCET or ECST. A TSI nomogram was created allowing measurement of linear and area percent stenosis. Doppler and TSI are the only techniques validated for accurate measurement of carotid stenosis.

\section{B8.}

\section{Lipoprotein(a) Levels in Symptomatic Cerebrovascular Disease}

\section{A. Rajput, A. Shuaib and J. KalRa (Saskatoon, Saskatchewan)}

Elevated serum lipoprotein(a) levels have been associated with accelerated atherosclerosis. The relationship between abnormal lipids and stroke is not clear. Our objective was to prospectively evaluate serum lipoprotein(a) levels in patients with symptomatic cerebrovascular disease compared with agematched controls.

53 patients admitted to Clinical Investigative Stroke Unit with diagnosis of either ischemic stroke or transient ischemic 
attack (TIA) and 58 age-matched controls were enrolled between September 1989 and October 1990. Comprehensive lipid profile including lipoprotein(a) levels were obtained 6 weeks after ischemic event. No patients had cranial CT and majority had carotid ultrasound.

Mean serum lipoprotein(a) levels between patients (218.9 \pm $157.4 \mathrm{mg} / \mathrm{L})$ and controls $(157.8 \pm 150.0 \mathrm{mg} / \mathrm{L})$ were significantly different $(\mathrm{p}<0.025)$. Median values for patient or control had history of cardiac disease. All patients and controls were $218 \mathrm{mg} / \mathrm{L}$ and $57 \mathrm{mg} / \mathrm{L}$, respectively. Our study shows significantly higher mean lipoprotein(a) levels in patients with recently completed stroke or TIA compared with age-matched population. Further studies are necessary to better elucidate the relationship between abnormal lipids and cerebrovascular disease.

B9.

\section{D.N.R. Orders in Acute Stroke}

A.V. Alexandrov, C.F. Bladin, L.T. Smurawska and J.W. NORRIS (Toronto, Ontario)

The criteria for ordering "Do-Not-Resuscitate" (DNR) in acute stroke patients have never been clarified.

450 consecutive patients with acute stroke admitted to a University teaching hospital were studied prospectively. The DNR status was ordered in $77 \%$ of patients presenting with severe neurological deficit (C.N.S. $\leq 5.0)$ and $80 \%$ of those died. $55 \%$ of DNR patients were females. $31 \%$ had cerebral hemorrhage and $69 \%$ had ischemic strokes. $53 \%$ of DNR orders were written on the first admission day. Only $8 \%$ of patients were not mentally impaired when DNR status was written in the chart. Physicians had difficulties with families in deciding DNR in $8 \%$ of cases. $47 \%$ of DNR patients had decreased nutrition. If a patient was initially admitted to the Intensive Care Unit (26\%) DNR was rarely ordered.

DNR orders were more likely to be written if an acute stroke patient was more than 60 years old, mentally impaired, admitted to the Stroke Unit or had an intracerebral hemorrhage.

\section{B10.}

Enlargement of the Opposite Pupil as a False Localizing Sign in Intraperenchymal Frontal Hemorrhage.

R. Chen, R. Sahjpaul, R. Del Maestro, L. Assis and B. YOUNG (London, Ontario)

Ipsilateral third nerve palsy with early pupillary enlargement is an important sign of transtentorial herniation from a supratentorial mass lesion. We report a case of frontal, intraparencymal hemorrhage in which the first ocular manifestation of transtentorial herniation was enlargement of the contralateral pupil. The ipsilateral pupil dilated only after complete oculomotor palsy of the contralateral eye. Following partial frontal lobectomy and removal of blood clot, the ipsilateral third nerve recovered before the contralateral third nerve. Clinical observations localized the contralateral third nerve lesion to an extra-axial site. The inferior frontal location of the hematoma is likely important in producing the force vectors involved to cause contralateral third nerve palsy and the possible mechanisms of contralateral third nerve compression will be discussed. To our knowledge, this is the first example of pupillary enlargement as a false localizing sign from a contralateral, supratentorial, intraparencymal mass lesion. Its recognition can be helpful in reconciling conflicting neurological signs and in the localization of lesions. Such information is often crucial in the interpretation of neuroradiological investigations and in patient management.

B11.

\section{Intracerebral Haematoma Following Aneurysmal Bleed}

D. Sirhan, M. Bojanowski and N. Gauthier (Montreal, Quebec)

We studied 200 consecutive patients surgically treated for ruptured cerebral aneurysm, amongst which 58 (29\%) had associated intracerebral haematoma. Early surgery and ICP monitoring were combined with hypervolemia, hemodilution and administration of nimodipine. Patients presenting with haematoma were more liable to harbour aneurysms of the anterior communicating (AcomA) and middle cerebral (MCA) arteries. They were also more likely to have had a previous subarachnoid bleed (48\% vs. $16 \%)$. Clinical grade was generally worse on admission and amongst those presenting with poorer grades, AcomA aneurysms were associated with a worse prognosis than MCA aneurysms. This may be due to concomitant intraventricular haemorrhage (IVH). Following treatment, $43 \%$ of grade IV Hunt and Hess patients with haematoma had a good prognosis (60\% once IVH patients were excluded). These results indicate that patients with intracerebral haematoma often have prior unnoticed bleeding episodes. Moreover, presence of haematoma correlates with poor clinical presentation but with our present approach, clinical outcome is mostly influenced by aneurysm location and associated IVH.

\section{NEUROMUSCULAR DISEASE}

\section{C1.}

Cardiovascular Autonomic Testing: Are Multiple Tests of Heart Rate and Blood Pressure Redundant?

\section{J.D. Stewart and J. Bodkin (Montreal, Quebec)}

Objective: To determine if there are correlations (hence redundancies) between different tests of heart rate $(\Delta \mathrm{HR})$ and systolic blood pressure $(\triangle \mathrm{SBP})$ changes.

Background: Batteries of cardiovascular autonomic tests that include several measures of $\triangle \mathrm{HR}$ and $\triangle \mathrm{SBP}$ are widely used. If these measures correlate, a common physiological mechanism is likely and it would be redundant to perform all measures.

Design/Methods: in 40 healthy women 20-40 years, beat-beat BP (Finapres) and HR were recorded during 3 perturbations deep breathing (DB), lying-standing (LS) and Valsalva (VM). $\triangle \mathrm{HR}$ was measured in 3 ways from HR, 4 from LS, 5 from VM; $\triangle \mathrm{SBP}$ in 5 ways from LS, 9 from VM. Pearson product moment correlations were performed between the $\triangle \mathrm{HR}$ measures, and between the $\triangle \mathrm{SBP}$ measures.

Results: 1) $\Delta \mathrm{HR}$ measures, and $\triangle \mathrm{SBP}$ measures respectively, correlated highly within each perturbation. 2) $\Delta \mathrm{HR}$ measures 
from the 3 perturbations correlated poorly with each other. 3) $\triangle$ SBP measures from LS and VM correlated poorly.

Conclusions: These low correlations between $\triangle H R$ and between $\triangle \mathrm{SBP}$ measures in the 3 perturbations suggest differing underlying physiological mechanisms and supports the use of measures derived from different perturbations in an autonomic test battery.

C2.

Dissociated Wasting of the Medial and Lateral Hand Muscles with Motor Neuron Disease

\section{A.J. Wilbourn And P.J. SweEney (Cleveland, Ohio)}

We recorded compound muscle action potentials (CMAP) from three hand sites - median, thenar; ulnar, hypothenar; ulnar, first dorsal interosseous (FDI) - in patients with motor neuron disease (MDN). In 50 hands (of 39 patients), one or more CMAP amplitudes were abnormal; overall, 120 of the 150 responses were very low/unelicitable. Three patterns occurred: only 1 of 3 responses was low - hands (4 FDI; 2 thenar); 2 of 3 responses were abnormal - 18 hands (16 FDI/thenar; 2 FDI/hypothenar); all 3 responses were abnormal -26 hands (with the lateral hand muscles more severely affected in 13). The FDI CMAP was substantially lower than the hypothenar CMAP in 33 of 50 hands; the reverse was never seen. Also, thenar changes usually were similar to FDI, rather than hypothenar, changes.

Conclusion: In the cervical spinal cord: (1) the anterior horn cells supplying the FDI muscles are more closely aligned to those supplying the median-innervated thenar muscles than to those supplying the ulnar-innervated thenar muscles; (2) the neuron pool innervating the lateral hand muscles often is affected earlier, and more severely, with MND than the group innervating the medial hand muscles.

C3.

Motor Neuron Disease Presenting with Acute Respiratory Failure: Clinical, Electrophysiological and Pathological Studies in Four Cases

R. Chen, H. Remtulla, F. Grand'Maison, B. Toth, D.A. RAMSAY and C.F. BolTon (London, Ontario; Sherbrooke, Quebec)

Respiratory failure is a major cause of mortality in motor neuron disease (MND). It usually occurs late in the course of the disease and is rarely a presenting symptom.

We studied the clinical, electrophysiological and pathological features of four patients with MND who presented with acute respiratory failure requiring intubation and mechanical ventilation. These were two male and two female, aged 55 to 78 . The neurological cause of respiratory failure was considered only after investigations for cardiac or pulmonary causes were negative. Although they were all previously undiagnosed, dyspnea or limb weakness were present for several weeks to months prior to presentation. The clinical examination in all cases revealed variable degrees of muscle atrophy, fasciculation and weakness. Phrenic nerve stimulation invariably showed severely reduced or absent diaphragmatic compound motor action potential. Needle electromyography demonstrated widespread denervation, including the diaphragm. Autopsy confirmed severe involvement of the phrenic nerve nucleus.

MND can therefore present as acute respiratory failure secondary to diaphragmatic paralysis. Electrophysiological studies are invaluable in demonstrating early and severe involvement of the respiratory system and in providing a guide to prognosis which is crucial in subsequent management decisions.

\section{C4.}

\section{Ulnar Neuropathies in the Hand Caused by Canes, Crutches, and Walkers}

\section{A.S. Wilbourn and P.J. SweEney (Cleveland, Ohio)}

We report six patients ( 3 men, 3 women) who developed a total of 9 ulnar neuropathies in the hand (UN-H) -5 right, 4 left - while using canes, crutches, or walkers, (often for only short periods of time) for various lower extremity disorders. Three of the 6 patients had unilateral lesions ( 2 from cane use, 1 from crutches), while 3 had bilateral lesions ( 1 from crutch use, 2 from walkers). Clinically, in the 6 patients there were sensory complaints in an ulnar hand distribution in 3 ; motor hand symptoms in 1; sensory and motor symptoms in 1; the final patient was asymptomatic (referred for co-existing carpal tunnel syndrome). Electrodiagnostically, the ulnar nerve conduction studies were abnormal (decreased amplitudes more often than prolonged latencies) in all hands, 7 of 9 sensory and 6 of 9 motor; needle electrode examination showed fibrillation potentials in ulnar-innervated hand muscles in 5 of the 9 patients.

Conclusions: UN-H can develop, unilaterally or bilaterally, when canes, crutches, or walkers are used, even for brief time periods. An unusual feature of these $\mathrm{UN}-\mathrm{H}$ is that the ulnar sensory fibers frequently are affected, in contrast to most other etiologies, which typically involve only motor fibers.

\section{C5.}

\section{Adjuvant Breast Disease in Patients with Saline Filled Silicone Elastmer Shell Implants}

\section{B. OStermeyer ShoAib and B.M. PATten (Houston, Texas)}

Increasing concern has been expressed about the safety and efficacy of saline filled silicone elastomer shell breast implants because they have been associated with systemic diseases, too. We report 10 women with saline breast implants who developed a peripheral neuropathy syndrome $(n=9)$ or motor neuron disease syndrome $(n=1)$ at a median latency period of 6.4 years (range $0-13$ years) after implant surgery. The patient with motor neuron disease syndrome died recently. Three of the patients with neuropathy had evidence of systemic vasculitis. Raynaud's phenomena was seen in $8 / 10$ and vasospasm of major veins of lower extremities on phlebography was seen in $1 / 10$. Autodirected antibodies were found in $9 / 10$. Sural nerve biopsy showed loss of myelinated fibers in $4 / 5$ and vasculitis in $1 / 5$. Biceps muscle biopsy showed type II atrophy in $2 / 6$, neurogenic atrophy in $1 / 6$, myositis in $1 / 6$, fasciitis in $1 / 6$ and vasculitis in 1/6. Implants were removed in 8 patients and $4 / 8$ had ruptured 
implants. Implant capsule biopsy showed foreign body giant cells and free silicone in $3 / 5$ and foamy histiocytes in $2 / 5$. Pectoralis muscle biopsy showed neurogenic atrophy in $3 / 5$, myositis in $2 / 5$ and fasciitis and free silicone in $1 / 5$. Saline breast implants can cause a systemic autoimmune disease. Therefore, further investigations about this kind of implant are necessary.

C6.

\section{Adjuvant Breast Disease: Patients with Atypical Systemic Disease and Implant Failure}

\section{B. Ostermeyer Shoaib, B.M. Patten and D.S. Calkins (Houston, Texas)}

We evaluated about 1500 women who developed "Adjuvant Breast Disease" after breast implant surgery or silicone injections into breasts or face and report here the results of the first 100 women. Symptoms occurred at a median latency period of 6 years (range 0-24 years) after breast implant surgery $(n=97)$ or silicone fluid injection into breasts $(\mathrm{n}=3)$. Commonest symptoms were weakness $(95 \%)$, fatiguability $(95 \%)$, myalgia $(90 \%)$, morning stiffness $(89 \%)$, arthralgia $(81 \%)$, memory loss $(81 \%)$, sensory loss $(77 \%)$, headache $(73 \%)$ and dry eyes and dry mouth (72\%). Five patients died during the study. Abnormal levels of serum immunoglobulins or complement were found in $57 \%$ and autoantibodies in $78 \%$. Sural nerve biopsy was abnormal in $80 \%$ (loss of myelinated fibers in $79 \%$ ). Biceps muscle biopsy was abnormal in $58 \%$ (neurogenic atrophy in $27 \%$ ). Ninety-six patients underwent implant removal and $60 \%$ were found to have ruptured implants. A Kaplan-Meier implant survival analysis was plotted and showed that all types of implants tend to rupture, if not removed before that. At time of removal, a pectoralis major muscle biopsy was taken which was abnormal in $89 \%$ (neurogenic atrophy in 55\%). Biopsy of the implant capsule was abnormal in $94 \%$. Silicone Breast Implants and silicone fluid injections can cause a systemic autoimmune disesase probably due to a global activation of the immune system. The disease characteristics and laboratory features differ from idiopathic diseases.

C7.

Use of Intrathecal Morphine for Long Term Management of Intractable Reflex Sympathetic Dystrophy

\author{
W.J. Becker, C.J. Harris, D.P. Ablett and O.N. Dold \\ (Calgary, Alberta)
}

Two patients with intractable life-threatening reflex sympathetic dystrophy (RSD) involving the lower limb have been treated at our hospital with intrathecal morphine using Infusaid Model 400 implanted pumps. Both patients did not obtain lasting relief from conventional medical therapy or surgical sympathectomy. Both responded well to intrathecal morphine, and remain on this therapy over five years after initiation of intrathecal therapy. One patient was able to return to full-time work; the other is a homemaker who is able to drive her car, shop, and independently look after her home.
Both patients experienced exacerbations of their RSD secondary to subsequent trauma of the involved limb which responded to increases in the intrathecal morphine dose. One patient suffered a severe exacerbation secondary to interruption of morphine delivery from catheter rupture, which resolved with catheter repair. The first sign of catheter breakage was an erythematous rash over the pump site. Intrathecal morphine dosage over the years varied from 8 to $110 \mathrm{mg}$ per day. No pump or catheter infections occurred.

Intrathecal morphine delivered via an implanted pump is an important therapeutic option for patients with severe RSD who are unresponsive to other treatment modalities.

\section{C8.}

\section{Duchenne Muscular Dystrophy: The Measurement of Quality of Life}

D.L. Fehlings, P. Rosenbaum, G. Canny, K. Simpson, N. LADEK and P. WEBSTER (Toronto; Hamilton, Ontario)

The clinical management of Duchenne Muscular Dystrophy (DMD) has recently become increasingly complex. With the technological development of non-invasive long-term ventilation it is now feasible to significantly lengthen the life of an individual with DMD. This has sparked considerable controversy amongst health professionals who express concerns over the prolongation of suffering and a poor quality of life (QL). To resolve this controversy information on QL is required.

The primary objective of this research was to develop a disease specific health status measure that would accurately assess QL in individuals with DMD and hypoventilation. The methodology for developing the questionnaire and results will be presented. Seventy-six items were generated for potential use in the questionnaire by a literature review, interviewing 10 clinical experts and 20 individuals with DMD and hypoventilation. This number was then reduced to 24 by selecting items with the highest frequency-importance product. Test-retest reliability was established with a low coefficient of variation $(0.11)$ reflecting low within subject variation when the subject remained stable. Construct validity was established with correlations of QL measure to the individuals global rating of $\mathrm{QL}(\mathrm{r}=0.61)$, to the Sickness Impact Profile $(r=0.53)$, and to forced vital capacity $(\mathrm{r}=-0.43)$. The clinical applications of nasal ventilation, and ethical principles guiding decision making will also be discussed.

\section{C9.}

\section{Prominent Axial Muscle Weakness as a Presenting Feature in Mitochondrial Myopathy}

A.L. Genge, D. Arnold, E. Shoubridge, S. Carpenter and G. KARPATI (Montreal, Quebec)

Prominent muscle weakness and/or fatiguability of neck extensor and trunk erector muscles are relatively rare and most often observed in myasthenia gravis and amyotrophic lateral sclerosis. Here we report 4 elderly patients who presented with conspicuous head-drop and/or severely stooped posture due to weakness of trunk erector muscles. The patients $(70 / \mathrm{M}, 73 / \mathrm{F}$, $82 / \mathrm{M}, 85 / \mathrm{F}$ ) also showed relatively minor proximal limb muscle 
weakness and fatiguability. EMG showed spontaneous activity in the paraspinal muscles. Serum CK activity was 167-205. Tensilon tests and single fiber EMG showed no neuromuscular transmission defect. Muscle biopsy from deltoid showed a significant number of ragged red fibers (RRF) and equivalents which were negative for cytochrome oxidase. mtDNA analysis showed multiple small deletions.

This disease has been called old folks' mitochondrial myopathy, which is presumed to be caused by cumulative effects of deleted mtDNA in mitochondrial oxidative phosphorylation. These deletions are presumed to accumulate in muscles of old patients.

This is a very inconvenient and even debilitating disease. Currently, it can only be treated by supportive measures.

\section{PEDIATRIC NEUROLOGY}

(General and Epilepsy)

\section{D1.}

\section{Lennox-Gastaut Syndrome associated with Brain Tumor}

J. Hukin, M.B. Connolly, J. Kestle and K. Farrell (Vancouver, British Columbia)

Lennox-Gastaut syndrome is associated with a poor prognosis both for seizure control and neurological outcome. The etiology is often unknown. However, brain tumors have been described as a rare cause.

The patient was a nine-year-old boy who developed complex partial seizures at three months of age. These were refractory to medical treatment and he developed tonic, myoclonic and atonic seizures by two years of age. The interictal EEGs demonstrated abnormal background, generalized slow pike-waves and a left temporal focus. The seizures were intractable and he developed mental retardation. Two CT scans of the brain at 3 months and at three years and an MR scan at three years were normal. A mild right hemiparesis developed and at 9 years of age an MR head scan demonstrated increased signal in the left temporal lobe. Following resection of a left temporal lobe low grade astrocytoma he became seizure free with slight improvement in cognitive function.

In this patient, there is an unusual association of LennoxGastaut syndrome with brain tumour. The occurrence of partial seizures as the initial seizure type, the focal left temporal EEG abnormalities, and remission of seizures following excision of the tumor suggest a causal relationship.

\section{D2.}

\section{Congenital CNS Tumors: Report of 6 Cases}

K. Meagher-Villemure, J.L. Montes, J.P. Farmer and A.M. O'GORMAN (Montreal, Quebec)

Intracranial tumors developing in utero or with presenting symptoms within the first 60 days after birth are extremely rare. We are describing the Montreal Children's Hospital experience of the past ten years. Seven congenital central nervous system tumors over 160 tumors were found. Six babies presented with hydrocephalus, three patients had their tumor located in the brainstem, two in the cerebellum and one in the pineal region, and one in the spinal cord. Histologically, astrocytoma, PNET and ganglioglioma were found in the brainstem, a rhabdoid tumor was involving the whole length of the spinal cord, one metastasis from a Wilm's tumor was found in the cerebellum, one cerebellar medullo-epithelioma, and one pinealoblastoma of the pineal region. The prognosis of these tumors is poor for undifferentiated and infiltrative types and good for those welldifferentiated and resectable. The pathogenesis of these tumors is discussed in regard to the genetic factors, familial occurrence, intrauterine infections and environmental factors.

\section{D3.}

\section{Familial Febrile Convulsions and Temporal Lobe Epilepsy}

J. MAHER and R.S. MCLACHLAN (London, Ontario)

Background. As part of a molecular genetics study of febrile convulsions, factors associated with the subsequent development of temporal lobe epilepsy and possible modes of transmission of febrile convulsions were investigated. Methods. Seven large families with familial febrile convulsions were assessed. Detailed questionnaires were compiled on all affected patients. The diagnosis of temporal lobe epilepsy was based on seizure history and electroencephalography. Results. Fifty-three of 60 family members, each of whom had febrile convulsions, could be analyzed. Of these, 41 had no further seizures (Group I), 9 developed temporal lobe epilepsy (Group II), and 3 had other types of epilepsy. Temporal lobectomies in 4 patients revealed mesial temporal sclerosis. Mean duration of the longest febrile convulsion Group I was $10 \mathrm{~min}$. compared to $112 \mathrm{~min}$. in Group II (Mann-Whitney $U$ test, $p<0.005$ ). The total number, the maximum number in any one day, and the age at onset of febrile convulsions did not differ significantly between patients who did or did not develop temporal lobe epilepsy. A focal neurological deficit occurred in only one patient (Group II). Inheritance of febrile convulsions in these families is most like polygenic, although an autosomal dominant mode of transmission with reduced penetrance is a possible alternative. Conclusions. Duration of febrile convulsions is the most important determinant of progression to temporal lobe epilepsy in patients with familial febrile convulsions. (Supported by the Savoy Foundation.)

\section{D4.}

\section{Specificity of IHS Criteria in Childhood Headache}

\section{S. SESHIA and J. Wolstein (Winnipeg, Manitoba)}

We have previously suggested that the International Headache Society (IHS) rules and criteria (1988) can be applied to the majority of children referred to a Pediatric Neurology Clinic. We now report on a prospective study designed to assess the relative specificity of the criteria.

The 30 children in this study, aged 4 years to 15 years, were seen in 1993. The study forms allowed more than one headache type to be diagnosed by IHS criteria in the same child. General Rule 7 in the IHS document was disregarded because of its arbitrary and self-fulfilling nature. 
Six children had a single headache type. In 19 children headache, characteristics in the same episode fulfilled IHS criteria for both a migraine and a tension-type headache. The headaches would have been misclassified in 5 children, one a child with shunt malfunction whose headache features met IHS requirements for migraine without aura and a tension-type.

We support the philosophy behind the IHS document. However, revisions are necessary to minimize misclassification and enhance general application. These include, (i) exclusion of arbitrary rules, (ii) greater emphasis on "organic" causes and (iii) re-appraisal of the criteria that distinguish between migraine (types $1.1 \& 1.7$ ) and tension-type headache.

\section{D5.}

Electrophysiological Studies in the Paediatric Critical Care Unit

M. McKeown, R. Chen, H. Remtulla, L. Bernardi, B. Toth, J. GiLletT, S. LeVIN and C. Bolton (London, Ontario)

We present our experience of electrophysiological studies of 15 patients (age 7 days to 16 years) in the paediatric critical care unit (PCCU), conducted over a period of 2.5 years.

Limb weakness and respiratory difficulties were the most common reasons for referral. Standard motor and sensory nerve conduction studies, including $\mathrm{F}$ waves and $\mathrm{H}$ reflexes, needle electromyography of limb muscles and neuromuscular junction transmission studies, were conducted with no difficulty or complication. Assessment of the autonomic nervous system with cardiac R-R interval variation and sympathetic skin response, somatosensory evoked potentials, phrenic nerve conduction studies and needle electromyography of the diaphragm were performed in selected patients.

The diagnoses demonstrated included lack of central drive as a cause of respiratory failure, neuropathies such as GuillainBarré syndrome, critical illness polyneuropathy, localized brachial plexus injuries, neuromuscular transmission defects and myopathies. Repeat studies in six patients helped in following the course of the disease and confirming the diagnosis. Phrenic nerve conduction studies and electromyography of the diaphragm were useful in establishing the cause for respiratory failure.

We conclude that comprehensive electrophysiological studies can be performed in the PCCU. They are often useful in establishing the diagnosis and provide a guide to prognosis.

\section{D6.}

Therapeutic Electrical Stimulation (TES) in Children with Motor Disorders: a Phase 1/Phase 2 Study

\section{P. Steinbok, A. Reiner and J. Kestle (Vancouver, British Columbia)}

Therapeutic electrical stimulation (TES), which involves low intensity electrical stimulation applied for many hours daily during sleep, has been introduced recently, with promising results reported in a number of motor disorders.

We describe a phase 1/phase 2 study to explore the feasibility and effectiveness of TES performed for 12 months in three groups of children: 1) Five children more than two years after selective dorsal rhizotomy for spastic diplegia, 2) Five children with hemiplegia and a spastic hand, and 3) Five children with meningomyelocele and quadriceps function.

TES was simple to perform and was well tolerated by children and their caregivers. The results suggest that TES may be beneficial for spastic diplegics after selective dorsal rhizotomy, by increasing strength, decreasing spasticity and improving motor function as assessed by the Gross Motor Function Measure (GMFM). For the spastic hemiplegic children, TES tended to increase strength and decrease spasticity in the upper limbs, but had little effect on functional abilities as assessed by either the GMFM or measure of upper extremity skills. In the myelomeningocele population, increased lower limb strength was observed after TES, and in two of three children there was an improvement in bladder and/or bowel function.

\section{D7.}

\section{Relationship of Intraoperative Electrophysiologic Criteria to Outcome After Selective Functional Posterior Rhizotomy}

P. Steinbok, B. Gustavsson, J. Kestle, A. Reiner and D. Cochrane (Vancouver, British Columbia; Stockholm, Sweden)

In our institution the criteria used in selective dorsal rhizotomy (SDR) evolved in three phases. In phase 1 the electrophysiologic criteria of abnormality included low threshold to single stimulus, sustained response to $50 \mathrm{~Hz}$ stimulation, and spread outside the segmental level being stimulated. In phase 2 the electrophysiologic criteria were unchanged, but less of L3/L4 were cut. In phase 3 , the only electrophysiologic criterion used was contralateral and suprasegmental spread. This study examined the relationship between the electrophysiologic criteria and outcome.

77 consecutive children with SDR and a minimum follow-up of 1 year were reviewed, comprising 25,19 and 32 in phases 1 , 2 and 3 respectively. Outcome parameters included quantitative assessments of lower limb spasticity and range of movement, and qualitative assessments of lower limb function. In phase 3, $50 \%$ of the roots were cut, compared to $65 \%$ and $64 \%$ respectively in phases 1 and 2 . In each phase, lower limb spasticity decreased and range of movement increased, with spasticity decreasing least in phase 3 . Over $90 \%$ of children in each phase showed improved lower limb function, but improvement in the level of ambulation occurred in $60 \%, 58 \%$ and $42 \%$ in phase 1,2 and 3 respectively.

\section{D8.}

\section{Canadian Pediatric Ischemic Stroke Registry}

G.A. Deveber and Canadian Pediatric Ischemic Stoke Study GROUP (Hamilton, Ontario)

The epidemiology of ischemic stroke in childhood is uncertain. We initiated a National Registry in January 1992 with participation from all tertiary care pediatric centres $(\mathrm{N}=16)$. As of Nov, $1 / 93$ 136 consecutive children $<18$ yrs ( 96 with complete information) with arterial ischemic stroke or cerebral sinovenous thrombosis were identified. The incidence was 1.05 per 100,000 children 
per yr and was highest in infancy; $<1$ yr $26 \%$; 1-6 yrs: $36.5 \%$; $\geq$ 6 yrs: $37.5 \%$. Vessels involved were large vessel arterial $(42.7 \%)$, small vessel arterial $(36.5 \%)$ and sinovenous $(20.8 \%)$. Etiologies were: unknown $(26 \%)$, cardiac disease $(20.8 \%)$, infection/inflammation (16.7\%), coagulopathy $(7.3 \%)$, dissection $(6.3 \%)$, Moya-moya (3.1\%) and other known (19.8\%). Known outcome at discharge was: $5(9.3 \%)$ dead (of underlying disease), $31(57.4 \%)$ with neurologic sequelae and $18(33.3 \%)$ normal. Anticoagulants were used in $22(22.9 \%)$. The poor outcome supports the need for itervention trials in pediatric ischemic stroke. (Funded by CMH Foundation.)

D9.

\section{The Natural History of Pediatric Familial Cavernous Malformations}

M.G. Hamilton, K. Greene, H. Rekate and J. Zabramski (Calgary, Alberta; Phoenix, Arizona)

Cavernous malformations are vascular abnormalities affecting about $0.5 \%$ of the population occurring in either a sporadic form or a familial form with an autosomal dominant mode of inheritance. We report 17 pediatric patients with familial cavernous malformations. There were 8 boys and 9 girls with a mean age of $12.8 \pm 3.1$ years (range $8-17$ years). The clinical presentation was seizures in one patient (5.9\%), neurological deficit in 2 patients $(11.8 \%)$, and headache in 4 patients $(23.5 \%)$. The remaining patients were identified during screening for a familial cavernous malformation protocol. The mean number of lesions per patient was $4.4 \pm 2.5$ (range 1-11). Three patients (17.6\%) underwent surgical removal of cavernous malformations. The mean duration of followup has been $40.2 \pm 12.7$ months. Seizures developed in 2 patients $(11.8 \%$ ), hemorrhage in 2 patients $(11.8 \%)$, increase in cavernous malformation size in 2 patients and 6 patients $(35.3 \%)$ developed new lesions. The results of this review demonstrate that the familial form of cavernous malformations is a dynamic disease. Serial MR imaging revealed hemorrhage, changes in the number and size, and demonstrated the de novo development of new lesions. These findings suggest that patients with familial cavernous malformations require careful long term followup. MR imaging should be repeated for any significant change in neurologic symptoms. Surgery should be considered, but restricted, for those anatomically significant lesions that produce repetitive or progressive symptoms, or demonstrate evidence of repetitive hemorrhage. The prophylactic resection of asymptomatic lesions does not appear to be otherwise warranted.

\section{D10.}

Pediatric Cerebral Sinovenous Thrombosis - Are Heparin Trials Justified?

\section{G.A. Deveber and M. Andrew (Hamilton, Ontario)}

Cerebral sinovenous thrombosis (SVT) in adults has a significant mortality and morbidity; a recent randomized control trial has confirmed the efficacy and safety of anticoagulation. In children with SVT, it is uncertain whether anticoagulant therapy (AC) is beneficial or justifiable. To obtain preliminary data we performed an analysis of pediatric SVT reported since 1980 in the English literature to evaluate outcome. Criteria were age $\leq$ 18 yrs, radiographic, surgical or post-mortem confirmation of SVT and information on clinical outcome. Of 331 children reported, 161 fulfilled our criteria for inclusion, 15 treated with ACs and 146 treated with no ACs. In the AC treated group 2 of 15 died (both due to their underlying illness) and none had neurological sequelae. In the no-AC group 18 (12.3\%) died and 37 (28.9\%) of the survivors had neurologic sequelae. In conclusion, in children with SVT mortality and morbidity are significant and an intervention trial assessing the potential benefit of AC therapy is indicated.

\section{D11.}

Reversible Axonal Polyneuropathy in Children with Severe Asthma: A Variant of Critical Illness Neuropathy?

A.Y. Manzur, M. Bennett, R.D. Keyes and E.H. Roland (Vancouver, British Columbia)

Critical illness polyneuropathy has been reported in adults with sepsis and multiple organ failure and may relate to nutritional factors, inhalational anesthetics, vecuronium or steroids. We describe transient axonal polyneuropathy in 3 children who were ventilated for status asthmaticus.

Three patients (2, 3 and 7 years) who had normal development and no family history of neuromuscular disease required ventilation for status asthmaticus (duration 9-11 days). All received aminophylline, ventolin, steroids, inhalational anesthesia and vecuronium. Complications included pneumothorax ( 1 patient), elevated liver enzymes (1). After extubation, all children were irritable and had severe symmetrical limb weakness, poor head control and inability to sit unsupported. One child had bilateral facial weakness. Sensory testing was normal. All children recovered completely (2-5 months).

Electrolytes and CPK were normal. Motor and sensory nerve conduction velocities were normal. Amplitudes of compound muscle action potentials were diminished in facial and limb muscles. One patient had mildly abnormal EEG with dysrhythmic background activity.

Severe reversible axonal polyneuropathy in children following ventilation for acute asthma has not been described previously. This unusual complication may be a variant of critical illness neuropathy.

\section{E. EPILEPSY SURGERY}

E1.

\section{Surgery of Temporal Lobe Epilepsy: Results of Various} Modalities of Resections

A. Olivier, X. Palomino-Torres and F. Andermann (Montreal, Quebec; Mexico City, Mexico)

Rationale: The aim of this study is to compare the effect of various modalities of temporal resections on the seizure tendency and to clarify their indications, advantages and limitations. 
Methods: A series of 432 patients operated by the same surgeon $(\mathrm{AO})$ was sub-divided into 3 groups of resections i.e., cortico-amygdalectomy (CA) (77 pts), cortico-amygdalohippocampectomy (CAH) (312 pts) and selective amygdalo-hippocampectomy (SAH) (43 pts) with an average follow-up of $10.1,6.4$ and 2.2 respectively (minimum of 2 years for $\mathrm{CA}$ and $\mathrm{CAH}$ and 1.5 years for $\mathrm{SAH}$ ).

Results: Using Engel's classification, $48 \%$ of CA were in the success group (Class I to III) and $52 \%$ were in the (relative) failure group (Class IV). $89 \%$ of $\mathrm{CAH}$ were in the success group and $11 \%$ were in the failure group. $89 \%$ of $\mathrm{SAH}$ were in the success group, $4 \%$ had significant improvement and $7 \%$ were in the failure group. The aura, when present (approximatively $80 \%$ of cases) disappeared in $19 \%$ of $\mathrm{CA}, 64 \%$ of $\mathrm{CAH}$ and $83 \%$ ofSAH. In reply to a questionnaire answered by these 432 patients, $94 \%$ said they had benefitted from surgery. Memory was felt to be subjectively the same or better in $63 \%$ of $\mathrm{CA}, 84 \%$ of $\mathrm{CAH}$ and $96 \%$ of SAH. It was felt to be worse in $37 \%$ of CA, $16 \%$ of $\mathrm{CAH}$ and $4 \%$ of SAH.

\section{Conclusion:}

1. seizure control is better when the hippocampal formation is included in the resection.

2. the extent of the hippocampal resection is important for better seizure control. Over $85 \%$ of patients having undergone an hippocampal resection think that their memory is the same or better.

3. Cortico-amygdalectomy is associated with a high failure rate $(52 \%)$ on seizure tendency.

E2.

\section{Surgical Treatment of Epilepsy and Low Grade Gliomas of the Temporal Lobe}

W.B. Woodhurst, D.B. MacDonald, J. Wada and K. Berry (Vancouver, British Columbia)

Eighteen patients (11M 7F) had operations for intractable epilepsy associated with low grade glioma of the temporal lobe 1988-1993. Seventeen lesions were gangliogliomas, 1 astrocytoma.

Sixteen lesions were excised ( 3 incomplete) guided by electrocorticography $(\mathrm{ECOG}) .1$ lesion was partially excised and cyst drained. I lesion-epileptogenic tissue excised post resection of tumor. Operations were done under local anaesthesia in six patients.

Four language deficits were exacerbated by surgery three temporarily one permanently mildly. Two patients had reduced verbal memory post-operatively both mildly.

Preop interictal EEG findings (18 pt): 9 unilateral localizing epileptiform. 1 localizing nonspecific. 6 bitemporal independent epileptiform and 1 diffuse abnormality. 1 EEG was normal.

Ictal EEG findings (16 pt): 14 localizing ( 1 invasive) 1 poorly localizing. 1 unlocalized.

ECoG findings (17 pt): 11 localizing-anatomically congruent. 6 less useful correlations.

Outcome (Engel) Class I - 14. Class II - 1. Class III - 3. Class IV - 0. Poor outcome (Class II \& III) correlates with residual tumor (1/3) and residual spikes on $\mathrm{EGoG}(3)$ though neither finding was absolutely predictive.

The issues of resection volume vs. lesion volume/location and ECoG abnormalities will be discussed.
E3.

\section{Epilepsy and Gangliogliomas: Imaging Features}

W.B. Woodhurst, R.N. Nugent and K. Berry (Vancouver, British Columbia)

The CT and MRI characteristics of gangliogliomas in 15 patients with intractable epilepsy were studied.

The tumor was located in the temporal lobe in 14 patients and in the frontal lobe in 1 patient. Temporal lobe tumors were lateral in 5 patients and mesial in 9.

The tumors were relatively small, with 10 measuring 1 to 3 $\mathrm{cm}$. in diameter and only one greater than $5 \mathrm{~cm}$. Mass effect was absent in 5 patients and mild in the other 10; only 2 had evidence of peritumoral edema.

Cystic appearing areas were present in 5 patients; calcification was identified in 6 . Nine patients received intravenous contrast, with 4 demonstrating mild enhancement and 1 having prominent enhancement. Both homogeneous (3) and irregular (1) patterns of enhancement were identified.

Specific MRI characteristics were decreased $\mathrm{Tl}$ intensity in 10 of 13 patients having MRI studies, with increased proton density and $\mathrm{T} 2$ signal in all 13 .

The gangliogliomas in this series present a broad spectrum of CT and MRI features. Cystic change with calcification is relatively characteristic but was found in a minority of patients. The imaging characteristics of these tumors with the differential diagnosis will be discussed.

\section{E4.}

\section{Peri-Insular Hemispherotomy for Seizures}

J.-G. Villemure, J. Montes, J.P. Farmer, C. Mascott and C. MercIER (Montreal, Quebec)

Peri-insular hemispherotomy is the surgical procedure allowing complete disconnection of the hemisphere with minimal brain removal. Only the amygdala and opercular cortex need to be excised; the hemisphere disconnection is then carried from within the ventricle.

Eleven of the last 15 functional hemispherectomies were done using the peri-insular hemispherotomy technique variation. There were 4 males and 7 females. Age ranged from 2 to 32 years. Etiologies for the seizures were hemimegalencephaly in 3 cases, infantile hemiplegia in 4, chronic encephalitis in 2, meningitis in 1 and cardiac embolus in 1. Follow-up ranged from .3 to 3 years. One patient developed early hydrocephalus post-operatively; 9 of the 11 patients have been seizure-free since discharged from the hospital; 2 patients continue to have seizures but at a frequency of $5 \%$ of the original seizure frequency.

Peri-insular hemispherotomy, a variation of functional hemispherectomy appears to be the hemispherectomy technique where the ratio disconnection / excision is the greatest. It should prove to lower the incidence of complications of hemispherectomy techniques. We recommend peri-insular hemispherotomy in hemispherectomy candidate when the ventricle on the operated side is diffusely enlarged. 
E5.

\section{Surgical Treatment of Epilepsy Associated with Cortical Dysplasia}

R. LeBlanc, D. Tampieri, W. Feindel, F. Andermann, F. DUBEAU and Y. RobITAILle (Montreal, Quebec)

Background: Disorders of neuronal migration, including cortical dysplasia, are recognized with increasing frequency by MRI in epileptic patients. We report our experience with the surgical treatment of 8 patients with focal epilepsy associated with cortical dysplasia.

Patients \& Methods: Focal cortical dysplasia was the only lesion in 2 cases, was associated with more extensive, uni- or bi-lateral periventricular nodular heterotopia in 3 cases, and with a schizencephalic cleft in 3 others. The 7 males and 1 female, aged 20 - 46 years, had been epileptic for $1-28$ years. Five had temporal lobe seizures, 1 had frontal lobe seizures, 2 had frontal and temporal seizures, and 6 had secondary generalization. MRI demonstrated the pathology in all cases, and there was concordance of clinical, radiological, electrographical and neuropsychological data. Five patients underwent anterior temporal resection and amygdalohippocampectomy, 1 had a frontal resection, and 2 had a frontotemporal resection. Results: Follow-up is from $1-6$ years: All patients are on medication. One patient is seizure-free and 6 others have had significant reduction in seizure frequency. Conclusions: Resection of a well-defined epileptic focus, especially in the area of dysgenesis is included in the resection, can produce worthwhile improvement in seizure frequency without neurological or psychological deterioration.

\section{E6.}

\section{Surgery of Frontal Lobe Epilepsy Results in 109 Patients}

\section{D.LACERTE, A. Olivier and F. Andermann (Montreal, Quebec)}

The authors present a series of 109 surgically treated cases of intractable frontal epilepsy. All patients were operated by the same surgeon. 97 patients were available for follow-up, including 88 patients with at least 1 year follow-up (range $1-16$ years; mean 5.8 years).

Patients were classified according to the etiology, the side of resection and the type/extension of resection. A sub-group of patients were investigated with depth electrodes before the resective procedure.

Analysis of overall outcome (patients with a minimal 1 year follow-up) revealed a $66 \%$ success rate (class I to III, according to Engel's classification). The best indicators for a good outcome were a larger resection, the presence of an epileptogenic lesion (lesionectomy group) and a frontopolar localization of the epileptogenic focus and resection. The effect on the outcome of an additional anterior callosotomy for selected cases, and of a previous depth electrode implantation, are discussed. Also the role of high-field MRI in the pre-operative diagnosis of specific lesions for example localized forms of cortical dysplasia, is also emphasized. New strategies in the surgical management of frontal lobe epilepsy are discussed, including tri-dimensional imaging and frameless stereotactic system which are particularly useful for anatomical mapping of eloquent speech and motor areas and for surgical guidance toward small "invisible" epileptogenic lesions.

\section{F. RADIOSURGERY}

F1.

Mortality and Morbidity of Stereotactic Biopsy of IntraAxial Brain Lesions

M. Bernstein, R. Tasker, A. Lozano and A. Guha (Toronto, Ontario)

507 image-guided stereotactic biopsies performed by four neurosurgeons for intra-axial brain lesions were critically analysed regarding morbidity and mortality of the procedure. Complications were suffered by 39 patients (7.7\%).

Eleven patients (2.2\%) died following the procedure, all from intracranial hypertension: two from subarachnoid hemorrhage, six from intracerebral hematoma, and three from increased edema, without hemorrhage. Ten patients harboured malignant tumours and in one patient a definitive diagnosis was not established.

Another 28 patients (5.5\%) suffered increased neurological deficit due to intralesional hemorrhage (24), subarachnoid hemorrhage (1), subdural hematoma (1), or in the absence of hemorrhage (2). In $23(4.5 \%)$ the deficit was mild and/or transient and had no significant impact on the patient's functional status or length of survival. In the other $5(1.0 \%)$, a major neurological deficit was incurred, markedly affecting the remainder of the patient's life.

In summary, $2.2 \%$ died, $1.0 \%$ had major morbidity, and $4.5 \%$ had minor morbidity following stereotactic biopsy. Most patients selected for stereotactic biopsy harbour deeply situated malignant and/or locally aggressive intra-axial pathology and one would expect a certain level of morbidity associated with blind sampling of such lesions. Honest and rigorous reporting is imperative in accurately assessing the risk associated with surgical interventions in our patients.

F2. Radiosurgery for Acoustic Neuromas: The Toronto
Experience

M.L. Schwartz, C. Young, P. O'Brien and R. Ramani (Toronto, Ontario)

Since October, 1991, 10 patients have received stereotactic radiosurgery for acoustic neuromas. We believe that the primary treatment for acoustic neuromas is microsurgical excision. Radiosurgery is offered to patients who are elderly, infirm, or who have undergone incomplete resection(s) and whose tumors are shown to be growing on serial imaging. Average size of treated tumors was $3 \mathrm{~cm}$. Using a $6 \mathrm{MV}$ linear accelerator, a dose of 12 Gy at the $40 \%$ isodose contour was given to the edge of the tumor in all cases. Volumetric measurements were made at the time of radiosurgery and at 6 month intervals thereafter. Eight patients had follow-up imaging. All tumors show loss of 
central enhancement suggesting necrosis. Four tumors are smaller by a mean decrease in volume of $15 \%, 3$ are unchanged in size and one tumor is slightly larger. Three patients had increased gait ataxia. One developed typical trigeminal neuralgia. Only one patient had serviceable hearing prior to treatment and this has declined slightly. There were no other complications. Ten patients will be eligible for 6 month follow-up by June 1994.

\section{F3.}

\section{Should Radiosurgery Play a Role in Treating Solitary Cerebral Metastases?}

J.L. Caron, L. Souhami, J.G. Villemure, J.P. Bahary, E. Podgorsak, P. Bourgouin, B. Clark and C. Pla (Montreal, Quebec)

Purpose: Surgical removal of solitary cerebral metastatic tumors followed by whole brain radiation achieves longer survival when compared to radiation alone. What do we have to offer when surgery is not an option or when we are dealing with locally recurrent disease? We have explored the biological and radiological effects of stereotactic radiosurgery in previously treated recurrent cerebral metastases to determine the role, if any, this therapeutic option could have in managing this form of cerebral malignancy.

Methods: From June 1988 to December 1993, 56 patients harboring 58 tumors were treated. The out-patient procedure consists of a single fraction radiosurgical dose delivered to the tumor edge using a Linac based dynamic rotation technique developed at McGill University. The median age of the patients was 55 years (range 41-77). The median tumor size was $25 \mathrm{~mm}$ (range 10-60 mm). The median dose was of $20 \mathrm{~Gy}$ in a single fraction (range 10-25 Gy) delivered to the $90 \%$ isodose surface in 52 cases $(85 \%)$, to the $70 \%$ in 4 cases $(7 \%$ ) and to the $50 \%$ isodose surface in 2 cases ( $3 \%$ ).

Results: The patients were evaluated by serial CT and clinical exams at 1 month post-treatment and every 3 months subsequently until systemic disease took over or treatment failure. 46 lesions (79\%) had a complete or partial radiological response, $10(19 \%)$ did not change and $2(3 \%)$ failed inside the treated volume. The clinical response followed closely the radiological response.

Conclusion: This experience establishes the positive role of radiosurgery for small solitary cerebral metastatic tumors. It is now necessary to establish by randomized clinical trials the definite place it should have in the armamentorium of therapeutic options.

\section{F4.}

Frameless Stereotactic Neurosurgery: Clinical Experience Using The ISG Wand.

M.W. McDermott, P.H. Gutin, M.S.B. Edwards, N.M. Barbaro, W.P. Dillon, and C.B. Wilson (San Francisco, California)

In planning and carrying out an intracranial procedure, a surgeon must presently conceptualize the three-dimensional (3D) anatomy of the lesion to be approached using two-dimensional
(2D) information from CT, MRI or angiographic images. Visual and spatial orientation to the lesion within the intracranial compartment can be maintained only be reference to known anatomical landmarks and their relationship to the lesion. Intraoperative adjuncts currently in use to assist with this localization include ultrasound, electrocorticography and frame-based stereotactic systems. Frameless stereotactic systems have been developed which relate both $2 \mathrm{D}$ and $3 \mathrm{D}$ information back to the surgeon in real time. The ISG Viewing Wand is a freely movable, articulated, position sensing arm that is connected electronically to a computer and video terminal containing 2D CT or MRI images and $3 \mathrm{D}$ reconstructions. This device is under clinical evaluation at UCSF and at present (12/29/93) experience has been gathered from its use in 25 patientts. Diagnoses pre-operatively were: glioma (14); cavernous angioma (5); metastases (2) and; 1 each of chordoma, meningioma, hemangioma and radiation necrosis. Eighty percent $(20 / 25)$ of patients were scanned the day of surgery, 4 one day before and 1 three days before. MRI imaging was used in 18 patients, CT in 7 . The mean/median times for spatial registration of 2 data sets (fiducials/anatomic-skin) were 17.5/14 minutes (range 5-70 minutes). The Wand was used an average of 5 times during surgery. There were 4 technical problems with the system, 3 of which prevented its use. There were no cases of a break in the sterile field, nor adverse patient outcome as a result of use of this device. Seventy-five percent of surgeons felt the system was a valuable addition to surgery, especially for small subcortical or ganglionic lesions, with a median ease of use rating of $8 / 10$ ( $1=$ not useful; $10=$ invaluable). This experience will be updated to $4 / 94$ at presentation.

\section{F5.}

\section{The Impact of Stereotactic Radiosurgery Within University Neurosurgical Practice in the United States}

\section{KondziolKa and L.D. LunSFord (Pittsburgh, Pennsylvania)}

We have evaluated the 6.5 year impact of a Stereotactic Radiosurgery (gamma unit) program on general neurosurgical practice at our institution (University of Pittsburgh). Since the installation of this initial United States unit (and fifth worldwide), over 20 units nationally, and over 60 units worldwide became operational. In addition, hundreds of linear accelerators were also modified. As a technique that provided neurosurgical management without general anesthesia, an incision, or a prolonged hospital stay, radiosurgery proved attractive to both patients and referring physicians. The purpose of this report was to evaluate the evolving role of radiosurgery within our departmental surgical practice at a tertiary care academic center.

Radiosurgery was selected for patient care in between 5.2 and $8.2 \%$ of patients (total $=1250$ ) undergoing neurosurgery. Over $90 \%$ of vascular malformations and $50 \%$ of acoustic tumors underwent radiosurgery instead of microsurgery. Over $80 \%$ of patients with brain metastases at our center had radiosurgery rather than open resection. Radiosurgery was an effective alternative therapy for primary, recurrent or residual skull base tumors $(n=480)$. Radiosurgery was incorporated into a protocol for small volume malignant gliomas after infusion chemotherapy and fractionated radiotherapy. Average length of hospital stay was reduced from 36 hours in 1987 to 24 hours 
(1991; with same day admission), and then for most patients to 8 hours (1993; with same day discharge). Reduction in hospital costs, a rapid return to employment status, long term efficacy, and reduced morbidity, have maintained high numbers of patients treated (average, 230 patients per year for 2 neurosurgeons).

Stereotactic radiosurgery is a multi-disciplinary treatment strategy with a growing impact on the neurosurgical management of intracranial tumors and vascular malformations. The roles of this technique are being evaluated in prospective randomized trials for malignant tumors, and in economic and clinical outcome comparisons for benign tumors. The principles and practice of radiosurgery must be included in the contemporary training of neurosurgeons and radiation oncologists. Although a program may represent an initially high start-up cost, our longterm analysis indicates that radiosurgery will continue to result in a major reduction in the overall individual and society costs of high-technology health care delivery at major medical centers worldwide.

\section{F6.}

\section{"Failed" Stereotactic Biopsy in a Series of 507 Cases}

T. M. Soo, M. Bernstein, R. TAsker, A. Lozano and A. Guha (Toronto, Ontario)

Failed stereotactic biopsy is one in which a definitive diagnosis is not achieved based on the tissue obtained. Certain important differential diagnoses may have been effectively excluded, but a specific diagnosis dictating further specific therapy has not been established.

We examined 507 consecutive image-guided stereotactic biopsies for intra-axial mass lesions performed by 4 neurosurgeons. Approximately $25 \%$ of the cases were performed using the Leksell system while $75 \%$ were done using the BrownRoberts-Wells system. The majority of patients harboured malignant tumours (glioma>metastasis $>$ lymphoma). Approximately $15 \%$ of lesions were of specific inflammatory or infectious etiology.

No definitive diagnosis was achieved in 38 cases $(7.5 \%)$. In most cases tissue was obtained but was "non-specific inflammatory and/or gliotic". In a very small number of cases the lesion could not be penetrated because of its unusually firm consistency and no tissue was obtained. In a small minority of cases the lesion was frankly missed yielding normal brain.

The diagnostic success of a stereotactic biopsy probably depends largely on the histological nature of the lesion, and the optimal targetting and sampling of the lesion. Failure to achieve the diagnosis is uncommon with solid neoplasms but is more common in lesions of inflammatory etiology.

\section{G. GENERAL NEUROLOGY}

\section{G1.}

Creating a "Brain and Behaviour Unit" for Neurosciences in a Problem Based Curriculum.

R.A. Purdy, B. Holmes, D. Kaufman, M. Michalon, D. RASMUSSON and J. RUTHERFORD (Halifax, Nova Scotia)
Recently, problem based learning has become popular as a teaching method in undergraduate medical curricula. This method de-emphasizes lectures, promotes self-directed learning, is presented in the context of cases, and has resulted in the evolution of interdisciplinary courses.

In the fall of 1993, Dalhousie University embarked on year two of its Case Oriented Problem Stimulated (COPS) undergraduate curriculum. The first unit in second year, the Brain and Behaviour Unit, consisted of ten cases (1/week), seven neurological and three psychiatric. The Unit was designed to chiefly teach basic neurosciences, mainly neuroanatomy and neurophysiology, although neuropharmacology, therapeutics, and clinical aspects of neurology and psychiatry were introduced. Laboratory sessions in neuroanatomy were included.

These cases, based on real patients, were developed by Dalhousie faculty. Learning objectives, tutor guides, and resource materials were developed for each case. The cases were supplemented by a maximum of three lectures per week, and by clinical demonstrations. Evaluation was case based. Student and faculty evaluation of the Unit was very positive.

We overview the conceptual approaches and logistical problems encountered in developing this new Unit for teaching neurosciences. We emphasize that success in Unit evolution requires the full cooperation of clinicians, basic scientists and educators.

G2.

Vigabatrin in Uncontrolled Complex Partial Seizures in Adult Patients with Epilepsy: a Double-Blind, PlaceboControlled, Dose-Ranging Study

A. Guberman, J. Bruni, C. Desforges, M. Jones, M. A. Lee, R. Mclachlan, A. Ogunyemi, N. Pillay, S. Purves, M. Sadler, E. Starreveld, N. Sussman, L. Vachon and D. WEAVER (Laval, Quebec; Vancouver, British Columbia; Calgary; Edmonton, Alberta; St. John's, Newfoundland; Winnipeg, Manitoba; Halifax, Nova Scotia; Kansas City, Michigan; Ottawa; Toronto; London; Kingston, Ontario)

Vigatrin ( $\gamma$-vinyl-GABA, Sabril ${ }^{\circledR}$ ) (VGB) was studied in patients with uncontrolled complex partial seizures with or without secondary generalization, in a multicentre, double-blind, parallel group design. Following a 12 week baseline period, 111 patients (age: 18-50) were randomized to receive a placebo (PBO) $(\mathrm{N}=53)$ or VGB $(\mathrm{N}=58)$ for 8 months, as add-on to their current antiepilepsy medication regimen. The treatment was initiated at $1 \mathrm{~g} /$ day and the dose then adjusted to $2-4 \mathrm{~g} /$ day depending on therapeutic response. VGB led to a statistically significant decrease $(p=0.001)$ in the monthly mean seizure frequency (median: 7.3 to 3.5 ), as compared to PBO (median: 8.2 to 6.0 ). A reduction in seizure frequency of $\geq 50 \%$ was observed in $48 \%$ of the patients on VGB, as compared to $26 \%$ on PBO (P $=0.02$ ). The most frequent adverse events observed in the VGB group included headache, fatigue, vision and memory disturbances, dizziness and weight increase. Premature discontinuation due to adverse events occurred in four cases $(8 \%)$ on PBO and six $(10 \%)$ on VGB. No clinically significant changes were observed in the laboratory tests, MRI's, visual and somatosensory evoked potentials, cognition' and abilities, while minor changes 
were observed in mood scales. This study demonstrates that VGB is well tolerated and effective in improving seizure control when added to a current antiepilepsy medication regimen.

\section{G3.}

\section{Progression of Carotid Atherosclerosis in Diabetics}

L.T. Smurawska, C.F. Bladin, A.V. Alexandrov, J. Silverberg, P.N. Burns and J.W. Norris (Toronto, Ontario)

Diabetes mellitus (DM) produces accelerated atherosclerosis with increased risk of vascular complications including stroke. The carotid arteries are easily accessible to ultrasound evaluation and are an established marker for stroke and myocardial ischemia. We measured carotid intimal-medial thickness (IMT) and assessed early plaques using high-definition ultrasound imaging. We also documented the risk factors such as hypertension, smoking and type and duration of diabetes.

IMT is significantly thicker in diabetics matched to controls by age and sex ( $p<0.01$ ), and thicker in IDDM patients compared to NIDDM $(p<0.01)$. There were more stenosing and non-stenosing plaques in diabetics, and this was directly related to the duration of diabetes. IDDM patients had more plaques then NIDDM ( $68 \%$ vs. $57 \%$ ). ITMT is thicker in patients with plaques compared to those without plaques $(p<0.001)$.

Substantial vascular changes occur in the carotid artery walls very early and these relate to the duration and type of diabetes.

\section{G4.}

Canadian Collaborative Project on Genetic Susceptibility to Multiple Sclerosis.

A. D. Sadovnick, G.C. Ebers and N. Risch (Vancouver, British Columbia; London, Ontario; New Haven, Connecticut)

The overall objective of this proposal is to elucidate the pathogenesis of MS. The research will draw on an already established and productive database consisting of over 20,000 Canadian MS patients from all the Canadian MS Clinics. The research is a comprehensive study of genetic susceptibility to MS, incorporating both genetic epidemiological and molecular genetic approaches.

Data collection is being carried out at each clinic site, using a standardized screening questionnaire which will be administered directly to the MS patient and other relevant family informants by local interviewers.

The purposes of this presentation are: (1) to inform Canadian neurologists about the structure and objectives of this major Canadian research initiative study and (2) to present preliminary results on the population-based survey of over 20,000 MS patients with respect to the distribution of familial MS. We will present data on the overall familial occurrence of MS among various categories of relatives such as parent/child, siblings, half-siblings, first cousins as well as non-biologically related individuals who cohabitate, e.g., spouses, adopted siblings.
G5.

An atypical MS-like Syndrome Associated with Silicone Breast Implants or Silicone Fluid Injections into Breast.

\section{B. Ostermeyer Shoaib and B.M. Patten (Houston, Texas)}

Silicone breast implants (SBI) have been associated with systemic autoimmune and rheumatologic diseases in an increasing number of reports. Silicone and silica (up to $30 \%$ of the implant) have been shown to be cytotoxic and immunostimmulatory agents and can cause autoimmune reactions. We report 12 women who developed a multiple sclereosis-like syndrome at a median age of 39 years (range 27-50 years). The median latency between augmentation and onset of symptoms was 6 years (range 3 months-13 years). Eleven patients had received silicone-gel breast implants and one patient had free silicone fluid injections into her breasts. All had a history and neurological findings compatible with multiple sclerosis. Additional symptoms included myalgia $(n=11)$, joint stiffness $(n=11)$, memory deficits $(n=9)$, Sicca complex (dry eyes and dry mouth) $(n=8)$, arthralgia $(n=6)$, headache (5), joint swelling $(n=4)$, hair loss (4), Raynaud's phenomena $(n=5)$ and rash (4). All had a spinal tap done and all had oligoclonal bands. Eleven had visual evoked responses measured and all were found to be delayed ( 8 bilateral, 2 on the right, 1 on the left). All had a MRI of the brain and 11 of 12 had multiple white matter lesions and one had a normal study. Nine of 12 had autoantibodies. Our patients developed a syndrome of multiple sclerosis along with the neuromuscular and rheumatologic symptoms previously reported in patients with "Adjuvant Breast Disease".

G6.

Ischemic Oculomotor Nerve Palsy in Rheumatoid Arthritis.

\section{A.O. OGunYemi (St. John's, Newfoundland)}

Ischemic oculomotor nerve palsy is characterized by acute onset of symptoms and signs related to dysfunction of the oculomotor nerve with sparing of pupillary reflexes. It is rarely documented in patients with rheumatoid arthritis. We here, describe the course of the syndrome in a patient with rheumatoid arthritis.

This 47-year-old woman developed acute onset of double vision, nausea and right periorbital pain. Four days later, she noted marked drooping of the right eyelid. Physical examination revealed complete ptosis, weakness of superior rectus and inferior oblique of the right eye and vertical diplopia. Pupillary reflexes were normal.

She has had rheumatoid arthritis for about 10 years. The visual symptoms began almost 3 weeks after methotrexate, taken for 2 years, was discontinued. CBC, blood glucose, serum electrolytes, liver enzymes, C3, C4 and cryoglobulins; CT scan of brain and orbits and CSF examinations were all normal. Rheumatoid factor was negative. ESR was elevated at $49 \mathrm{~mm} / \mathrm{hr}$. She was treated with prednisone with complete resolution of symptoms and signs in 2 months.

We conclude that ischemic oculomotor nerve palsy may rarely complicate the course of rheumatoid arthritis. The prognosis is good for full recovery. 


\section{H. BEHAVIORAL NEUROLOGY}

H1.

\section{"Radial Neglect" is Caused by Vertical Neglect of an Internal Representation}

\section{A. KIRK and C.A.J. BoYLE (Saskatoon, Saskatchewan)}

Several patients with vertical neglect have been described. Those misbisecting vertical lines above midpoint tend to misbisect radial lines distal to midpoint and those misbisecting vertical lines below midpoint misbisect radial lines proximal to midpoint when radial lines are presented flat on a table below eye level. However, a page on a tabletop is generally thought of as having a "top" at its distal edge and a "bottom" proximally. Jeerakathil and Kirk demonstrated that normals are biased on line bisection toward what they consider to be the top of the line, independent of actual spatial position. We report a patient with neglect of left hemispace and inferior hemispace following a right parietal infarction. He misbisected horizontal lines significantly toward the right of midpoint. Vertical lines were misbisected significantly above midpoint, radial lines below eye level significantly distal to midpoint, and radial lines above eye level (so that the "top" of the page was now the proximal edge) significantly proximal to midpoint. Thus, as misbisection of radial lines reversed direction depending on line position, there was no consistent radial neglect. Instead, we propose that "radial neglect" on visually guided line bisection results from vertical neglect based on an internal reference frame.

\section{H2.}

Dual Deficit of Graphic Motor Pattern and Graphic Code in Apractic Agraphia

\section{C.A.J. BoYLE and A. KIRK (Saskatoon, Saskatchewan)}

A 78-year-old right handed man had difficulty forming letters since a left superior parietal infarct 13 years previously. He had normal hand dexterity, no limb apraxia, and no sensory deficit. Language was normal. Reading was normal. He could spell words aloud, type and assemble words from anagram letters, with no effect of word class, regularity, or imageability. Thus there was no linguistic deficit. Letter imagery was intact. Copying was better than writing to dictation but not normal. Writing was slow, and effortful with extra pen strokes and poor connections of the parts of letters, making identification of the letters difficult. Drawing was similarly affected. Using Margolin's model, this man had an intact orthographic buffer and physical letter code, but a dual deficit of the graphic motor pattern and the graphic code.
H3.

Predictive Value of Single Photon Emission Computed Tomography (SPECT) Scanning in Elderly Subjects with Borderline Cognitive Impairment

R. McKelvey, H. Chertkow, H. Bergman and J. Stern (Montreal, Quebec)

Biparietal regional cerebral blood flow (rCBF) abnormalities (decreased flow) documented on SPECT scan have been found in many patients with dementia of the Alzheimer type (DAT). We wished to evaluate whether the finding of such rCBF abnormalities in elderly patients with borderline cognitive impairment would predict subsequent development of dementia. We assessed 100 elderly subjects with memory impairment. Sixtytwo met research criteria for probable Alzheimer's disease. Thirty-eight were classified as "borderline cognitive impairment" and did not reach criteria for dementia. SPECT results were classified in a blinded fashion. Of the DAT subjects, $34 \%$ had normal SPECT scans, 26\% had SPECT abnormalities suggesting DAT, $27 \%$ had minor unilateral flow abnormalities, and $13 \%$ had other flow patterns. In the borderline subjects, there was a similar distribution of flow patterns, except that $45 \%$ showed normal scans and 10\% had SPECT abnormalities suggesting DAT. Twenty of the borderline patients have been followed for at least 12 months, and seven of these have developed dementia on subsequent assessment. There was no significant difference between flow patterns in those subjects developing dementia and those who did not. SPECT abnormalities in such borderline patients are not clearly predictive of development of dementia at one year follow-up.

\section{H4.}

Volumetric Brain-Behaviour Correlations in Alzheimer's Disease

D. Kidron, S.E. Black, P. Stanchev, B. Buck and M. BRONSKILL (Toronto, Ontario)

We examined brain-behaviour relations using volumetric magnetic resonance imaging at 1.5 Tesla, in conjunction with a battery of neuropsychological tests in 20 patients with Alzheimer's disease (AD) and 20 matched controls. Semi-automated bifeature segmentation of proton density and T2-weighted images was applied to derive separate estimates of grey and white matter and of sulcal and and ventricular cerebrospinal fluid (vCSF). On the lateral surface of 3D brain reconstructions, major the anatomical landmarks were used to demarcate the frontal, parietal, temporal and occipital lobes in each subject. Regional brain volumes were then correlated with performance on putative cognitive tasks for these regions, by multivariate methods which partialled out the effects of age, education and performance on global cognitive scales.

vCSF colume of the whole brain was significantly larger in AD subjects compared to controls $(p<0.003)$ and correlated significantly with performance on overall dementia rating scales $(\mathrm{p}<0.004)$. The difference in regional vCSF was most significant for the temporal and parietal lobes, especially on the left. Frontal vCSF volume differed significantly only on the left.

These methods provide an objective technique for quantifying topographical changes in normal aging and $\mathrm{AD}$ in correlation with cognitive performance. 
H5.

Septal Stimulation in Aged Rats Improves Performance in a Task of Working Memory

\section{J. Turnbull, F. Jiang and R. Racine (Hamilton, Ontario)}

We have previously demonstrated that electrical stimulation of the perforant path at the theta frequency improves performance in a test of working memory in rats with prior bilateral fornectomy. We now report that electrical stimulation at the theta frequency of the perforant path, and especially of the septum, imporves perfomance in aged rats in a test of working memory in a random cross over design. These results strengthen the supposition that intrinsic synchronizing rhythms are implicated in normal learning and memory, and raise the possibility that certain disease states may be at least partially attributable to disrupted synchronicity. Possible mechanisms and implications will be discussed.

H6.

\section{A Case of Dementia Secondary to Arteriopathic Leucoencephalopathy}

C. Chayer, A. Robillard, Y. Robitaille, J. Boileau, J. Lachapelle, P. Laplante, S. Rousseau, L.E. Roy and J. Teitelbaum (Montreal, Quebec)

Objective: We present the case of a sixty-year-old man with peripheral Buerger disease and a rapidly evolutive subcortical dementia.

Background: Recent publications detailed cases of leucoencephalopathy with subcortical stroke-like episodes and subcortical dementia in patients without vascular risk factors. Some of them proved to have a familial incidence with autosomal dominant inheritance.

Design Method: The patient had clinical evaluation with neuropsychologic testing, biochemical and radiological studies and a cerebral biopsy. A familial history and genetic marker research focusing on chromosome 19 was done.

Results: Neuropsychologic testing showed a cortico-subcortical dementia with massive deterioration at one year. Radiologic studies demonstrated a predominently white substance disease. Vascular abnormalities tend to be confined to small caliber arteries as previously described in familial arteriopathic leucoencephalopathy. Neither evidence of atherosclerosis, embolic or genetic disorders, nor cerebral Buerger disease were found. Genetic study results are still pending.

Conclusion: Presentation and accelerated evolution of this cortico-subcortical dementia correspond clinically and pathologically to arteriopathic leucoencephalopathy, in a patient with coexisting peripheral Buerger disease. A familial incidence in this case still awaits confirmation.

\section{CEREBROVASCULAR SURGERY}

I1.

\section{Giant Carotid-Ophthalmic Aneurysms: Results of Surgical Management in 113 Cases}

\section{G.G. Ferguson and C.G. Drake (London, Ontario)}

The total experience with carotid-ophthalmic aneurysms at University Hospital in London, Ontario is now slightly in excess of 300 cases. Of these, 113 have been of giant size. These cases confirm our earlier observations that giant carotid-ophthalmic aneurysms occur especially in women (92\%), with a unique tendency to obtain giant size without rupture $(81 \%)$ and with a marked propensity to present with evidence of optic apparatus compression $(72 \%)$

Eighty-eight patients underwent a direct approach with clipping of the aneurysm in 85 cases and exploration only in 3 cases. Twenty-five patients were treated with an indirect approach: proximal carotid artery occlusion in 20 cases and a trapping procedure in 5 cases.

A satisfactory result was obtained in $89 \%$ of these cases; an unsatisfactory result in $11 \%$. Of 7 deaths, 2 were the result of hemispheric infarction from inadvertent carotid occlusion with clipping, 1 from recurrent hemorrhage when a clip slipped, 1 as a direct result of hemorrhage in a poor grade patient, 1 from a defective pump run during cardiopulmonary bypass, and 1 from hemorrhage following exploration and before a planned carotid occlusion. There were 6 poor results, most often the result of unintentional carotid occlusion with direct clipping of the aneurysm. Overall visual function was improved in $36 \%$ and worsened in $28 \%$.

I2.

\section{Skull Base Approaches for Management of Aneurysms of the Posterior Circulation}

M.G. Hamilton, G. Thompson, J. Zabramski and R. Spetzler (Calgary, Alberta; Phoenix, Arizona)

Aneurysms of the posterior circulation account for between $10 \%$ to $15 \%$ of all intracranial aneurysms and generally have a natural history similar to that of anterior circulation aneurysms. However, the complex anatomy of the vertebrobasilar system, brain stem and cranial nerves and the bony confines of the posterior fossa can create a considerably greater amount of technical difficulty than encountered in treatment of aneurysms of the anterior circulation. Skull base approaches can provide significant improvement during surgical treatment of many posterior circulation aneurysms. The majority of basilar bifurcation aneurysms can be effectively treated with a subtemporal or pterional-transylyvian approach. However, aneurysms arising from a high basilar bifurcation can be more effectively approached with the aid of the orbitozygomatic pterional-transylvian approach. Aneurysms of the basilar trunk can be safely approached through transpetrosal, the combined supra- and infratentorial (combined), or far lateral surgical approaches. Aneurysms of the vertebral artery and PICA can be safely approached via the far lateral approach. A total of 115 posterior circulation aneurysms have been treated in the last five years, utilizing the basic principle of optimized surgical access, including when necessary the 
utilization of skull base techniques. In addition, adjuvant techniques, including cerebral protection, temporary vessel clipping and cardiac standstill have been used to aid in the treatment of these lesions. A review of a management strategy for the surgical treatment of posterior circulation aneurysms will be presented.

\section{I3.}

Tri-Dimensional, Computed Tomographic Angiography (3D-CT angiography); A Useful Adjunct for the Assessment of Complex Aneurysms

R. LeBlanc, D. Tampieri, J. Oleszek, R. Pokrupa, J.-G. Villemure and D. MELANSON (Montreal, Quebec)

Background: We described our technique and results of dynamic, reconstructed, tri-dimensional computed tomographic angiography (3D-CT angiography) in our first 12 patients with complex aneurysms producing subarchnoid hemorrhage or compression of neighbouring structures. We assess the role of this technique in treatment planning.

Methodology: A dynamic, infused CT scan producing $1.5 \mathrm{~mm}$ cuts of the area of interest was performed and the two dimensional images were reconstructed in three dimensions using the ISG Allegro (Toronto, Canada) system. Results were compared to intraarterial digital subtraction angiography. Results: All aneurysms wer demonstrated by 3D-CT angiography. The neck of the aneurysm, its relationship to the parent artery and associated branches, and its relationship to surrounding bony structures, such as the anterior clinoid, were also demonstrated. This was especially useful for large and giant aneurysms and for ophthalmic aneurysms. Conclusions and Discussion: 3D-CT angiography is useful in the evaluation of aneurysms especially when the local anatomy is obscured or distorted by its mass or by the anterior clinoid. Aneurysms as small as $3 \mathrm{~mm}$ and partially treated, acrylic coated aneurysms which could not be completely assessed otherwise have been demonstrated by this technique.

\section{I4.}

\section{Endovascular Treatment of Basilar Tip Aneurysms Using Electrolytically Detachable Coils}

C.G. McDougall, V.V. Halbach, R.T. Higashida, C.F. Dowd, G.P. Teitelbaum and D.W. Larsen (San Francisco, California)

Between August, 1991 and December 1993, 19 patients with basilar tip aneurysms were treated using electrolytically detachable coils. The most frequent presentation was subarachnoid hemorrhage. All patients were referred following neurosurgical assessment and exclusion as candidates for surgical clipping. Reasons for exclusion from surgical clipping included failed surgical therapy, major systemic illness, clinical judgment and anticipated surgical/technical difficulty.

In the majority of patients greater than $90 \%$ occlusion was achieved using this technique however a small residual aneursym neck was a frequent occurrence. This situation arose because complete elimination of the neck would frequently risk compromising the parent artery lumen. Alternatively in some cases only partial occlusion was possible because of origin of the posterior cerebral or superior cerebellar arteries was from the wall of the aneurysm.
One patient who presented following subarachnoid hemorrhage as Hunt and Hess grade $\mathrm{V}$ failed to show any recovery following treatment and eventually died. One patient suffered major permanent morbidity from thrombosis of the basilar tip region a few hours following coil placement.

It is felt that this technique is a reasonable alternative to conventional surgical treatment for basilar tip aneurysms when conventional measures are judged unsuitable. Greater experience and long term follow up is mandatory.

\section{I5.}

\section{Pulsation - Pressure Relationship in Experimental Saccular Aneurysms}

\section{P. NovaK, R. Glikstein and G. Mohr (Montreal, Quebec)}

Purpose: To evaluate the relationship between the aneurysmal pulsation and intra- aneurysmal pressure in an experimental model of intracranial aneurysms.

Material and Methods: Bifurcation (BAn) and lateral aneurysms (LAn) were produced in 15 mongrel dogs (weight 20 - $35 \mathrm{~kg}$ ). The aneurysmal dome was constructed from a $3 \mathrm{~cm}$ segment of the external jugular vein which was sutured to the window performed on the apex of the ECA and lingual artery (for the BAn) or on the EC (for the LAn). The following signals were recorded and analysed: intra-aneurysmal pressure, aneurysmal pulsation (by the displacement sensor), femoral artery pressure and ECG.

Results: Aneurysms pulsate in synchrony with the intraaneurysmal pressure (for example a change of $70 \mathrm{~mm} \mathrm{Hg}$ in the intra-aneurysmal pressure displaces the aneurysmal wall by $0.125 \mathrm{~mm}$ for a BAn of $1.5 \mathrm{~cm}$ in size). LAn pulsate maximally at the inflow zone with decreasing of the pulsation range towards the outflow zone. There is a threshold pressure beyond which the aneurysm can not expand. The pressure-pulsation curve showed that the aneurysm exhibits the property of hysteresis (for the same pressure the aneurysm is smaller during the ascending portion of the blood pressure wave).

Conclusion: The presence of hysteresis indicates that the aneurysm is subject to the accelerated degeneration since it is known that hysteresis results in the degeneration of connective tissue, predominantly of the elastic fibres. We hypothesize that the aneurysm is prone to rupture if the intra-aneurysmal pressure exceeds the threshold level.

\section{I6.}

Pharmacological and Morphological Effects of Transluminal Balloon Angioplasty on Vasospastic and Normal Canine Basilar Artery

\section{P. Chan, J.M. Findlay, B. Vollrath and D. Cook (Edmonton,} Alberta)

Transluminal balloon angioplasty (TBA) is a promising alternative treatment for cerebral vasospasm following aneurysmal subarachnoid hemorrhage (SAH). It is effective in cases resistant to standard therapy. The mechanism of action of TBA in cerebral vasospasm is poorly understood. We examined pharmacological and morphological changes after in vitro TBA (using a silicone 
microballoon catheter) in canine basilar arteries. 10 normal dogs and 10 dogs with SAH (double SAH model) were studied. Vasospasm in the SAH group was confirmed by angiography, and the animals were killed on the seventh day following the first SAH. Prostaglandin $\mathrm{F}_{2} a$, noradrenaline, and serotonin were used as vasoconstrictors, and bradykinin and calcium channel ionophore were used as vasorelaxants. Morphological analysis was performed with scanning electron microscopy before and after chemical digestion of either the connective tissue or cellular components of the vessel wall. We found that in normal and vasospastic arteries treated with TBA: 1) the response to the vasoconstrictors and the endothelium dependent vasorelaxants was either significantly diminished or abolished $(\mathrm{p}<0.001) ; 2)$ the endothelium and smooth muscle cells appeared intact although corrugations in the intima of the vasospastic arteries were flattened. We conclude that TBA impairs vasoconstriction and endothelium dependent relaxation of normal and vasospastic canine basilar arteries in the absence of any significant structural alteration.

17.

\section{A Comparative Study of the Surgical Management Between} Ruptured and Unruptured Intracranial Aneurysms

G.A. Dix, W. Gordon, I.S. Sutherland and G.R. SuTherland (Calgary, Alberta; Winnipeg, Manitoba)

The management of unruptured, intracranial aneurysms remains a contentious issue, despite recent literature advocating elective surgical intervention.

In this retrospective review, the outcome of 119 patients with 161 ruptured and unruptured intracranial aneurysms was evaluated. Of the 89 ruptured aneurysms that were surgically ablated, 65 had an excellent outcome, 11 were good, 3 moderate, 1 poor and 8 patients died post-operatively. In 6 cases incidental, ruptured aneurysms were clipped concurrently without affecting the outcome. Patients who presented with unruptured aneurysms fell into two categories: symptomatic and asymptomatic. Outcome assessment in these cases was correlated to pre-operative neurological status. Of the 45 asymptomatic aneurysms 43 had an excellent post-operative result whilst I was good (persisting right incomplete 3 rd nerve palsy) and 1 was moderate (left hemiparesis). In the symptomatic group, 12 aneurysms were diagnosed as a result of mass effect and post-operatively all 12 cases were accorded excellent outcomes.

In view of the outstanding results that follow surgical intervention of unruptured aneurysms compared to the morbidity/ mortality associated with subarachnoid hemorrhage following aneurysmal rupture, the prophylactic surgical treatment of unruptured aneurysms is advocated in all clinically appropriate patients.

I8.

\section{Angiographic and Biological Screening of Familial Cerebral Aneurysms}

R. LeBlanc, D. Melanson, D. Tampieri and R.D. GuttmanN (Montreal, Quebec)

Background: The mode of transmission and prevalence of familial cerebral aneurysms is unknown thus the management of asymptomatic family members is empiric. We report our prospective studies to establish the prevalence of aneurysms in families and our attempts at finding a biological marker for these based on the HLA system. Methods: We performed elective intra-arterial digital subtraction angiography or MRA in 18 asymptomatic siblings from 6 families, and corelated the presence and absence of aneurysms to the HLA profile in 4 of these families to identify a putative specific antigen or genotype associated with familial cerebral aneurysm. Results: One new aneurysm and 2 infundibula were identified. Combined with the 7 previously known aneurysms the prevalence of aneurysms in sibships was established at $32 \%$ ( $40 \%$ if infundibula are considered). There was no HL antigen or phenotype associated with the presence of aneurysms. (Specifically, HLA B-7, and DR-2 did not segregate with aneurysms as some have suggested). Conclusions: The prevalence of cerebral aneurysms in sibships is $32-40 \%$. HLA typing is not useful in identifying carriers of aneurysms suggesting that a lesion of the short arm of chromosome 6 is not involved in their etiology. Until a specific biological marker is identified angiographic screening remains the only way to detect asymptomatic aneurysms in families.

\section{9.}

Combined Neurosurgery, Interventional Neuroradiology and Stereotactic Radiosurgery to Treat Large Inoperable Cerebral Arteriovenous Malformations

J.-L. Caron, J. Raymond, D. Roy, L. Souhami, M. Bojanowski, E. Podgorsak, B. Clark and C. Pla (Montreal, Quebec)

Purpose: Endovascular embolization of arteriovenous malformations is mainly used to reduce the size of larger lesions in preparation for surgical excision. The experience with stereotactic radiosurgery, on the other hand, has demonstrated its reliable ability to obliterate lesions measuring less than $3 \mathrm{~cm}$. with minimal morbidity. When screening patients for treatment we are often faced with large surgically inaccessible lesions which are not candidates for embolization or radiosurgery alone. We have therefore created a multi-disciplinary team devoted to the treatment of these lesions. Between May 1992 and October 1993 we have applied a combination of surgery and/or supra selective embolization of selected parts of the nidus to purposefully reduce the size of the malformation followed by single fraction radiosurgery of the remaining lesion to 10 patients. The techniques are offered to symptomatic patients for whom the natural history of the disorder leaves little doubt as to the eventual outcome.

Method and results: All lesions were initially greater than 3 $\mathrm{cm}$. Preliminary embolization was used alone in 5 patients, surgery alone in 3 patients and combination of both techniques in 2 to reduce the nidus to less than $3 \mathrm{~cm}$ and preferably less than $2 \mathrm{~cm}$. Using a Linac based stereotactic radiosurgical technique developed at McGill University, $20 \mathrm{~Gy}$ are delivered in single fraction to the $90 \%$ isodose line covering the remaining nidus. The patients are followed by MRI at 6 months and cerebral angiography 12 to 18 months after treatment. Preliminary results of the technique will be demonstrated. Although these patients represent a highly selected group, it seems possible to offer alternative therapeutic options in otherwise desperate situations. 
I10.

\section{Radiosurgery for Arteriovenous Malformations: The Toronto Experience}

\author{
C. Young, M.L. Schwartz, P. O'Brien and R. Ramani \\ (Toronto, Ontario)
}

Since July, 1989, 70 patients have received stereotactic radiosurgery for arteriovenous malformations of the brain. All cases were reviewed by our multidisciplinary group and radiosurgery was given as sole treatment or in conjunction with surgical or endovascular therapy. Using a $6 \mathrm{MV}$ linear accelerator, a dose of either 15 or $20 \mathrm{~Gy}$ was given to the edge of the lesion so that no eloquent neural structure received a dose of more than $15 \mathrm{~Gy}$. Of the initial 24 patients followed for at least 2 years, 12 have complete obliteration documented by angiography. Eight have had obliteration as indicated by MRI scanning only or greater than 90\% reduction as shown by angiography. Three have had the nidus diminish by less than $90 \%$ and one has had no change. Within this cohort, one patient had a seizure at 6 hours after treatment and one has developed a mild incoordination of his legs. One patient suffered a fatal hemorrhage 16 months after treatment despite a significant reduction in the size of the nidus. Two have recently undergone retreatment for small residua. By June, 1994, 38 patients will be available for 2 year follow-up.

111.

\section{Recent Results with Middle Cerebral Artery Aneurysms}

\section{J.M. FInDLAY (Edmonton, Alberta)}

A series of 47 patients with 53 middle cerebral artery (MCA) aneurysms treated over a 4 year period are reviewed. Four patients had bilateral MCA aneurysms and two patients had 2 ipsilateral MCA aneurysms. There were 30 women $(64 \%)$ and 17 men (36\%) between the ages 25-80 (mean age 58). Presenting clinical grades (Botterell-Lougheed scale) were as follows: 0 (unruptured) -17 patients (33\%), I-2 patients (4\%), II-9 Patients (18\%), III-8 patients (16\%), IV- 11 patients ( $21 \%)$, and V-4 patients (8\%). Other presenting features with rupture were hemiparesis in $13(38 \%)$, intracerebral hemorrhage in 17 $(50 \%)$, and intraventricular hemorrhage in $5(15 \%)$. Twentynine $(85 \%)$ of 34 patients with subarachnoid hemorrhage (SAH) presented with 24 hours of SAH and $23(79 \%)$ of those operated underwent clipping within 48 hours of SAH (later surgery being due to a delay in diagnosis). Rebleeding was documented in 6 patients (18\%).

Ten aneurysms (19\%) were located in the M1 segment, 4 assocated with a lentriculostriate branch and 6 associated with a temporal branch. Thirty-seven $(70 \%)$ were located at a $\mathrm{Ml}$ bifurcation, while $4(8 \%)$ were at a trifurcation and $2(3 \%)$ were located more distally at a M2-M3 bifurcation. Forty-two (86\%) of the operated aneurysms were estimated to be $10 \mathrm{~mm}$ or less in diameter and the remainder were $15 \mathrm{~mm}$ or less.

There were no deaths and no surgical morbidity in the 17 operations for unruptured aneurysms (1 DVT). Among the 34 patients with SAH, there were 10 deaths (29\%), 5 occurring early due to the primary hemorrhage and 2 due to rebleeding. Another death was due to multi-organ failure, another was due to vasospasm, and a final delayed death was a patient left vegetative from the initial hemorrhage. Using the Glasgow Outcome Scale, disability at last follow-up was severe in one patient and moderate in 11; morbidity was judged due to primary hemorrhage in 4 patients $(12 \%)$, rebleeding in 2 patients $(6 \%)$, vasospasm in 2 patients (6\%), surgical branch occlusion in 2 patients (6\%), and excision of an associated AVM in another. Good outcome (no disabilities), which was observed in a total of $13 \mathrm{SAH}$ patients $(38 \%)$, correlated strongly with presenting grade. Thirty-four $(72 \%)$ patients underwent post-op angiography and 3 nonthreatening aneurysm remnants were recognized.

\section{J. GENERAL NEUROSURGERY}

\section{J1.}

\section{K.G. McKenzie Memorial Prize for Clinical Research}

Neurologic Deterioration in the Acute Phase Post Traumatic Brain Injury

\section{S.J. Konasiewicz, R.J. Moulton and A.J. Baker (Toronto, Ontario)}

To determine if neurologic deterioration after severe head injury may be an evolutionary process that is independent of intracranial physiology in the acute phase, a multiple elimination regression analysis was performed looking at the effect of $\mathrm{CBF}$, AVDO2, CMRO2, CPP, ICP, time post injury (TPI), and initial somatosensory evoked potentials (SSEPi) on SSEPs at intervals of time during the early post-injury period (SSEPt).

Information from 24 patients with GCS $\leq 8$ (age $45.71 \pm$ 3.11 years; duration of SSEP monitoring $3.75 \pm .29$ days; means \pm SEM) was analyzed. SSEPs were calculated as the peak-peak amplitude in microvolts for a 250 millisecond trace for both hemispheres combined. When all variables were entered into the regression procedure, the model chosen to predict SSEPt depended only on TPI and SSEPi $\left(R^{2}=.54, F=96.68, p=\right.$ $.0001)$. All other variables were not statistically significant $(\mathrm{p}>$ $.05)$.

The regression model suggests that the change in SSEP activity which tends to occur post head injury may not depend on cerebral physiology early after injury. If this is the case, then the deterioration in SSEP peak-peak amplitude may represent the electrophysiological correlate of evolving axonal injury demonstrated in experimental models of head injury.

\section{J2.}

\section{Outcome from Head Injury in a Canadian Trauma Centre}

R.J. Moulton, I. Sullivan, W. Tucker, M. Cusimano and P. MULLER (Toronto, Ontario)

This paper reports the results of a prospective study of 1512 consecutive head injury patients admitted to hospital between Jan. 1, 1986 and Dec. 31, 1992. Follow-up was maintained through a multi-disciplinary head injury clinic and outcome was graded using the Glasgow Outcome Score.

The overall mortality rate (100\% follow-up) was $23 \%$ (351/1512). Among the survivors with follow-up information $(\mathrm{n}=444), 49 \%$ had good outcome, $39 \%$ were moderately disabled, and $12 \%$ were severely disabled. There were no 
vegetative survivors. The mean follow-up times for these groups were 9,14 , and 14 months respectively. In patients who did not survive the mean time to death was 10 days. Falls and gunshot injuries had the highest proportion of deaths and the lowest proportion of good/moderate outcomes $(p=0.000)$. Increasing age, low admission GCS, and pupillary abnormalities were also significantly related to outcome $(\mathrm{p}<0.0001)$. Among survivors, $71 \%$ of patients with good outcomes, $60 \%$ patients with moderate disability, and $31 \%$ of patients with severe disability were discharged home from our unit.

In the severe subgroup, the mortality rate was $49 \%$ for patients who survived emergency room resuscitation. Among survivors the rates of good outcome, moderate, and severe disability were $31 \%, 47 \%$, and $22 \%$ respectively. This compares favourably with the Traumatic Coma Data Bank data in which comparable rates for survivors were $42 \%, 26 \%$, and $24 \%$ with $8 \%$ vegetative survival. The mortality rate was $36 \%$ in that series.

Our outcome results are similar to those of a multi-centre study of head injury outcome in the U.S. The increased mortality rate in our series coincides with a reduction in vegetative and severely disabled survivors, likely as the result of more discriminating use of heroic treatment measures. Rates of good/moderate outcomes do not differ substantially between the 2 series.

\section{J3.}

\section{Subdural Hematomas Associated with Intracranial Arachnoid Cysts: a Clinico-Pathological Study}

\section{S.N. SiddiQI, J.A. DunCan, L.E. BeckeR and R.P. Humphreys (Toronto, Ontario)}

The authors reviewed 48 consecutive children with intracranial arachnoid cysts seen at the Hospital for Sick Children, Toronto, between 1980-1993. Nine of these patients were associated with intracranial subdural hematomas (SDH). Initial $\mathrm{CT}$, at presentation, showed: an intracranial arachnoid cyst and SDH in 4 patients; SDH alone in 2 patients, with follow-up CT after SDH evacuation revealing an arachnoid cyst in each case; arachnoid cyst alone with SDH occurring after cyst treatment (lumbo-peritoneal shunt, 1 patient; cyst to peritoneal shunt, 2 patients). All, except one, of the subdural hematomas occurred over the convexity; one SDH occurred at the temporal pole of the middle cranial fossa. Pre-operative angiogram, in a patient with a left middle cranial fossa arachnoid cyst, demonstrated abnormal arterio-venous shunting in the region of the subdural hematoma. Surgical treatment varied according to presentation. Eight patients remain asymptomatic to date; one patient was complicated by recurrent SDH, subsequent subdural empyema, diffuse intracranial hemorrhages, and expired. Autopsy revealed a diffuse intracranial vascular dysplasia of both the arterial and venous systems involving vessels of all sizes. This study indicates that intracranial arachnoid cysts are frequently associated with subdural hematomas, and that rarely these cases may be associated with regional abnormal arterio-venous shunting or diffuse intracranial vascular dysplasia.
J4.

Injury to the Cervical Spine in Football Players - the Neurosurgeon's Role

N.C. HILl (Winnipeg, Manitoba)

Neurosurgical consultation in football players is frequently requested following injuries to the cervical spine region in these athletes. The author's experience as a neurosurgical consultant to a professional football team over 20 years is reviewed and current recommendations regarding diagnosis and prognosis are discussed.

Injury to the cervical zygapophyseal joints, nerve roots, spinal cord and brachial plexus occur frequently in football players. Each produces a typical syndrome, described in this presentation. Overemphasis on minor injuries may result in unnecessary restrictions while failure to recognize serious condition may result in potentially serious permanent neurological disability.

Injury to the spinal cord is of particular importance. The mechanism of axial loading in the production of catastrophic spinal cord injury is re-emphasized and those conditions contributing to spinal cord neuropraxia (unrecognized instability, congenital fusion, midline disc and osteophytes, and congenital spinal stenosis) are discussed relative to injuries likely to be incurred.

Advocacy of preventative measures such as proper conditioning, rule changes and equipment modification is a further important role for the neurosurgeon in this area.

\section{J5.}

Preliminary Assessment of a Short-Stay Spinal Unit Suggests Safety and Efficiency for Most Patients Undergoing Spinal Surgery for Benign Disease

\author{
R.J. Fox, K.C. Petruk, T. Lubkey and G. MacDonald \\ (Edmonton, Alberta)
}

To improve patient flow and assess the cost effectiveness of surgery for benign spinal lesions, a short stay spinal unit was established within the neurosurgical service at the Royal Alexandra Hospital. All patients were medically assessed and received thorough pre-operative education by the resident and nursing personnel at a pre-admission clinic prior to surgery. Following discharge, patients were reassessed at 6-8 weeks in clinic and 3 month outcomes were obtained through telephone contact.

Ninety patients underwent cervical or lumbar spinal procedures through this unit since its inception in November 1992, compared to a similar group of 56 patients (control group) in the year prior. Patient age, postoperative length of stay (LOS), complication rate, and pre- and postoperative employment status were assessed in relation to these procedures. A difference in pathology, operative procedure distribution, complication rate, and three month outcome was not seen. The average length of stay was 3.8 days for the short stay group, versus 6.5 days for the control group. A difference in length of stay for laminectomy discectomy was not seen, but was significant for cervical prodecures (2-4d), lumbar laminectomy (1.5d), and microdiscectomy (2.0d), lumbar laminectomy (1.5d), and microdiscectomy (2.0d). 
Decreased length of stay for these patients equated to an annual health care savings of 50000-75000 dollars as calculated by the Hospital's finance department. The early information from this preliminary study suggests that such an approach to benign spinal surgery is associated with overall patient satisfaction, is safe, and reduces the cost burden to the health care system.

\section{J6.}

\section{Evaluation of Spinal Column and Spinal Cord Injuries by MRI}

\section{Angelov, M. Fehlings and D. Mikulis (Toronto, Ontario)}

Magnetic resonance imaging (MRI) is being increasingly used to evaluated injuries of the spine and spinal cord. To describe the pathological anatomy in these patients and to classify the patterns of signal change and their prognostic significance, we examined the MRIs in 15 patients with spinal injuries (11 M, $4 \mathrm{~F}$; age range 16-70; median initial ASIA score B; 10 cervical, 3 thoracic, 2 lumbar injuries). All patients were evaluated with a 2.0 T MR using T1, T2, and proton density imaging techniques. In selected patients, a fat-supression protocol was used to examine for ligamentous injury. Of the 10 patients with spinal cord injuries, three patterns of signal change were observed. Type A $(n=3)$ : homogeneous $\uparrow$ T1 signal; Type $B(n=5)$ : focal "bright" area on T1, surrounded by a diffuse zone of $\uparrow \mathrm{T} 2$ signal: Type $C(n=2)$ : areas of $\uparrow T 1$ and $\downarrow T 2$ signal consistent with hemorrhage surrounded by a zone of $\uparrow \mathrm{T} 2$ signal. Patients with Type A MRI changes had an excellent prognosis for neurological recovery and tended to have better initial ASIA scores. This pattern of injury may reflect acute edema. Both the Type B and $C$ patterns of injury occurred in patients with more severe injuries and were associated with less favourable prognoses. We conclude that MRI should be used to evaluate all patients with acute spinal cord injury and in selected patients with isolated spinal column trauma where accurate assessment of soft tissues such as discs and ligaments is required.

\section{J7.}

\section{The Treatment of Positional Plagiocephaly Utilizing Cranial Remodeling Orthosis (D.O.C.)}

M.G. Hamilton, C. Ripley, J. Pomatto, S. Beals, K. Manwaring, E. Joganic and D. Moss (Calgary, Alberta; Phoenix, Arizona)

Dynamic Orthotic Cranioplasty (D.O.C.) was developed to treat nonsynostotic positional head deformation. These positional deformations occur as a result of external forces exerted upon an infants craniofacial skeleton and can result in complex multistructural asymmetries affecting the cranial vault, face and skull base. D.O.C. is fabricated from a plaster of Paris impression obtained from the patient's head. From this impression, a positive model is made and the deformity is corrected to an ideal configuration with the onlay of molding material. This corrected mold serves as a template for custom band fabrication. The band applies dynamic pressure to the elevated areas while leaving space for cranial growth in depressed regions.
Between 1988 and 1993, we have employed external cranioplasty in the treatment of 161 infants exhibiting a wide range of plagiocephalic configurations. Objective linear measurements were performed on 72 patients to quantify the posttreatment correction of cranial vault and skull base asymmetry. Our anthropometric data and clinical observations show that all patients treated with D.O.C. exhibited near or complete correction of the plagiocephalic characteristics initially observed with no evidence of relapse following treatment. Dynamic Orthotic Cranioplasty is an economic and noninvasive procedure that is well tolerated by the infant. Surgery for positional plagiocephaly is seldom needed.

J8.

\section{Prothèse Gonflable (Skin Expander) Et Myéloméningocèle}

\section{J. Francoeur (Québec, Québec)}

La fermeture précoce d'une myéloméningocèle à large base s'accompagne souvent et concomittamment de nécrose, de cicatrice vicieuse, de fistule de liquide céphalo-rachidien pouvant se compliquer de méningite et de ventriculite qui chez l'enfant porteur d'une dérivation nécessite la mise en oeuvre d'un traitement très agressif dont le résultat est aléatoire.

La mobidité et la mortalité assoçiées à ces complications oblige parfois à remettre la chirurgie à un âge plus avançé. Afin de prévenir ces complications chez nouveau-né et de proçéder à la plastie tardive chez d'autres, nous avons utilisé une prothèse gonflable chez sept (7) d'entre eux.

La technique consiste à insérer par une contre-incision sous le tissu cellulaire sous-cutané, une ou deux prothèse gonflable dont le contenant peut accepter $100 \mathrm{ml}$ de sérum physiologique. L'expansion de la prothèse est obtenue par l'injection percutanée de sérum physiologique de façon stérile à raison de 15 à $20 \mathrm{ml}$ aux 4 ou 5 jours selon la qualité de la peau pour atteindre $100 \mathrm{ml}$.

La cure de la myéloméningocèle est alors faite de façon habituelle et la peau distendue est devenue facilement mobilisable pour combler la brèche cutanée quelqu'en soit la dimension. Les avantages de cette technique seront précisés à la lumière des cas traités.

J9.

Shunt-O-Gram: A Useful Adjunct to the Management of Hydrocephalic Patients

O. Vernet, J.P. Farmer, R. Lambert and J.L. Montes (Montreal, Quebec)

We studied 56 shunt-o-grams performed in 52 children presenting with symptoms of shunt malfunction where ultrasound and CT scan were equivocal. The proximal reservoir was injected with $0.5 \mathrm{cc}$ of Te-DTCA while occluding the valve manually. In 20 cases, the entire system was imaged and intraventricular reflux was present. 2 of these patients were operated on for persistent symptoms, 1 showing proximal and the other distal shunt occlusion. 16 shunt-o-grams showed abnormal distal drainage with adequate proximal reflux. 12/16 were found to show distal obstruction, shunt fracture, or peritoneal adhesions at operation. The remaining 20 shunt-o-grams did not exhibit proximal 
reflux, but showed adequate distal flow. 12/13 operated patients showed ventricular catheter obstruction, the other exhibiting overdrainage. In all groups, symptoms of non-operated patients abated spontaneously.

In conclusion, shunt-o-gram is a useful procedure in the management of patients presenting shunt-related symptoms with equivocal ultrasound or CT scan. To be considered normal, shunt-o-gram must visualize the entire shunt system, that is, there must be ventricular reflux, and the isotope must diffuse uniformly in the abdominal cavity. The presence of a proximal reservoir is mandatory for adequate interpretation of a shunt-ogram.

J10.

\section{Ventricular Isolation After Ventriculoperitoneal Shunting in Children}

\section{P. STEInBoK (Vancouver, British Columbia)}

In a retrospective review, 13 patients with a symptomatic isolated lateral ventricle and 8 with symptomatic or asymptomatic isolation of the fourth ventricle were identified among 345 children having their first ventriculoperitoneal shunt between July 1982 and June 1993.

A symptomatic isolated lateral ventricle typically occurred within five months of the original shunt, in patients without previous CSF infection, and was associated with a collapsed lateral ventricle ipsilateral to the ventricular catheter. Isolated fourth ventricles were diagnosed from 7 to 134 months after the original shunt procedure, often after multiple shunt operations, and more often associated with CSF infection. The etiology of hydrocephalus was similar in the two groups. There was no correlation between the type or pressure of the shunt valve and the occurrence of isolated lateral or fourth ventricles.

Children with a symptomatic isolated lateral ventricle presented mainly with increased intracranial pressure and were treated by shunting of the isolated ventricle. 3 of the 8 patients with isolation of the fourth ventricle were symptomatic with evidence of brain stem dysfunction. These symptomatic patients, and 1 asymptomatic patient, were treated with a fourth ventricle shunt, and the other asymptomatic children have been followed uneventfully from 19 to 88 months.

J11.

\section{Intraventricular Hemorrhage Complicating Ventricular} Catheter Revision: Incidence and Consequences

R.D. BrownleE, S.T. Myles and O.N.R. Dold (Calgary, Alberta)

A retrospective study was conducted on 105 patients who underwent shunt revision between 1986 and 1993 at the Alberta Children's Hospital (Calgary) to determine the frequency of intraventricular hemorrhage (IVH) with ventricular catheter revision. Ventricular catheter revision was performed 143 times in 74 patients. Intraventricular hemorrhage was noted intra-operatively or on post-operative computed tomographic (CT) scans on 45 occasions for an overall incidence of $31 \%$. Intraventricular hemorrhage was associated with a significantly shorter time to subsequent shunt revision $(227.33 \pm 392.24$ days) compared to catheter survival when no hemorrhage was noted (735.02 \pm 1001.11 days). Early post-operative CT scanning was performed in $71 \%$ of patients who had bloody CSF noted intra-operatively. IVH was evident on $63 \%$ of these scans. The presence of IVH on the post-operative CT scan was associated with a significantly shorter time to next revision than in those patients when IVH was not evident on the scan. The position of the catheter within the ventricle did not influence the time to first revision or the incidence of IVH. These findings raise several important questions about the prevention and management of intraventricular hemorrhage complicating shunt revision in hydrocephalic patients.

\section{K. NEUROLOGY - MOVEMENT DISORDER, HEADACHE}

\section{K1.}

Herbert Jasper Memorial Prize

Neurophysiologically Guided Pallidotomy for Parkinsonian Patients

A.M. Lozano, W. Hutchison, J.O. Dostrovsky, K.D. Davis, J. Miyasaki, A.E. Lang, J.DufF and J. Saint-Cyr (Toronto, Ontario)

The recent finding that neurons of the globus pallidus are overactive in MPTP treated parkinsonian monkeys and that decreasing the activity of this structure improves bradykinesia has been the impetus to reexplore the role of pallidotomy in humans. We now report direct physiological measures of neuronal activity in the globus pallidus of 14 awake parkinsonian patients undergoing magnetic resonance image (MRI) and stereotactic microelectrode recording guided pallidotomy. We found two populations of neurons with discharge rates of 10-20 and 30-60 per second in the lateral segment of the globus pallidus (Gpe), with neurons in the medial pallidal segment (Gpi) having a higher discharge rate of approximately 80 per second. The activity of some neurons in Gpe and Gpi was driven by movement in a direction specific manner and a crude somatotopic pattern with the lower limb represented dorsally and the arm and hand more ventrally was apparent in Gpi. The borders of Gpi, the optic tract and internal capsule were identified by recordings and by stimulation effects. the placement of the lesions was confirmed to be within GPi by MRI in all patients. The present work shows that stereotactic, imaging and neurophysiological techniques improve neural localization, increase the safety of neurosurgical procedures and open a window into the human globus pallidus to gain important information on neuronal function.

\section{K2.}

Long Term Results of Selective Peripheral Denervation (SPD) for Spasmodic Torticollis (ST)

\section{R. Benabou, C. Bertrand, P. Molina-Negro and G.Bouvier (Montreal, Quebec)}

ST is a late onset focal dystonia manifested within the third to fifth decade of life, characterized by involuntary abnormal movements and postures of the head. 
As a result of experience from former techniques, anatomic and electrophysiologic studies, SPD has been the only surgical procedure for ST in our department since 1978.

Its best indication is for restricted cervical dystonia stabilized for at least 2 years and resistant to conservative medical treatments and Botox injections. SPD aims to abolish abnormal movements in all muscles involved, while preserving innervation in those who do not participate. It consists basically in the denervation of the Sternocleidomastoid muscle, combined with contra-lateral posterior ramisetomy. Laterality and which muscles should be denervated varies according to a standard protocol.

Over 300 patients with ST went through SPD in these 15 years. Total or marked relief of symptoms with preservation of normal or nearly normal movements was obtained in $88 \%$, residual abnormal movements persisted in $10 \%$ and $2 \%$ had no improvement.

SPD is a procedure which had caused no mortality, minimal morbidity and satisfactory results that persist over the years.

\section{K3.}

\section{Ophthalmoplegia in Parkinsonism}

B.J. Stewart, A.H. Rajput and J. Ravindran (Saskatoon, Saskatchewan)

Supranuclear opthalmoplegia, with accompanying parkinsonian features, is characteristic of progressive supranuclear palsy. However, other variants of parkinsonism may also be associated with supranuclear opthalmoplegia. We report on three patients who originally presented with parkinsonism and supranuclear gaze paresis. The diagnosis of progressive supranuclear palsy was considered in all three. Each was given a trial of antiparkinsonian medications. Two of the patients demonstrated clinically significant improvements. On MRI Patient 1 demonstrated nonspecific periventricular white matter changes while patient 2 demonstrated generalized brainstem, cerebellar and cortical atrophy as well as dysgenesis of the corpus callosum. Investigation of Patient 3 revealed a brainstem lesion suggestive of a glioma. We conclude that all patients presenting with parkinsonism and supranuclear opthalmoplegia should be given a trial of anti-parkinsonian medications and be thoroughly investigated. Details of these cases, and the possible significance of our observations, will be presented.

K4.

On Drawing Impairment in Parkinson's Disease With and Without Dementia

\section{A. KIRK and A.H. RAJPUT (Saskatoon, Saskatchewan)}

Although the reported incidence varies, dementia is common in idiopathic Parkinson's disease (IPD). Various visuospatial and constructional impairments are reported in IPD with and without dementia. However, motor deficits make drawing, the simplest bedside test of constructional impairment, difficult to apply in IPD. We attempted to determine how drawing is affected by IPD and whether different deficits are seen in demented and nondemented patients. Drawings by 51 patients with IPD were compared to those of 30 healthy non-demented control subjects matched for age. All subjects performed the Western Aphasia
Battery Drawing Subtest in which they are asked to draw seven items. A standardized battery of neuropsychological tests was also administered to examine relationships between drawing impairment and other deficits. Subjects scoring below 124 on the Mattis Dementia Rating Scale (DRS) were considered to be demented. Thrity patients with IPD were thus classified as nondemented (mean DRS score 134.93) and 21 as demented (105.10). Drawings were rated independently by two raters using a scoring system previously applied in various focal and diffuse cerebral lesions. The differences between demented and non-demented IPD patients' drawings will be demonstrated and compared and contrasted with those seen in Alzheimer's disease and after focal strokes.

\section{K5.}

Fetal Dopaminergic Neural Transplantation for Treatment of Parkinson's Disease: Outcome After one Year

A. Fine, P. Gaudet, R. Holness, D. King, J. Fisk, A. Lang, B. Snow, L. Heffernan, R. Poirier, R. Redman, T. Hosokawa, C. LEOPOld, M. MacKay-Lyons, H. Robertson, F. Fingerhut and D.CALne (Halifax, Nova Scotia; Toronto, Ontario; Vancouver, British Columbia)

Fetal dopaminergic neuronal transplantation as experimental therapy for Parkinson's disease was carried out on 5 severely impaired patients. Performance on a wide range of motor tasks (including the Unified Parkinson Rating Scale and other measures) was monitored throughout the day at approximately monthly intervals for at least 4 months before, and up to 24 months after, transplantation. Patients were monitored with their routine medication or, on alternate sessions, after overnight withdrawal of medication. Ventral mesencephalic tissue from fragments of 5-8 week gestational age fetuses was collected and refrigerated during virological and bacteriological testing; a cell suspension of pooled, uncontaminated tissue was infected stereotaxically along multiple trajectories, computed according to CT scan, in the putamen contralateral to the more severely affected side. Patients received cyclosporin for the first 6 postoperative months. Fluorodopa PET scan indicates a progressive increase in striatal DOPA uptake, restricted to the operated striatum, at least 1 year postoperatively. Some, but not all, patients showed modest functional improvements on objective measures, beginning 2 months postoperatively and continuing for a period of months.

\section{K6.}

\section{Paroxysmal Ataxia and Paroxysmal Dystonia in Twins With Bilateral Hypodensities in the Basal Ganglia}

J. Muhunthan, E. Ives and T. Curran (St. John's, Newfoundland; Vernon, British Columbia)

Familial paroxysmal ataxia (FPA) is a rare and heterogenous condition that usually follows a autosomal dominant (idiopathic) or less frequently an autosomal recessive (? inborn metabolic error) pattern of inheritance. The ictal event is variable but classifically there is a sudden onset of gait ataxia and nystagmus, interictally mild abnormalities are usually found. FPA is known 
to be associated with neuromytonia and spinocerebellar degeneration. There is one single case report of association with paroxysmal kinesigenic choreathetosis.

We report 33 year old male twins who have had paroxysmal ataxia and dystonia with severe diaphoresis since the age of three. Interictally there is mild-moderate progressive generalized dystonia, nystagmus and a characteristic strained voice. Laboratory investigations reveal lactic acidosis and hyperalaninemia suggesting a defect in pyruvate metabolism. A muscle biopsy supported this diagnosis and biochemical studies of cultured fibroblasts are pending. The CAT scan of one patient shows significant hypodense lesions in the basal ganglia. The MRI is abnormal in both patients revealing hypointense signal abnormalities in the basal ganglia (mostly globus pallidus) on $\mathrm{T} 1$ images and a hyperintense signal on the $\mathrm{T} 2$ images. These findings suggest the diagnosis of adult Leigh's disease.

We will review the protein manifestations of defective pyruvate metabolism ("Leigh's disease") and the significance of paroxysmal ataxia and dystonia occurring in the same patient.

\section{K7.}

\section{Acute Neuroleptic-Induced Dystonic Reactions Have a Circadian Pattern}

\section{P. Rosebush and M. MAZUReK (Hamilton, Ontario)}

Acute dystonia is a well-recognized and common side-effect of neuroleptic medication. It has been proposed that the timing of acute dystonia reflects falling neuroleptic levels with an attendant exposure of postsynaptic receptors to a transient increase in presynaptic dopamine turnover, thereby creating a hyperdopaminergic state. According to this hypothesis, there should be no diurnal variation in the occurrence of acute dystonia in patients on a twice daily medication schedule. We have been examining this issue as part of a five year prospective study of consecutive patients taking neuroleptic medications for the first time on an acute care psychiatric inpatient service. Of the 178 patients thus far studied and treated with a twice daily neuroleptic dosing schedule (11:00 and 22:00), 75 (42\%) developed dystonia. Fully $86 \%$ of these reactions occurred between the hours of $11: 00$ and $22: 00$, while only $14 \%$ occurred between $22: 00$ and 11:00. This striking diurnal variation was not accounted for by the use of benzodiazepines. When we looked at the three hour period immediately prior to both the 11:00 and 22:00 doses, during which time all patients were awake, we found that there were 40 dystonic reactions prior to the 22:00 dose and only 2 dystonic reactions prior to the 11:00 dose. These data would suggest that circadian rhythms may play a role in determining the time of occurrence of acute dystonic reactions.
K8.

Pet Scan Studies in a Primate Model of Striatal Degeneration: Implications for the Mitochondrial Hypothesis of Huntington's Disease

M.F. Mazurek, E.S. Garnett, C. Nahmias, S. Furtado, A.Talalla, S. Garside, J. Woulfe and P. Rosebush (Hamilton, Ontario; Calgary, Alberta)

Positron emission tomography (PET) scan studies of patients with Huntington's disease consistently show reduced fluorodeoxyglucose (FDG) activity in the striatum, with preserved striatal fluorodopa (F-dopa) uptake. It has been generally assumed that the reduced FDG activity probably reflects the striatal degeneration associated with HD. We studied this issue in two female monkeys. Each animal received injections of saline at 10 stereotactic coordinates in the right striatum, and injections of the excitotoxin quinolinic acid (QUIN) in the left striatum. PET scans two weeks later showed symmetric FDG activity and F-dopa uptake in the striatum. Repeat PET scans 4 years later showed symmetric FDG activity, but reduced F-dopa uptake in the lesioned striatum of both animals. At autopsy, the monkeys had gross atrophy of the lesioned (left) striatum. These results indicate that:

(1) striatal degeneration does not, but itself, affect FDG activity, consistent with the hypothesis that the reduced striatal FDG activity observed in HD may reflect a primary failure of energy metabolism, perhaps arising from defective mitochondrial function;

(2) Long-standing excito-toxic lesions of the striatum can result in impaired dopaminergic function in the ipsilateral nigrostriatal system.

\section{K9.}

Migraine Headaches Ipsilateral to Focal, Non-Progressive Cerebral Lesions

\section{A.O. OGunYeml (St. John's Newfoundland)}

Headache during attacks of migraine are often unilateral or exhibit unilateral predominance. For some physicians, the coincident presence of headache with neurological deficits and abnormal neuroimaging tests (CT, MRI scans) may be grounds to disregard the diagnosis of migraine in patients who otherwise have the typical symptoms of the condition. We present 5 patients who experience migraine with their headaches always ipsilateral to the side of the cerebral lesions.

There are 3 female and 2 male patients with age-range of 20 years to 65 years. They have all been followed at our institution for 10 years or longer and have focal neurological deficits correlating with CT, MRI or both. Over the years, the clinical and neuroimaging findings have remained unchanged.

Each one of the patients has had multiple attacks of migraine without aura. The attacks responded well to anti-migraine medications. Four of them have partial epilepsy and have experienced post-ictal headaches, similar to those of their migraine.

We conclude that (1) migraine may occur in patients with non-progressive cerebral lesions with the headache consistently lateralized to the sides of the lesions and (2) the attacks respond favourably to anti-migraine medications. 
K10.

Migraine with Prolonged Aura: Correlation of Clinical and EEG Features

\section{A.O. Ogunyemi (St. John's, Newfoundland)}

The International Headache Classification Committee define migraine with prolonged aura as: "migraine with one or more aura symptoms lasting more than 60 minutes and less than a week; neuroimaging tests are normal". No laboratory tests were proposed to verify or objectively document the neurological deficits. We here, report five patients who had EEG recordings during migraine with prolonged aura. Follow-up serial EEG recordings were also obtained.

Four patients were female and one male. Age - range was 21 years to 31 years. During the attacks, the neurological signs consisted of: left hemianopia, tactile extinction and astereognosis (1 patient); nonfluent aphasia (2 patients); fluent aphasia with right hemianopia (1 patient); global aphasia and amnesia (1 patient). The EEG changes consisted of: polymorphic delta slow waves over the right hemisphere ( 1 patient); intermittent trains of theta and delta slow waves in left anterior temporal region (2 patients); polymorphic delta slow waves in left central - parietal region (1 patient); intermittent trains of delta slow waves over the left anterior temporal and bifrontal regions (1 patient).

Mild EEG abnormalities remained after resolution of the neurological deficits and headache. The EEG became normal within 2 weeks. The possible mechanisms of the EEG changes will be discussed.

K11.

Glossopharyngeal Neuralgia: Difficult Diagnosis, Easy Treatment

\section{R. BEnabou, P. Molina-Negro and J. HaRdy (Montreal, Quebec)}

Glossopharyngeal Neuralgia (GN) is a rare, and sometimes, underdiagnosed entity characterized by paroxysms of lancinating pain in the pharynx and tonsils, frequently irradiated to the ear and neck, typically triggered and worsened by activities such as swallowing, chewing and speaking.

The differentials and final diagnosis will be discussed based on 3 premises: Especific characterization of the pain, Research of functional deficits of the $I X^{\text {th }}$ cranial nerve and Electrophysiological studies of blinking reflexes.

We have studied 26 cases: 10 Secondary GN (SGN) -8 to cancer, 1 to herpes zona, 1 to trauma - and 16 Primary GN (PGN).

The patients with SGN were treated according to their primary pathology and 8 patients presenting PGN, in which medical therapy failed to control the pain, were submitted to Selective Sensory Rhizotomy of the $\mathrm{IX}^{\text {th }}$ cranial nerve in the posterior fossa.

All remain painfree after a post-operative follow up of 1 to 7 years, and do not complain of sensory deficits either because they are minor or because they were already present before surgery.

\section{NEUROPHYSIOLOGY}

L1.

Autosomal Dominant Myoclonus with Giant Somatosensory Evoked Responses in a Western Canadian Kindred with Dramatic Therapeutic Response to Clonazepam

A. KIRK (Saskatoon, Saskatchewan)

A Saskatchewan family of Ukrainian origin is reported in which several members in three generations, now scattered across the four westernmost Canadian provinces, presented with myoclonus. To date seven individuals in three generations have been examined, five of whom have reproducible extremely high voltage cortical potentials and clinical myoclonus evoked by somatosensory stimulation. Two individuals were asymptomatic and had normal amplitude evoked potentials. The youngest symptomatic member was 23 , the oldest his 78-year-old grandfather. Patients reported very slowly worsening myoclonus usually evoked by sudden unexpected stimuli to a limb. Early in the course, myoclonus tended to affect one limb at a time and was no more than a nuisance but the eldest affected family member had suffered numerous injuries from falls due to generalized myoclonus. He had obvious spontaneous multifocal and generalized myoclonus. Examination showed no other neurologic deficits in any family members. There was no history of seizures. EEG showed no epileptiform activity and MRI was normal in one affected 51-year-old woman. Two family members showed dramatic clinical improvement on a low dose of clonazepam with cessation of falls in the eldest.

\section{L2.}

\section{Monitoring of Motor Tracts with Spinal Cord Stimulation}

\section{S.S. HaGhighi, R.W. Gaines and J.J. ORo (Columbia, Missouri)}

We recorded motor evoked potentials by epidural electrical stimulation of the spinal cord in cats $(n=22)$. At lower electrical intensities $(<5 \mathrm{~V})$, the posterior column sensory fibers were antidromically activated. These responses were recorded from the lumbar spinal cord $(n=22)$, dorsal root $(n=80)$, and peroneal nerve $(n=40)$. Spinal evoked response resisted lesioning and showed a minimal change after a spinal cord hemisection. Dorsal rhizotomy abolished the ipsilateral peroneal nerve action potential, indicating antidromic activation of afferent fibers.

Motor tract activation was accomplished by recording the ventral root $(n=40)$ and muscle action potential $(n=10)$ using single pulse stimulation $(>50 \mathrm{~V})$. These responses did not change after the dorsal rhizotomy suggesting involvement of non-sensory pathways. Point-to-point spinal cord stimulation revealed the dorsal and dorsalateral sites to evoke the highest amplitude muscle action potentials.

These findings indicate that spinal cord stimulation activates both sensory and motor tracts which can be recorded at various sites along the central or the peripheral nervous system. 
L3.

\section{Reciprocal Inhibition in Hemiplegic Patients with Special Reference to Functional Recovery and Spasticity}

\section{Y. OKumA and R.G. LeE (Calgary, Alberta)}

Reciprocal inhibition between ankle flexors (TA) and extensors (soleus) was studied in 12 hemiplegic patients and 23 control subjects in the resting state. The amount of disynaptic Ia inhibition was determined from the short latency suppression of the soleus or TA H-reflexes by conditioning stimulation of the antagonistic muscle nerves. Ia inhibition to the soleus motoneurones was increased in patients who showed a good recovery of function with mild spasticity, but was not changed in the poor recovery patients with more marked spasticity. Serial recordings from the same patients showed a significant increase in Ia inhibition during the period of recovery following stroke. The late (D1) inhibition, presumably due to presynaptic inhibition, was deceased in the patients, although correlations between the amount of inhibition and the symptoms were rather unclear. These findings suggest that changes in excitability of the la inhibitory pathways are closely related to some of the clinical features of hemiplegia.

\section{L4.}

\section{"Anterior Slow Alpha of Drowsiness" EEG Patterns do not Represent the Alpha Rhythm}

R.J. Broughton, J. Hasan and W. Dunham (Tempere, Finland; Ottawa, Canada)

It is often stated that the parieto-occipital alpha rhythm slows and diffuses anteriorly in drowsiness prior to stage 1 sleep patterns (mixed frequency mainly theta activity). We investigated by topographic EEG (Bio-Logic Brainscan) and source dipole analysis (Scherg software) both the anterior alpha in drowsiness and the alpha rhythm across sleep onset in 20 patients (mean age 38.9, range 17-77) referred for a diagnostic EEG or multiple sleep latency test. 21 EEG channels (10/20 system) were recorded and the EEGs were essentially normal.

Results: "Anterior slow alpha of drowsiness" rarely represented slowing of the alpha rhythm. Almost always it consisted of a separate EEG event which often co-existed with, but varied independently from, the alpha rhythm. It was maximum in the midline centro-frontal region (vs. parieto-occipital), slower in frequency (by 1.0-2.0 Hz), dependent on a different state (drowsiness vs. wakefulness), and had both a different reactivity and a separate equivalent source dipole (near the thalamus vs. mesial occipital lobes). It disappeared in NREM and REM sleep and seemed to be specific to sleep onset.

L5.

Interactions Between the Eye and Hand Motor Systems: Disruptions Due to Cerebellar Dysfunction

\section{R.G. LEE and P. vaN DONKELAAR (Calgary, Alberta)}

Seven subjects with cerebellar dysfunction and seven controls performed arm movements to track or intercept a moving visual target under the following conditions: 1) with full vision of the hand and unrestricted eye movements, 2) while visually fixating a stationary target, and 3) without vision of the hand. Some of the irregularities of hand trajectories for the cerebellar subjects were clearly related to the presence of saccadic intrusions within the smooth pursuit eye movements. When eye movements were restricted by having the subjects visually fixate, arm movements showed less variability. Restricting vision of the hand also resulted in smoother limb movements as well as a reduction in the number of saccadic intrusions.

The quality of eye movements was also influenced by the accuracy of simultaneously produced limb movements. In the control subjects eye movements became more accurate when the hand was also used to track the target whereas smooth pursuit responses for the cerebellar subjects deteriorated in this condition. Thus there appears to be a two-way interaction between the motor control systems for the eyes and the limbs. Disruption of one system may adversely affect performance with the other, and this may account for some of the difficulty which cerebellar subjects have with tasks that require combined eye and hand movements.

\section{L6.}

\section{Decrease of Myotonin-Protein Kinase Activity in Myotonic Dystrophy}

J.-P. Bouchard, P. Etonguay-Mayer, R. Faure and J. PUYMIRAT (Quebec, Quebec)

The molecular basis of Myotonic dystrophy (DM) is known to include a mutational expansion of a repetitive tri-nucleotide sequence (CTG)n located in the 3' untranslated region of mRNA from a gene encoding a putative serine-threonine protein kinase product (M-PK). Using a specific polyclonal antibody against a peptide sequence of the predicted gene product, we have identified a $54 \mathrm{kDa}$ protein in human skeletal muscle. This protein has a tyrosine kinase activity in skeletal muscle suggesting that M-PK is a protein kinase with dual specificity. In the adult form of DM, the levels of M-PK mRNAs remain unchanged (41 \pm 6 relative IOD in control, $\mathrm{n}=6 ; 41.9 \pm 5$ relative IOD in DM, $\mathrm{n}=6$ ), as determined by dot blot analysis. Furthermore, the levels of M-PK activity are decreased by $42 \%$ in skeletal muscle of DM $(61.728 \pm 2542$ to $36.000 \pm 2755 \mathrm{cpm}$ of phosphorus transferred $/ 10 \mathrm{~min} / 60 \mu \mathrm{g}$ of total protein in control and DM, $\mathrm{n}=6$ ).

It is the first report showing a decrease of M-PK activity in the muscle of DM. This strongly suggests a major role of M-PK in the pathophysiology of DM.

\section{L7.}

\section{Sulindac in Experimental Diabetic Neuropathy}

P. VAN DER SLOOT and D.W. ZochODNE (Calgary, Alberta)

In two previous studies we have demonstrated prevention of electrophysiological abnormalities in experimental STZ-induced diabetic neuropathy (EDN) of rats using nonsteroidal antiinflammatory agents: indomethacin and sulindac. Sulindac might benefit EDN because it inhibits both cyclo-oxygenase and aldose reductase. In this work, we examined whether 1 month of 
sulindac treatment reversed or improved established EDN (STZ given as $65 \mathrm{mg} / \mathrm{kg}$ ip in 8 week old male Spraque-Dawley rats) of 3 months duration. Sulindac-treated diabetic rats $(6.0 \mathrm{mg} / \mathrm{kg}$ $5 / 7$ days weekly by gavage) were compared to untreated diabetics, nondiabetic controls and sulindac treated control rats. Diabetic rats developed slowing of conduction velocity in caudal sensory (CSCV), sural sensory (SSCV), caudal motor (CMCV) and sciatic tibial motor (STCV) fibers. Sulindac improved CMCV and, to a less extent SSCV but there was only a nonsignificant trend toward higher CSCV and there was no improvement in STCV. There was an improvement in the amplitude of the compound "M" potential from caudal motor fibers. Sulindac provided modest improvement in some indices of neuropathy in this reversal study, but there was less efficacy than in the preventative study. Earlier or longer therapy duration is likely required to demonstrate more widespread benefit.

\section{L8.}

\section{CGRP and the Response of Peripheral Nerve to Injury}

D.W. Zochodne, J.A. Racicot, K. Hargreaves, L.T. Ho and K.A. Sharkey (Calgary, Alberta; Minneapolis, Minnesota; Kingston, Ontario)

Calcitonin gene-related peptide (CGRP) is a widely distributed neuropeptide and potent vasodilator. We studied the presence and action of CGRP in experimental sciatic neuromas of rats. Neuromas were created by resection of the distal sciatic nerve and its branches with the proximal stump allowed to retract above the site of the incision. Local perfusion was examined by measuring hydrogen clearance using microelectrodes inserted within the neuroma, and by laser doppler flowmetry, measuring $\mathrm{RBC}$ flux of the extrinsic vascular plexus of the neuroma. Both techniques detected post-injury hyperemia, maximum at $48 \mathrm{hrs}$ but persistent at 14 days and not observed in sham-exposed nerves or sciatic nerves contralateral to injury. At $48 \mathrm{hrs}$ (and to a lesser extent at $24 \mathrm{hrs}$ ), topical hCGRP (8-37) at $10^{-4} \mathrm{M}$, a CGRP receptor antagonist, reversed the hyperemia. Within the neuromas, there was intense, diffuse and globular CGRP immunoreactivity at $48 \mathrm{hrs}$ arising from CGRP accumulation in swollen axons or the extracellular space. CGRP accumulation at $48 \mathrm{hrs}$ (351 fmol/mg neuroma tissue vs. $77 \mathrm{fmol} / \mathrm{mg}$ in noninjured contralateral sciatic nerve; $p=0.005$ ) was confirmed by radioimmunoassay. CGRP accumulates in a time-related fashion within experimental neuromas where it induces prominent local vasodilation.

L9.

Local Superfusion Studies of Morphine and Spantide Over Experimental Neuromas

D.W. Zochodne and L.T. Ho (Calgary, Alberta; Kingston, Ontario)

Local opiate superfusion of injured tissues, for example postoperative knee joints, has been advocated as an avenue of pain therapy. It is not certain, however, whether local opiates would adversely alter local injury and repair processes. We investigated the influence of local superfusion on the development of injury hyperemia in 14 day old sciatic neuromas of Sprague-Dawley rats. Neuromas were created by resection of the distal sciatic nerve and its branches. In separate rats, we used implantable mini-osmotic pumps to superfuse the following solutions over the site of the sciatic nerve resection for 14 days during neuroma development: normal saline, spantide (substance $\mathrm{P}$ antagonist and vasoconstrictor), morphine. At endpoint, the neuromas were exposed and local blood flow was measured by hydrogen clearance before and after topical application of a CGRP receptor antagonist. Local blood flow was higher than control values, but did not differ significantly from values in non-perfused neuromas irrespective of the superfusion solution. Superfusion did not confer sensitivity to the CGRP antagonist. Morphine and spantide might influence local blood flow at early time points in neuroma development (when CGRP action is prominent) but chronic superfusion did not adversely influence later injury hyperemia.

\section{L10.}

Cerebellar Evoked Potentials for Monitoring Spinal Cord Function during Neurosurgery: Results of a Prospective Controlled Trial

M.G. Fehlings, M. Moncada, F. Gentili, P. Manninen and J. Hulbert (Toronto, Ontario)

Intra-operative somatosensory evoked potentials (SSEPs) can be of limited usefulness in predicting spinal cord injury because they are transmitted primarily through the dorsal columns and therefore do not reflect integrity of ventral tracts. We have recently shown in animal experiments that evoked potentials (EPs) recorded from the posterior fossa or cerebellum directly (CEP) after posterior tibial nerve (PTN) stimulation reflect the integrity of ventral tracts. In the present study we prospectively evaluated the CEP in comparison with the SSEP in a controlled cohort of patients undergoing spinal decompressive or skull base procedures ( $n=46$; age range 18-82). EPs were recorded transcranially from the posterior fossa (CEP) or from the vertex (SSEP) after PTN stimulation $(4.72 \mathrm{~Hz} ; 250 \mu \mathrm{s}$; bandpass $30-$ $3000 \mathrm{~Hz}$ ). Reliable CEPs were recorded in all but one patient who has a severe cervical myelopathy with a major motor deficit. In one patient with impaired dorsal column function but relatively preserved motor function, a SSEP could not be obtained whereas reliable CEPs were obtained. The peaks (mean latency \pm SD) of the SSEP (P37: $41.6 \pm 5.2 \mathrm{~ms}$; N45 49.8 $\pm 5.4 \mathrm{~ms}$ ) varied independently ( $\mathrm{p}>0.05$; MANOVA) of the peaks of the CEP (N33: $31.5 \pm 2.8$; P40: $36.6 \pm 3.59$ ). Our results suggest that the CEP is a novel EP modality which appears to reflect the integrity of ventral spinal cord tracts and may provide complementary neurophysiological information to conventional SSEPs. 


\section{Poster Presentations}

\section{SESSION I}

\section{I-1. PEDIATRIC EPILEPSY}

P1.

\section{Location of Epileptic Foci on Interictal and Postictal Single Photon Emission Tomography in Children with Localization-Related Epilepsy}

H. Otsubo, P.A. Hwang, C. Li, D. Gilday, S. Blaser, L.E. BECKER, V. JAY and H.J. HoFFMAN (Toronto, Ontario)

A retrospective analysis of single photon emission tomography (SPECT) studies, interictal alone and combined interictal and immediate postictal, in children with localization-related epilepsy who underwent epilepsy surgery. Since location of abnormal cerebral perfusion has been demonstrated by SPECT using $99 \mathrm{~m}$-technesium hexamethylpropylene amine oxime, combined interictal and postictal SPECT studies have been reported to correlate favorably with localization-related epilepsy.

Fifty five children with locarization-related seizures were studied with video-EEG telemetry under epilepsy monitoring unit (EMU), CT, MRI and SPECT, between 1987 and March 1993. The interictal SPECT was performed in 55 patients, and immediate postictal SPECT was studied for 17 in EMU. Diagnostic accuracy using combined interictal and postical SPECT scans; 15 of $17(88 \%)$, was significantly better than interictal scans alone; 21 of 38 (55\%), for abnormal perfusion corresponding to EEG abnormality $\left(c^{2}=5.647, p=0.0175\right)$. There is good correlation between EEG localized epileptogenic focus and hypoperfusion in the temporal lobe epilepsy rather than extratemporal. Particularly mesial temporal sclerosis showed localized abnormal perfusion, dual pathology did not show such correspondence to EEG abnormality. This cerebral perfusion on interictal and postictal SPECT can improve localization of epileptic focus for epilepsy surgery.

P2.

Psychosocial Adaptation of Adolescents with Epilepsy: Selfand Parent-Perceptions

M.J. Pelletier, I.G. Manion, S. Whiting, D. Keene, P. Jacob, P. HuMPhreYs and A.WATTERS (Ottawa, Ontario)

Research suggests that epilepsy can have deleterious effects on all aspects of psychosocial functioning across the lifespan. The purpose of this study was to compare the psychosocial adaptation of adolescents with epilepsy to that of healthy adolescents. A matched case-control design was used to compare psychosocial functioning in $\mathbf{4 6}$ adolescents with epilepsy (ages $11-18$ ) and in 46 controls. Self- and parent-reports of adolescent behavioural, emotional and social functioning (i.e., Youth SelfReport, Child Behavior Checklist) assessed the impact of epilepsy on adolescent perceived psychosocial adjustment.

Adolescents with epilepsy viewed themselves as having greater problems with social competence compared to controls, $t(87)=-3.31, p<.001$, with a greater proportion of epileptics scoring in the borderline and clinical ranges. Their parents reported even greater differences in social competence with
$30.4 \%$ of cases vs. $0 \%$ of controls scoring in the clinical range. Parents also identified more emotional difficulties in the epileptics compared to controls, $t(90)=2.25, p<.05$. Differences in social-emotional functioning were not explained by medication use or level of seizure control. Adolescents with epilepsy were more likely than controls to have failed a grade $(26.1 \%$ vs. $6.5 \%$ ), and to be in a special class or receiving resource help $(21.7 \%$ vs. $2.2 \%)$. These results underscore the impact of epilepsy in adolescence, particularly in the areas of social competence, academic success, and emotional well-being, with implications for both prevention and intervention.

P3.

\section{Evolution of Tumour Following Remission of Intractable} Seizures

J. Hukin, M.B. Connolly and K. FarRell (Vancouver, British Columbia; Boston, Massachusetts)

Complete seizure control and remission following antiepileptic drug withdrawal is rare in patients with brain tumours.

The patient presented with right partial and secondarily generalised seizures which occurred frequently between 3 and 6 years of age despite treatment with carbamazepine, valproate and phenytoin. A CT head scan at age 34 months demonstrated a left parietal hypodense lesion, which measured $2 \mathrm{~cm}$ in diameter. Six CT head scans repeated over the subsequent 6 years demonstrated no change. Following the addition of phenobarbital at 6 years of age, the seizures were controlled for 9 years and anticonvulsant therapy was discontinued at 15 years of age. The patient was well on no medication for 13 months after which time he developed headaches. Examination demonstrated papilledema and a right hemiparesis. CT head scan demonstrated a large enhancing left posterior parietal cystic lesion with midline shift. A grade IV malignant parietal astrocytoma was removed at 17 years of age.

The seizures in this patient were difficult to control initially. Because of lack of change in the CT findings over a period of 6 years together with eventual seizure control, the hypodense lesion was considered to be an infarct. The absence of seizures after the medication withdrawal demonstrates the fluctuation in epileptogenicity which may be associated with brain tumors.

\section{P4.}

Benign Rolandic Epilepsy - Atypical Clinical Features Are Very Common

E.C. Wirrell, P. Camfield, C. Camfield, J. Dooley and K. GoRDON (Halifax, Nova Scotia)

Objective: To determine the frequency of atypical clinical and electrographic features in children with Benign Rolandic epilepsy (BRE).

Design: Retrospective case series.

Setting: Tertiary care pediatric hospital.

Methodology: 42 children with BRE were seen through our Neurology Department between 01/01/91 and 31/12/93. Their charts were reviewed for atypical clinical features, imaging studies and results, total number of seizures at initial presentation and last followup and use of anticonvulsants. Atypical clinical features included status, developmental delay, daytime only 
seizures, screaming as a seizure component and post-ictal Todd's. All children had at least one EEG and these records were reviewed for atypical electrographic features such as unusual location, atypical spike morphology or abnormal background.

Results: Atypical clinical features were seen in 50\% and atypical electrographic features in $31 \%$. CT scans were performed in 15 patients and were consistently normal. Treatment with anticonvulsant medication was initiated in $40 \%$. Although patients with atypical features did not have an increased seizure frequency, they were more likely to undergo imaging studies $(p<.01)$ and to be commenced on anticonvulsant medication $(p<.02)$.

Conclusions: Our experience suggests that atypical clinical and electrographic features are the rule rather than the exception in BRE. Further work must be done on development of a reliable definition of this common entity.

\section{P5.}

\section{Lamotrigine Used in Pediatric Patients at The Children's Hospital in Eastern Ontario}

\section{S.E. Whiting, D. KeEne and P. Humphreys (Ottawa, Ontario)}

Lamotrigine is a novel antiepileptic drug currently being evaluated in Pediatrics.

31 patients ages 1 to 19 years (mean 8 years) were treated with Lamotrigine as add on medication. Patient response was analyzed based on seizure type. Partial seizures occurred in 15, atonic 13 , atypical absence 11 , tonic 5 , generalized tonic clonic 3 and myoclonic 2. Lamotrigine was used alone in 2 patients but $74 \%$ of patients had at least 2 drugs at the time of use. Dosage ranged from 25 to $400 \mathrm{mg}$ with final dosage based on weight being 1.8 to $10 \mathrm{mg} / \mathrm{kg}$ (mean $4.9 \mathrm{mg} / \mathrm{kg}$ ). There was no difference in dosage in successes versus failures.

Success was defined as $50 \%$ decrease in a seizure type. Patients with atonic seizures and atypical absence showed the best response, 11 of 13 and 10 of 11 respectively. 6 patients became completely seizure free (atypical absence 3, atonic 3). Only 4 of 15 patients with complex partial seizures showed a response.

Side effects were rashes 5 , behaviour changes 3 , increased seizures 2.

Lamotrigine appears to be a useful anticonvulsant in generalized seizures especially atypical absence and atonic seizures.

P6.

\section{Absence Status Epilepticus (ASE) Presenting as Complex Partial Seizures in Childhood}

\section{A.N. Prasad and M.H. Libenson (Boston, Massachusetts)}

ASE is an uncommon diagnosis in childhood. We present the case of an intelligent 9-year-old boy who presented with spells of altered responsiveness. The first episode consisted of unresponsiveness and wandering about at a museum and falling asleep on the ride home. A second spell occurred during an ice hockey toumament when, as goaltender, he began skating slowly around the hockey rink with eyes at "half mast". The third episode occurred at the end of a baseball game when he did not respond to his parents, walked aimlessly about repetitively striking the ground with a baseball bat, and then became agitated and incontinent of urine. The EEG showed a photoparoxysmal response and $1.5-5 \mathrm{~Hz}$ generalized spike wave discharges with anterior predominance, one such discharge accompanied by eye fluttering. He remained seizure-free on sodium divalproex for two years and medication was discontinued. Five months later, he presented with 3 hours of confusion, slurred speech, inconsistent response to questions, and intermittent forced upgaze; the ER physician felt he was "postictal". The EEG showed continuous, generalized 1-3 Hz spike wave discharges. The EEG and mental status both normalized promptly with intravenous diazepam. ASE is an unusual initial manifestation of primary generalized epilepsy in childhood and should be considered in the differential diagnosis of confusional states in children.

P7.

\section{Psuedoseizures Manifesting as Status Epilepticus}

J. Hukin, K. Farrell, M. Connolly and A. Hill (Vancouver, British Columbia)

Pseudoseizures often present a diagnostic dilemma because of their protean manifestations. We report a patient with pseudoseizures presenting as status epilepticus.

The patient was a 13-year-old female with borderline intelligence. She had an occipital encephalocele and hydrocephalus treated with a right ventriculoperitoneal shunt. Partial seizures with and without secondary generalization which had begun at age 6 months occurred infrequently on carbamazepine. At 12 years, she presented in apparent status epilepticus which did not respond to intravenous phenytoin, phenobarbital, diazepam and paraldehyde. the shunt catheter was disconnected and revised. The apparent status epilepticus recurred postoperatively and the child was reintubated. Following extubation the next day, the episodes recurred and suspicion arose that they might represent pseudoseizures. Video-EEG demonstrated five episodes without associated ictal EEG correlate. A pediatric psychiatrist was consulted and demonstrated definite psychopathology. Phenytoin and phenobarbital were discontinued. The episodes ceased after 3 days and have not recurred. The seizures have been controlled satisfactorily on carbamazepine.

This case demonstrates the unusual presentation of pseudoseizures as status epilepticus, an observation which had not been reported previously to our knowledge.

P8.

Shuddering Attacks Mimicking Infantile Spasms "Consider Shudder not Spasm a Benign Outcome"

D.J. Buckley, M.S. Hall and S. Penney (St. John's Newfoundland)

We present our experience of shuddering attacks at the Janeway Child Health Care Centre, St. John's, Newfoundland. Five infants were referred to the Neurology Division for assessment of probable infantile spasms over a period betwee 1989 to 1994 , ranging in ages 7 to 13 months.

The episodes consisted of sudden unexplained motor activity that occurred paroxysmally and which interfered with the child's activity. Some of the events occurred repeatedly. 
All infants remained well and did not deteriorate.

Video EEG Monitoring or routine EEG Monitoring did not reveal epileptiform activity in association with these events.

No association with benign essential tremor was found in all our cases.

These infants have been followed up to 5 years of age and continue to be normal, thus confirming a benign outcome.

Shuddering attacks can occur in the same age group as children with infantile spasms. It is very concerning that both have similar clinical features. Correct diagnosis is essential to give the excellent prognosis these children have.

P9.

\section{Quantitative EEG Analysis in Premature Infants}

E.A. MacDonald, D.G. Brunet and J.S. Lawson (Kingston, Ontario)

Power from 12 electrodes in 77 recordings in premature infants between 30 and 37 1/2 weeks post conception was analysed and a correlation matrix derived and subjected to principle components analysis.

Examination of the correlation patterns among output from 12 electrodes suggested averaging the power output from adjacent electrodes; the four anterior left electrodes (left anterior), the four anterior right electrodes (right anterior), the two posterior left electrodes (left posterior), and the two posterior right electrodes (right posterior).

In general, power declined with increasing frequency. Different power output according to location (anteriority laterality) tended to occur in the higher frequency band $(10-20 \mathrm{~Hz})$ and this was more marked in the brain damaged infants. Power tended to decline with increased gestation of age for both groups, however, this decline was present only in the highest frequency band and was most marked in the brain damaged infants.

\section{I-2. PEDIATRIC GENERAL}

P10.

Hydrocephalus and Mineralizing Intracranial Angiopathy in Association with Hypercholesterolemia and Elevated Lipoprotein(a) Levels

A.Y. Manzur, J. Frohlich, K. Poskitt, J. Tibbles, M.G. NORMAN and J.U.C. CRICHTON (Vancouver, British Columbia)

High blood levels of lipoprotein(a) ( $L p(a))$ are associated with increased risk of cerebrovascular disease. We report a boy with hydrocephalus and mineralizing intracranial angiopathy who had hypercholesterolemia and high $\mathrm{Lp}$ (a) levels.

After developing normally, he developed strabismus, irritability and vomiting at 20 months. He had communicating hydrocephalus which required shunting. By 3 years he developed autistic features and seizures.

CT (4 and 5 years of age) demonstrated progressive perivascular calcifications. MR scan demonstrated multiple foci of increased signal (T2) in grey and white matter in cerebellum, temporal lobes, basal ganglia and corpus callosum. Abnormal meningeal enhancement was noted in basal cisterns after contrast injection. Cerebral angiography was normal. CSF had no cells but protein levels were high and CSF/serum albumin ratio was increased. IgG synthesis index was normal. Viral and bacterial serology was negative. Temporal lobe biopsy revealed thickened arachnoid, abnormal vessels, leptomeningeal fibrosis and focal gliosis of cerebral cortex. Plasma $\mathrm{Lp}$ (a) $(3608 \mathrm{mg} / \mathrm{L})$ and cholesterol $(7.15 \mathrm{mmol} / \mathrm{L})$ levels were elevated. Investigations for vasculitic, metabolic, clotting and infectious disorders were negative.

These observations report an unusual association of lipid abnormalities with hydrocephalus and mineralizing angiopathy.

\section{P11.}

\section{Pediatric Idiopathic Intracranial Hypertension: an Infectious Etiology?}

\section{K.E. GoRDOn (Halifax, Nova Scotia)}

Objective: To determine whether cases of Pediatric Idiopathic Intracranial Hypertension present seasonally or in clusters.

Design: Retrospective case series.

Setting: Tertiary care pediatric hospital.

Patients: Health records identified 46 children with ICD9 code 348.2 (Benign Intracranial Hypertension) presenting between $79 / 04 / 01$ and 93/03/31. After chart review, 28 cases were identified which satisfied the modified Dandy's diagnostic criteria for Idiopathic Intracranial Hypertension.

Analysis: The months of symptom onset were plotted to produce a frequency histogram. The number of cases in two month intervals were examined for seasonal presentation and clustering using a chi-square analysis.

Results: The 28 cases ranged in age from 3-15 years (median 12 years), and had been symptomatic for $<1-16$ weeks (median 2 weeks). A significant seasonal presentation was demonstrated $\left(\mathrm{X}^{2} 5 \mathrm{df}, \mathrm{p}=.02\right)$, with symptom onset showing a major peak in January and a minor peak in mid August. Clustering of cases was demonstrated by significantly more periods with zero cases or 2 or more cases than would have been predicted using the poisson distribution ( $\mathrm{X}^{2} 3 \mathrm{df}, \mathrm{p}=.04$ ).

Conclusions: Clustering of cases with such a distinctive seasonal presentation suggests a potential infectious etiology for pediatric idiopathic intracranial hypertension.

\section{P12.}

\section{Progressive Axial Muscle Rigidity During Infancy: A New Entity}

A.Y. Manzur, R.D. Keyes, M. Bennett and E.H. Roland (Vancouver, British Columbia)

Continuous muscle contraction with respiratory failure in infancy occurs in hyperekplexia, Isaac's syndrome, paramyotonia congenita, Schwartz-Jampel syndrome and Z-band myopathy. We report clinical, EMG and histological features in an infant with progressive axial muscle rigidity which has not been reported previously.

This female infant of Dutch parents (first cousins) developed progressive rigidity of axial muscles at 1 month. Ventilation for respiratory failure was required at 4 months. By 9 months, muscle rigidity involved proximal limbs. Clonazepam, phenytoin, mexiletine, nifedipine, dantrolene and prednisone produced no improvement. 
The CPK was 3,369u (N:60-305u). The EKG, echocardiogram, motor and sensory nerve conductions were normal. EMG of paraspinal muscles at rest demonstrated continuous, repetitive firing of normal motor units. There were no myotonic discharges. Clinical and EMG abnormalities were unchaged by neuromuscular blockade with vecuronium. Muscle biopsy demonstrated non-specific type II fibre atrophy with normal histrochemistry and electronmicroscopy. Screening for the SCN4a (paramyotonia) gene was normal.

These observations suggest an autosomal recessive ion channel defect or abnormal calcium uptake by muscle sarcolemma associated with continuous repetitive depolarization of axial muscle fibres. Ion channel studies on cultured myoblasts are underway.

\section{P13.}

\section{Ophthalmologic Presentation in Pediatric Multiple Sclerosis}

M. Steinlin, S. Blaser, D.L. MacGregor and J.R. Buncic (Toronto, Ontario)

Children are known to have different initial manifestations of multiple sclerosis (MS). Eye problems were studied in a retrospective review of 17 children with definitive MS (Poser's criteria, 1983). Mean age of onset was 13.5 years. Follow up varied from less than 1 to 6 years. Of 16 children (94\%) with ophthalmologic symptoms $8(47 \%)$ presented with the initial problem in vision. 4 of the 12 children with optic neuritis (ON) had visual loss as the initial symptom of MS. In 3 of the 12 children episodes were recurrent. Progressive uveitis affected one child. Brainstem symptoms involved only 4 children (1 VI nerve palsy, 1 VI nerve palsy and internuclear ophthalmoplegia (INO) and 2 INO. Four children showed cerebellar signs (mystagmus, saccadic pursuit). In 3 other children localization was less specifically brainstem or cerebellum. Recovery from symptoms was complete or almost complete in most of the children. Neuroimaging was successful only in 1 child in confirming the localization of the lesion. Multiple evoked potential were helpful for investigation of ON, but less supportive specifically for brainstem lesions.

In conclusion ophthalmologic symptoms are very frequent in children with MS and should aid in reaching the diagnosis.

\section{P14.}

\section{Five " $N$ of 1" Trials of Melatonin in Children with} Intellectual Deficits and Fragmented Sleep

P.R. Camfield, K. Gordon and J. Dooley (Halifax, Nova Scotia)

Melatonin effectively controls some sleep disorders. Five children, ages 3-13 years, with mental retardation presented with severely fragmented sleep which was highly disruptive to family life and resistant to hypnotics and behavioral therapy. 3 had visual deficiency but preserved light-dark discrimination. Each child underwent a 10 week double blind trial of melatonin. Each week, the child received once daily melatonin at 6 PM $(0.5$ $\mathrm{mg}$ for 3 cases, $1.0 \mathrm{mg}$ for 2 ) or placebo in random order. Overall each child had 5 weeks of placebo and 5 weeks melatonin. During the melatonin weeks, there was no significant improvement in length of sleep, number or duration of night awakenings, or pattern of day sleep. We conclude that melatonin
0.5 or $1 \mathrm{mg}$ is ineffective in intellectually impaired children with fragmented sleep. Further trials with higher dose may be indicated.

\section{P15.}

Evolving Trends in the Incidence and Management of Post Hemorrhagic Hydrocephalus in Premature Infants (Birthweight $<1500 \mathrm{~g}$ )

A.Y. Manzur, B.A. Lupton, E.H. Roland, P. Steinbok and A. HILL (Vancouver, British Columbia)

Post hemorrhagic hydrocephalus (PHH) is a major complication of intraventricular hemorrhage (IVH) in premature newborns. We report evolving trends in incidence and management of PHH in the last decade.

We reviewed the records of all premature newborns $(<1500$ g) admitted to B.C.'s Children's Hospital (January, 1983 December, 1993). Criteria for diagnosis of $\mathrm{PHH}$ included rapid increase in head circumference or ventriculomegaly on cranial ultrasonography associated with clinical signs of raised intracranial pressure. The incidence of IVH (excluding germinal matrix hemorrhage alone) decreased from $20 \%$ to $10 \%$ whereas the incidence of $\mathrm{PHH}$ increased from $10 \%$ to $16 \%$ during the study period. 55 to 424 infants with IVH developed PHH. In 20, ventricular dilation arrested or resolved spontatneously. In 21/35 infants, a ventricular access reservoir was placed. $13 / 21$ required subsequent ventricular peritoneal (VP) shunt insertion. 14 infants received VP shunts without prior intermittent ventricular drainage. A total of 27 of the 55 infants with $\mathrm{PHH}$ required eventual shunt insertion. The incidence of VP shunt insertion for PHH has remained relatively constant.

These data demonstrate a decreasing incidence of IVH but increased incidence of $\mathrm{PHH}$ during the last decade. In addition, there is a definite role for the use of the ventricular access reservoir.

P16.

\section{Immunosuppressive Measles Encephalitis}

S.D. Levin, R. Chen, D. Ramsay, L. DeVeber and L. Assiss (London, Ontario)

A case of immunosuppressive measles encephalitis (IME) in a 12-year-old boy in remission from acute lymphoblastic leukaemia is described. He had been vaccinated against measles at 1 year and developed acute lymphoblastic leukaemia at 22 months which relapsed when he was 5 and 7 years old. He developed a generalized rash with fever which lasted for 5 days and serum measles IgM antibody was elevated indicating acute measles infection. 6 months later he sustained a brief generalized tonic-clonic seizure and after 4 days developed epilepsia partialis continua. He died 1 month later, 7 months after the acute measles infection. Repeated CSF analyses were normal. The EEG showed diffuse delta waves and periodic epileptiform discharges originating in the right occipital region. MRI showed areas of increased signal in the T2 weighted and proton density images. Histologic examination of the brain showed abudant intranuclear eosinophilic inclusions in neurons and oligodendroglia bilaterally in the deep cortial areas of the parietal and occipital lobes associated with marked astrocytosis and linear 
vacuolation of the underlying white matter. Electron microscopy demonstrated paramyxovirus nucleocapsids. IME is one presentation of measles inclusion body encephalitis. The diagnosis requires a high index of suspicion in immunosuppressed children. Definitive diagnosis requires biopsy from an involved site.

\section{P17.}

\section{Multiple Sclerosis in Childhood: The Role of Evoked} Potentials

\section{C.L. Pridmore, M.J. TAYlor and S. Blaser (Toronto, Ontario)}

We described the clinical and laboratory findings in 23 children with definite (17) or probable (6) multiple sclerosis (MS). Onset of symptoms referable to the disease was from 5 to 17 years. The most common initial clinical manifestations were weakness or incoordination (16), visual symptoms, including diplopia and optic neuritis (13) and sensory symptoms (9). Definite childhood MS was more frequent in girls (1.4: I) and often had a relapsing-remitting course.

At least one evoked potential (EP) modality was abnormal in $88 \%$ of definite cases. Visual evoked potentials (VEPs) showed the highest sensitivity and most frequently detected asymptomatic central nervous system (CNS) involvement. Magnetic resonance imaging (MRI) revealed lesions typical of MS in $80 \%$ of definite cases.

Supratentorial abnormalities were the most common and the majority of children had multiple white matter lesions. EP studies and MRI are sensitive diagnostic tools which can confirm equivocal clinical findings and reveal subclinical CNS involvement.

\section{P18.}

\section{Successful Treatment of Painful Crises in Fabry's Disease} with Low Dose Morphine

\section{K. Gordon, M. Ludman and A. Finley (Halifax, Nova Scotia)}

Fabry's disease is an X-linked disorder characterized in childhood by angiokeratoma, corneal opacities, and pain. Pain manifests as crises and/or continuous discomfort. Phenytoin, carbamazepine, and neurotropin, alone or in combination have been used as prophylactic therapy. Narcotic analgesics are "partially effective" (Desnick, The Metabolic Basis of Inherited Disease, 1989).

At age 7, our patient began experiencing an intermittent intense "burning" sensation within his feet and hands. Treatment with ASA, acetominophen, Tylenal \#3, and phenytoin were unsuccessful. Carbamazepine and phenytoin reduced the frequency and duration of painful crises to 3-4 times annually.

We developed a treatment plan consisting of a low dose morphine infusion with increasing dosage until pain was relieved.

Over the subsequent 28 months, we have had experience treating 7 crises with morphine given as $0.06 \mathrm{mg} / \mathrm{kg}$ IV push, followed by a continuous infusion of $0.02 \mathrm{mg} / \mathrm{kg} / \mathrm{hr}$ with amitriptyline $0.25 \mathrm{mg} / \mathrm{kg}$ hs. Pain control is immediate, with the infusion gradually tapered after 24 hours.

Despite good prophylactic agents, some patients with Fabry's disease continue to experience painful crises. Prompt treatment with adequate doses of intravenous narcotic is a fully effective acute treatment, and should not be avoided for fear of addiction or presumed partial efficacy.

P19.

Occurrence of Seizures as the Sole Manifestation of Organophosphate Toxicity

\section{E.C. WIRrell and K.E. Gordon (Halifax, Nova Scotia)}

Organophosphate insecticides are widely used in lawn sprays and household pest control. Toxicity from these agents is due to inhibition of acetylcholinesterase enzymes resulting in excessive stimulation of muscarinic, nicotinic and central receptors. Seizures as a result of stimulation of central receptors by acetylcholine are well described. They are generally assumed to be indicative of significant exposure and are accompanied by other signs of toxicity.

We report two children who were exposed to apparently "trivial" amounts of Chlorpyrifos, an organophosphate insecticide, and who developed seizures as their sole manifestation. In on case, repeated exposure to lawn sprays with subsequent seizures was documented on three occasions.

We conclude that in certain children, seizures in the absence of other symptoms or signs, may occur following mild organophosphate exposure.

\section{I-3. GENERAL NEUROLOGY}

P20.

\section{Definitions and Parkinson's Disease in the Young}

\section{A. JoSEPH and P.J. SWEENEY (Cleveland, Ohio)}

While Parkinson's Disease is essentially a disease of the elderly its occurrence in the earlier decades of life has long been known. Differing terminology used in the literature for the young onset population has resulted in considerable confusion. Dystonia, tremor, rigidity and bradykinesia before the age of 40 , with an immediate response to levodopa, are features of three previously described conditions: Segawa's Syndrome, Juvenile Parkinson's Disease (JPD) and Young Onset Parkinson's Disease (YOPD). Segawa's Syndrome with its onset in the first decade of life has dystonia as a prominent early feature. JPD, as used in the literature, encompasses all cases of idiopathic Parkinsonism with onset before age of 40 . YOPD, believed to be a subgroup of JPD has its onset of symptoms between 21 and 39 and also has dystonia as an early feature. YOPD is also characterized by earlier appearance of levodopa-related dyskinesias.

Parkinsonism in the young may consist of several disease entities with overlap of clinical features and diagnostic lables. Parkinsonism with its onset between the ages of 15 and 20 is a poorly understood entity. The historical evolution of the concepts of Segawa's Syndrome, JPD and YOPD will be outlined. 
P21.

\section{Ras Mediated Signal Transduction Pathway in Human Astrocytoma Cell Lines}

A. Guha, N. Lau and A. Pawson (Toronto, Ontario)

Cytosolic proteins (SHc, GRB2, sos) involved in activation of ras upon Platelet-Derived (PDGF) and Epidermal Growth Factor (EGF) stimulation have recently been cloned. The levels of these proteins, their activation (phosphoryllation) state and their interactions with PDGF/EGF receptors in astrocytomas is not known. Lysates from four malignant human astrocytoma cell lines (U-373, 343, 87, 138) were examined with immunoprecipitations and western blotting.

All cell lines expressed Shc, which was phosphoryllated with PDGF or EGF stimulation. Three cell lines expressed PDGF receptors, while all expressed EGF receptors. The receptors phosphoryllated with PDGF/EGF stimulation. Stimulated receptors complexed and phosphoryllated $\mathrm{SHc}$, which inturn bound GRB2. These results demonstrated the capability of activating the ras pathway in astrocytomas. Presently we are examining sos (a nucleotide exchange factor complexed with GRB2 that activates ras-GPD to ras-GTP), in these lines. Experiments to block ras using a dominant-negative mutant (asn 17), are also underway. If our results show that activation of ras is vital to the mitogenic signal in astrocytomas, reported pharmacological methods of blocking ras may be useful in decreasing the growth of astrocytomas.

\section{P22.}

Effect of Unexpected Changes in Target Location on Trajectories of Pointing Movements in Cerebellar Patients

R.G. Lee, B. Bonnefoi-Kyriacov, E. Legallet and E. Trouche (Calgary, Alberta; Marseille, France)

With cerebellar dysfuction the timing of events during a complex movement seems to be disturbed more than the mechanisms occurring during the planning period prior to the movement. To determine the role of the cerebellum in these different components of movement we examined 3 patients with cerebellar incoordination and 3 controls during a task in which they were required to point rapidly at a luminous target as soon as it appeared on a screen in front of them. In $25 \%$ of the trials the location of the target shifted suddenly during the preparatory period requiring the subject to make an adjustment in movement trajectory. Reaction time (RT), movement time (MT), and terminal accuracy were recorded. Movement kinematics were analysed using ELITE system. In the cerebellar patients RTs and MTs were prolonged and movement trajectories were irregular and variable. However, like the control subjects, the patients were able to change movement trajectory when the target shifted position, although the trajectory change was often delayed and inappropriately large. Thus cerebellar patients do seem capable of adapting motor programs after the initial preparation for movement has started, but the delays in these adaptations would be consistent with the notion that the cerebellum plays a role in the timing of voluntary movements.
P23.

\section{Repetitive Phrenic Nerve Stimulation for the Treatment of Persistent Hiccups}

\section{F. GRAND'MAISON and V. Soland (Sherbrooke, Quebec)}

Many different treatments have been proposed to alleviate persistent hiccups - from pharmacological therapy to various manoeuvres such as digital rectal massage, auriculo-acupunture and endoscopic massage of the cardia. Most of the suggested treatment modalities are either ineffective, slow-acting or induce significant side effects. In anecdotal reports, phrenic nerve stimulation has rapidly and safely suppressed persistent hiccups. We attempted this technique in a 35-year-old man with a left temporal astrocytoma who developed hiccups a few hours following a single dose of dexamethasone.

The phrenic nerve was stimulated transcutaneously in the neck 24 hours after the onset of hiccups. The diaphragmatic response was monitored with surface electrodes over the chest wall. Single supramaximal stimuli $(0.1 \mathrm{~ms}$ duration; $40 \mathrm{~mA})$ had no effect on the singultus. Repetitive stimulation of the nerve at $3 \mathrm{~Hz}$ and at $5 \mathrm{~Hz}$ was also ineffective. However, a single train of 10 stimuli at $10 \mathrm{~Hz}$ immediately abolished the hiccups. The patient has remained hiccup-free after a 3 month follow-up.

Repetitive stimulation of the phrenic nerve is a safe, rapid and effective option for the treatment of persistent hiccups in some patients. High frequency stimulation may be required.

\section{P24.}

\section{An EEG Classification for Coma}

G.B. Young, R.S. McLachlan and J.D. Demelo (London, Ontario)

A reliable EEG classification system is needed for the various electrographic patterns in comatose patients. Such a system should capture all the categories, recognize important differences and show a high degree of interobserver reliability.

One hundred EEGs from comatose patients were read in a blinded manner by 2 EEGers. One reading used the Synek system (J Clin Neurophsysiol 1988; 5: 161-174) with 16 categories. The other evaluation used our new classification system with 9 categories.

All EEGs were included with both classification systems; i.e., it was possible to categorize each recording. With the Synek system there was only $53 \%$ agreement between observers, $46.75 \%$ beyond chance alone (Kappa $=0.50$ ). With our system there was $83 \%$ agreement, $71.9 \%$ beyond chance alone (Kappa $=0.81$ ).

We have thus developed an EEG classification system that effectively captured all the cases and showed a significantly higher inter-observer reliability than a previously published study. 
P25.

\section{Acute Metabolic Stress In Vivo Modulates Cytokine Production from Glial Cells}

\section{L.D. KoJIC and J.E. CunNiCK (Ames, Iowa)}

There is an increasing range of clinical conditions in which cytokines, within the brain, are involved as part of disease pathogenesis, either as autocrine/paracrine growth factors or due to their role in disease progression. The possibility that stress plays an important role in the pathophysiology of the brain tissue damage was examined in a rat model. Exposure of adult, Lewis rats (12 wks old) to acute metabolic stress of 2-deoxy-D-glucose $(500 \mathrm{mg} / \mathrm{kg}$ ), resulted in physiological (elevated corticosteroids) and behavioral changes (prostration) indicative of stress. Saline injected rats served as controls. Primary cultures of unstimulated, mixed glial cells were assessed for phenotypic changes and changes in cell numbers. Parallel cultures were stimulated with LPS or media and assessed for proliferation, and ability to produce nitric oxide (NO) and tumor necrosis factor (TNF). Stress induced an increase (145-157\% of control) in numbers of unstimulated mixed glial cultures on day 5 and 8 (p's $<0.001$ ). Stress stimulated LPS-induced proliferation in mixed glial cells (p's < 0.0001 ) compared with the control group. Additionally, the production of TNF and NO were elevated in these cultures due to stress ( $p$ 's < 0.03). These data indicate that metabolic stress in vivo can modulate glial cell proliferation and responsiveness to LPS. Understanding the mechanisms that control activity of glial populations, especially at time of stress, may lead to new strategies to limit the extent and nature fo CNS tissue damage. Additionally, the use of normal adult brain cells and in vivo stress administration provides a new model in the study of CNS cytokine activity.

\section{P26.}

\section{A Left Hemisensory Phenomenon More Common in Women}

\section{T.G. StaunTon (Oakville, Ontario)}

Persisting sensory disturbance affecting one side of the face and the ipsilateral arm and leg usually leads to a series of often invasive investigations because of the possible etiologies, which might include tumour, vascular lesion, or demyelination. I present a series of 45 patients ( 38 women and 7 men) average age 42 yrs, seen and investigated over the last 8 years, whose presentations in terms of symptomatology, normal neurologic exam and investigations are strikingly similar. All complain of an abnormal, occasionally paresthetic sensation affecting the left side of the body, predominantly the face, neck and arm, more than the leg. The mean duration of symptoms prior to assessment was 3 years (ranging from 3 months to 15 years). None had any visual, motor coordination deficits. The sensation is described as a different, occasionally irritating, restless discomfort which is persistent but fluctuating in intensity, affected often by stress or anxiety. No underlying organic abnormality has been demonstrated despite intensive investigation. This condition appears to be a benign stress-related left hemisensory phenomenon, more common in women.
P27.

\section{Time Course of Changes in Axonal Function After Graded Experimental Spinal Cord Injury}

\section{H. Imamura, M.F. Fehlings and C.H. Tator (Toronto, Ontario)}

A clear appreciation of the time course of changes in axonal conduction and their relation to clinical neurological function after spinal cord injury (SCI) is of considerable importance in understanding the evolution of secondary injury events and also in appreciating the extent of functional plasticity within the traumatized spinal cord. Therefore, we investigated the clinical and electrophysiological recovery after graded clip compression at C8-TI in rats $[20 \mathrm{~g}(\mathrm{n}=8), 35 \mathrm{~g}(\mathrm{n}=7), 50 \mathrm{~g}(\mathrm{n}=7)]$. We recorded somatosensory evoked potentials (SSEP) and motor evoked potentials (MEP) before injury and every week after injury for up to 8 weeks. Simultaneously we assessed clinical motor function using both the Tarlov and inclined plane methods. An anatomical analysis of the injury site was performed by light and electron microscopy and by axonal tracing with fluorogold.

All injury groups showed considerable recovery of SSEPs and MEPs with time. This recovery correlated significantly $(\mathrm{p}<$ 0.05 ) with both open field walking as assessed by the Tarlov method and the inclined plane test. For example at one week after injury the latency of the SSEP was $108.7 \%(p<0.05)$, $125.2 \%(\mathrm{p}<0.01), 139.3 \%(\mathrm{p}<0.001)$ of control in the $20 \mathrm{~g}$, $35 \mathrm{~g}$ and $50 \mathrm{~g}$ groups respectively. By 8 weeks, the latency had recovered to $100.5 \%(\mathrm{p}>0.05), 105.0 \%(\mathrm{p}=<0.05), 108.8 \%(\mathrm{p}$ $<0.01$ ) of control. The results clarify the degree of functional plasticity after spinal cord injury and allow a clearer design and interpretation of neuroprotection studies.

\section{P28.}

\section{Development of an In Vitro Model of Spinal Cord Injury to Study Neuroprotection}

\section{M.G. FeHLINGS and R. NASHMI (Toronto, Ontario)}

To examine fundamental issues relevant to the study of neuroprotection in acute spinal cord injury (SCl), we have developed an in vitro model of SCI in isolated adult mammalian spinal cord axons. A $20 \mathrm{~mm}$ segment of thoracic spinal cord is exposed by laminectomy and rapidly removed into cold $\left(5^{\circ} \mathrm{C}\right)$ oxygenated $\left(95 \% \mathrm{O}_{2} / 5 \% \mathrm{CO}_{2}\right)$ Ringer's solution. The dorsal columns are then isolated with iris scissors and placed in a temperature controlled $\left(25^{\circ} \mathrm{C}\right)$ recording chamber. Field potentials are recorded at two points using glass microlectrodes (IM $\mathrm{KCl}$; 1-2M $\Omega$ ) following stimulation with a bipolar Pt electrode (1-3 $\mathrm{mA} ; 0.2 \mathrm{~Hz} ; 100 \mu \mathrm{s})$. The dorsal column segment was injured $(\mathrm{n}=10)$ for $15 \mathrm{sec}$ using a modified aneurysm clip calibrated with a closing force of $2.0 \mathrm{~g}$. Following injury we observed: a $43 \%$ decrease in amplituded ( $<<0.001)$; a $131 \%$ increase in response latency $(\mathrm{p}<0.001)$; and significant alterations $(\mathrm{p}<$ $0.05)$ in the refractory period and ability to conduct high frequency $(>200 \mathrm{~Hz}$ ) trains. Furthermore, the axons show physiological (enhanced response to the $\mathrm{K}^{+}$channel blocker 4-aminopyridine) and ultrastructural evidence of myelin sheath disruption. We conclude that this model of in vitro injury to isolated adult mammalian spinal cord axons provides an excellent 
method to study neuroprotection strategies at a very fundamental level.

Supported by the Medical Research Council of Canada.

P29.

\section{Visual Evoked Potentials Versus Perimetry in Assessment of Chiasmal Compression from Pituitary Adenoma}

M.S. Jog, B.D. Archer, N. Shampur and J.E. Turley (Hamilton; Toronto, Ontario)

Functional assessment of the visual pathway from compressive pituitary lesions is limited to visual field perimetry. Accuracy of measurement and interpretation of severity of compression is difficult. Confounding factors include reproducibility of the examination, sensitivity and availability of the equipment, technician expertise, patient cooperation, and ease of obtaining and interpreting data. Visual evoked potentials (VEPs) are virtually free of all of the above difficulties, are reproducible and non invasive. This study compares visual perimetry with full field and nasal and temporal half field VEPs in the detection of chiasmatic compression.

15 patients without visual symptoms underwent coronal computerized tomography, visual perimetry and full and half field VEPs. 9 patients had microadenomas and had normal VEPs and perimetry. 6 patients had macroadenomas. All VEP recordings were abnormal in two of these patients with 1 having abnormal perimetry. The remaining 4 patients had normal perimetry, full and nasal VEPs but abnormal temporal hemifields. These findings demonstrate superiority of VEPs over perimetry in assessing asymptomatic chiasmal compression from pituitary macroadenomas $(p=.03)$. Half field temporal VEPs may be better at detecting these subtle changes than full field VEPs $(p=.06)$. Test-rest reliability of all VEP studies was 0.89 which makes the test highly reproducible.

P30.

The Association of Buspirone and Carbamazepine is Effective in Early Post-Traumatic Delirium

E. Pourcher, M.J. Filteau, R.H. Bouchard, P. Baruch and J.P. BOUCHARD (Quebec, Quebec)

Brain injury with severe brainstem contusion and long-lasting loss of consciousness may cause complex neurobehavioral disturbances in the early post-acute period. Agitation and disorientation are major problems interfering with nursing and early rehabilitation programs. Major tranquilizers such as haloperidol prescribed to treat aggressive outbursts and erratic driveness may increase akathetic agitation and confusion, or induce parkinsonism.

We report four cases of agitation and aggressiveness in young patients (aged from 19 to 26 years) with acute braininjury in whom both behavioral disruption and confusion were rapidly improved by the association of buspirone $10 \mathrm{mg}$ three times/day and carbamazepine CR $200 \mathrm{mg}$ twice/day. A quieting effect was obtained in 12 to 36 hours, with a better cooperation to nursing. No more hallucinatory behavior was observed.
Attention was evaluated with serial sevens and short-term memory was assessed by serial digit spans: both were improved after 48 hours of this regimen.

This is the first report of the potential synergism of buspirone and carbamazepine in improving agitation, attention and shortterm memory in post-acute close-head injury delirium. both drugs modulate 5HT transmission any may affect limbic neurotransmission functionally altered by early and late traumatic events.

P31.

\section{Rare Clinical Presentations of Neurosarcoidosis}

J. Ravindran, C.A.J. Boyle, J.M. Radhi, D. George, K.H. NAIK and A.H. RAJPUT (Saskatoon, Saskatchewan)

Neurologic manifestations occur in $5 \%$ of cases of sarcoidosis which can be the presenting manifestation or the only manifestation of sarcoidosis. We report 2 cases of neurosarcoidosis (NS) with rare presenting manifestations. Case 1: A 31-year-old male presented with dementia of 4 month duration and no focal neurologic signs. MRI showed diffuse meningeal enhancement with no parenchymal lesion. Investigations revealed bilateral hilar adenopathy (positive for granulomas), diabetes insipidus and elevated CSF protein and lymphocytic pleocytosis. Case 2: A 65-year-old lady presented with leg weakness, sacral anaeshesia and sphincter incontinence. MRI showed a contrast enhancing lesion in the conus medullaris. Biopsy of the lesion revealed sarcoidosis. Initial presentation with dementia and no parenchymal MRI lesion or focal neurologic sign has not been reported. NS should be considered in the differential diagnosis of dementia and subacute or chronic myelopathy. MRI and CSF findings are not specific. Diagnosis is best made by aggressive search for extraneurologic manifestations of sarcoidosis and characteristic histopathology of non caseating granulomas.

\section{P32.}

Apnea Complicating Arnold Chiari Malformation: Report of Two Cases

S. Omer, M.Z. Alkawi, A. Bouchama, S. Bohlega and D. MCLEAN (Riyadh, Saudi Arabia)

The Arnold Chiari malformation (ACM) is associated with two types of apnea in children; an obstructive apnea due to bilateral abductor paresis of the vocal cord, and a central apnea. In recent years, reports of an association between ACM and both central and obstructive sleep apneas in adults have been forthcoming. We report the cases of two adult patients who developed hypercapnic respiratory failure followed by respiratory arrest. Both patients required mechanical ventilation. Both patients had physical signs suggestive of a pathology in the craniocervical junction. The diagnosis of ACM was confirmed in both of them by CT myelography and/or MRI.

Appropriate imaging investigation of the craniocervical junction should be part of the workup of patients with unexplained apneic episodes. 
P33.

\section{Abnormal SPECT Scans and Impaired Memory in Patients with Adjuvant Breast Disease}

S. Brown, B.M. Patten, N. Cooke, B. Ostermeyer Shoaib and S.G. JHINGRAN (Houston, Texas)

Among the first 100 patients with silicone breast implants that we evaluated 81 patients reported memory problems. We therefore started to investigate patients with breast implants and memory problems with SPECT scans and psychological testing. We report here the results of 15 implanted women with systemic autoimmune disease who received a SPECT scan and psychological testing. SPECT scans were digitalized at the midcerebellar transaxial plane and that region compared to 8 regions of the cortex at the midventricular transaxial slice. All patients underwent California Verbal Learning Test, Wechsler Adult Intelligence Scale-Revised, Timed Sustained Attention Test and Verbal Fluency Test at the same time. Occipital regions were normal (Ratio $\mathrm{R}=0.962, \mathrm{~L}=0.937$ ), but other regions including bilateral temporal showed decreases which were statistically significant using paired t tests. Repeated scans after removal of the implants showed significant improvement in most patients. On the California Verbal Learning Test, performance was below average for verbal learning, short and long delayed free recall and delayed recognition. In contrast, performance on tasks requiring attention, language and intelligence was average. Women with implants can develop impaired memory and abnormal SPECT scans. Further studies are warranted.

\section{P34.}

\section{Chronic Optic Neuropathy from Intracranial Contrast Media}

W.A. Fletcher, S.K. MukherjeE and R.J. Sevick (Calgary, Alberta)

Intracranial radiographic contrast media is an unrecognized cause of delayed optic neuropathy. We report a case of bilateral chronic optic neuropathy presenting 30 years after subarachnoid spread of contrast medium.

In 1991, a 44-year-old woman presented with blurred vision in her right eye for 8 months. In 1960, at age 14, she had a left frontal brain abscess, which was treated with 3 needle aspirations followed by injection of radio-opaque contrast medium. Post-injection $\mathrm{x}$-rays showed contrast medium along the falx and outlining one optic nerve. The abscess was resected 3 weeks later. Visual acuity in 1991 was 20/25 OD and 20/15 OS with a right relative afferent pupillary defect $(1.2 \mathrm{log}$ units). Perimetry showed an absolute inferior altitudinal hemianopia OD and a dense inferior nasal wedge-shaped defect with a nasal step OS. There was moderate right and mild left optic atrophy. CT scan showed a rim of high density encircling both optic nerves from orbital apex to globe and an irregular layer of high density on the tentorium. In January, 1994, visual acuity and fields and optic atrophy were unchanged but the RAPD had increased to $2.5 \log$ units.

Myelography with iodized oil has rarely caused an acute or subacute optic neuropathy. Chronic neuropathy has been reported only once before (Occhiogrosso et al. 1979) and led to bilateral blindness 3 years after Pantopaque myelography.
P35.

Adult Bilirubin Encephalopathy (Kernicterus): A Report of Two Cases and a Review of the Clinical Course, Treatment and MRI Findings

C. Yegappan and T. Curran (St. John's, Newfoundland; Vernon, British Columbia)

Adult bilirubin encephalopathy (BE) is rarely seen and/or recognized in modern day neurology practice. Involuntary movements, gaze abnormalities and sensorineural hearing loss are the characteristic features of BE. Haemolytic disease ( $\mathrm{Rh}$ incompatibility) producing severe bilirubinemia is now an uncommon cause and has been replaced by the controversial mild-moderate hyperbilirubinemia in term and preterm infants as the leading cause of $\mathrm{BE}$.

We report two cases of $\mathrm{BE}$ : the first is a 41 -year-old male born with $\mathrm{Rh}$ incompatibility induced hyperbilirubinemia who has athetosis and a supranuclear gaze palsy. The second is a 21-year-old female who was born three months premature and had protracted jaundice. She has significant dyskinesias, a supranuclear gaze palsy and bilateral hearing loss.

We will review the current literature pertaining to BE and report on the clinical adult course and the treatment modalities available including botulinum toxin. Additionally we will present the results of our MRI studies which to our knowledge have not previously been reported.

P36.

A Randomized, Placebo Controlled, Double Blind CrossOver Trial of Amantadine in Autosomal Dominant Cerebellar Ataxia

R.K. Mosewich, J.R. Donat and R. Bourgault (Saskatoon, Saskatchewan)

Autosomal Dominant Cerebellar Ataxia (ADCA) is a heterogeneous degenerative disorder. Work has recently lead to progress in characterizing the molecular pathogenesis of this group of diseases. Unfortunately, no therapy has been proven beneficial in limiting the symptoms or the progression of ADCA. Some reports have suggested Amantadine is symptomatically useful though others have not.

We performed a randomized, placebo controlled, double blind cross-over study in 6 patients with ADCA who belonged to the same family. Patients were first tested at baseline with a finger tapping test, the Grove Peg Board Test, a timed 50 foot walk, a video taped neurologic exam and a subjective symptom questionnaire. Patients then received either Amantadine at a dose of 100 milligrams twice daily or placebo for 2 weeks and were re-studied. After a 2 week washout period the same procedure was performed with each patient receiving the alternate medication.

There were no significant differences in any parameter after a treatment with Amantadine or placebo. Subjectively, 3 patients on Amantadine felt they improved mildly, 2 felt they worsened and one felt unchanged. Amantadine therefore appeared to be of no benefit in our ADCA patients. 


\section{SESSION II}

\section{II-1. EPILEPSY}

P37.

\section{Postictal Enhancement on MRI}

A.A. WILfong, N.J. LowRY and J.Y. YAGER (Saskatoon, Saskatchewan)

Transient neurologic deficits are well known following partial seizures. Clinically these include alterations of sensorium, somatosensory abnormalities and motor paralyses. Appropriately timed electroencephalography (EEG) documents the associated focal slowing and depression of background that correlates with the area of brain involved in the seizure discharge. This area can also be identified due to regional enhancement seen on postictal magnetic resonance imaging (MRI) of the brain following Gadolinium infusion.

An illustrative case is presented: T.H. is an eight-year-old girl who had a medulloblastoma resected followed by craniospinal $\mathrm{x}$-ray therapy and chemotherapy in 1990. She had an excellent result and was disease-free with a normal neurologic examination. In late-1993, T.H. fell and sustained a closed head injury without loss of consciousness. Over the ensuing seven days she had headaches, nausea and vomiting. On the eighth day she began to have recurrent complex partial seizures. EEG the following day revealed depression and slowing over the left hemisphere and CT scanning before and after contrast was normal. MRI with Gadolinium infusion three days later demonstrated marked gyriform enhancement of the left occipitotemporal region. Repeat MRI six weeks later was normal.

Localization of a partial seizure discharge is possible using postictal MRI with Gadolinium enhancement.

\section{P38.}

\section{Control of Intractable Photic Epilepsy with Eye Patching}

\section{A.A. WILFONG (Saskatoon, Saskatchewan)}

Although photic sensitive epilepsy is rare, it is the commonest type of reflex epilepsy. Most patients attain control of their seizures with antiepileptic medications such as valproic acid, ethosuxamide and/or clobazam. However, a significant number prove to have medically intractable seizures. In these patients, adjunctive measures aimed at altering the provoking visual stimulus may be helpful. Polarized sunglasses are the most popular method, but may not control seizures in severe cases. Monocular eye patching is an effective alternative.

An illustrative case is presented: R.M. is a seven-year-old boy who began to experience myoclonic seizures in response to photic stimuli shortly after birth. The myoclonic jerks were not recognized as epileptic until two years of age when he had his first generalized tonic-clonic seizure while watching television. Subsequent treatment with phenobarbital, valproic acid, ethosuxamide, and clobazam, and also wearing polarized suglasses did not stop the seizures. However, seizure control was attained with monocular eye patching. The electroencephalographic (EEG) photoparoxysmal response to flickering light was abolished while wearing an eye patch.
Monocular eye patching is an effective adjunct in the management of photic sensitive epilepsy. Abolition of the EEG photoparoxysmal response may identify those patients who will benefit most.

P39.

Telephone EEG: Comparison of the 2-Wire and 4-Wire Recordings

A. Ogunyemi, J. Goodwin and A. Hopkins (St. John's, Newfoundland)

Several questions remain unanswered regarding the technique of telephone EEG. There are concerns related to (i) unfavourable signal-to-noise ratio, (ii) deformation of EEG potentials, (iii) artifacts from telephone lines, (iv) interchannel cross-talk and (v) inadequate number of channels. In a recent investigation, we observed that EEG tracings recorded following transmission through 4-wire teleconference network were of acceptable quality for clinical use. In the present study, we compared the quality of EEG tracings obtained simultaneously through the 2-wire (public telephone lines) and the 4-wire (dedicated, teleconference) systems.

Data from eight-channel EEGs were transmitted via telephone lines over a distance of about 900 kilometres (from St. Anthony to St. John's, Newfoundland) for 48 consecutive patients. Identical recording characteristics (sensitivity, lowfrequency and high-frequency filters) were used to accomplish the recordings from the 2-wire and 4-wire systems.

EEG background activities were well visualized in all the tracings obtained via the 4-wire network. Only one tracing from the 2-wire system was unsatisfactory for visual analysis. Epileptiform and non-epileptiform abnormalities and artifacts, related and unrelated to telephone transmission were adequately identified. Baseline drift and interchannel cross-talk were rare. Signal-to-noise ratio was less favourable in the 2-wire recordings.

\section{P40.}

\section{Jacob Creutzfeld Disease Complicated by Refractory Status Epilepticus}

F. Souchon, L.-E. Roy, P. Laplante, J. Bolleau, J. Lachapelle, A. Robillard, S. Rousseau and J. Teitelbaum (Montreal, Quebec)

Objective: Discuss a case of Jacob Creutzfeld Disease complicated by early refractory status epilepticus and review related literature.

Background: JCD is a rare form of dementia transmitted by a proteinaceous infective particle (PRION). The disease classically presents with a rapidly evolving dementia, myoclonus and characteristic EEG findings. In a review of 230 cases, Brown and coll. found that two-thirds of these patients presented with an early deterioration of cognitive function, one-third with exclusive neurologic signs and only $0.4 \%$ with seizures as their first symptom. Only $8 \%$ of the group presented any form of seizures during the course of the illness.

Method: Review the medical record of a pathologically confirmed case and of pertinent literature. 
Results: A 58-year-old female patient developed a refractory status epilepticus after two weeks of a progressive neurological syndrome characterised by ataxia and abnormal behavior which suggested a psychotic illness and justified an early reference to a psychiatrist. After three weeks of unsuccessful medical management of her seizures, a brain biopsy showed typical JCD abnormalities. This association has not been described in the English literature.

Conclusions: Status epilepticus should include JCD as a rare etiology.

\section{P41.}

\section{Touth-Brushing Epilepsy}

\section{J. BRUNI (Toronto, Ontario)}

Reflex seizures are precipitated by clearly defined stimuli: most frequently reflex seizues are light stimulus induced (photosensitive seizures) and are generalized. This report reviews an unusual case of tooth-brushing epilepsy in an 52-year-old woman. The patient presented with left sided tinnitus and a tingling sensation of the left side of her body which occurred with brushing of her teeth. Initially there was no alteration of consciousness with these spells but subsequently complex partial seizures developed. The patient had a normal neurologic examination, CT scan and EEG. The patient was treated with carbamazepine with an initial good response. The patient's seizures subsequently became more refractory. Repeat neuro-imaging studies revealed a space-occupying lesion in the right parietal lobe. Four years after initial presentation the patient underwent partial excision of a tumour that had undergone malignant transformation (glioblastoma multiforme). This is the first case report of tooth-brushing epilepsy in association with a structural CNS lesion. One other patient (Epilepsia 23: 657-661, 1982) had developed tooth-brushing induced seizures several weeks after placement of a palatal expansion device which irritated the upper gums. The possible mechanisms of this unusual type of reflex epilepsy will be discussed.

\section{P42.}

Anti-Epileptic Drug (AED) Withdrawal During Video-EEG Monitoring in Candidates for Epilepsy Surgery. Elaboration of a Standardized Protocal

S.Tremblay, R. Desbiens, C. Robitaille and L. Nadeau (Quebec, Quebec)

Reduction of AEDs in order to record seizures during videoEEG monitoring is part of the standard pre-surgical evaluation in candidates for epilepsy surgery. It provides recording of several seizures within a few days, allowing clinical-EEG correlations and localization of seizure onset.

Two important concerns are efficiency and safety: recording time should be shortened because of discomfort, cost of hospitalization and limited availability of equipment. However, status epilepticus and post-ictal psychosis should be prevented.

Theoretically, AED discontinuation following a standardized protocol should be superior to empirical method: while being safe and flexible, it should shorten acquisition of meaningful information and diminish potential sources of errors among the medical team.

We devised such a protocol in which:

-AED are withdrawn in a pre-determined order and at a specific rate

-Through AED serum levels are measured daily

-Seizure types and severity are tabulated.

End points are positive (recording 5 or more meaningful seizures) and negative (status epilepticus, post-ictal psychosis, seven days of recording with less than 5 seizures).

We devised a standardized protocol which will be compared to empirical AED discontinuation in 100 patients assigned randomly to one method or the other. Our methodology and preliminary results will be presented.

\section{P43.}

Temporal Lobe Originating Partial Complex Seizures with "Frontal" Lobe Features

\section{R.M. SADLER AND W.J. Howes (Halifax, Nova Scotia)}

Features of complex partial seizures of frontal lobe origin (FLCPS) have been reported (Williamson et al., Ann Neurol $1985 ; 18: 497-504$ ) and include brief, frequent attacks, vigorous motor and sexual automatisms, vocalization, partial complex status epilepticus, and little postictal confusion. We report a patient with similar characteristics but of temporal lobe origin.

A 21-year-old male had a 15 year history of medically intractable epilepsy. Seizures began with a brief epigastric sensation followed by a loss of awareness and complex motor features (see below). Seizures occurred in clusters of 5-15 attacks per 24 hours (awake and sleep) every 2-3 months.

Scalp video-EEG telemetry seizures demonstrated a loud cry followed by marked bilateral violent kicking, genital manipulation, and reactive automatisms. Seizure duration was $40-60 \mathrm{sec}-$ onds. Postictal confusion was present. Interictal and ictal EEGs showed a left anterior temporal focus. A left inferior-medial temporal cavernous angioma was found on MRI scan. In November 1989 the patient had a resection limited to the anterior $3 \mathrm{~cm}$ of the left temporal lobe and the vascular lesion. He has been seizure free on carbamazapine except for rare auras and 4 seizures (of unknown type) in Jan-Feb 1993 due to medication noncompliance.

The "typical" manifestations of FLCPS may be characteristic but are not specific for frontal foci and can be seen with seizures of temporal origin.

\section{P44.}

Predictive Accuracy of Late Lateralizing EEG Features in Localizing Scalp Recorded Temporal Lobe Seizures

J. Ravindran, N. Lowry, M. Kaibara and W.T. Blume (Saskatoon, Saskatchewan; London, Ontario)

We studied the predictive accuracy of late lateralizing EEG features for localizing seizure focus in 39 consecutive patients who had temporal lobectomy and remained seizure free for 2 years. Seizures which were pre-operatively localized based on a clear focal onset were excluded. Late lateralizing features were considered to be evolution of rhythmic waves or repetitive 
epileptiform discharges. The side of successful temporal lobectomy was used as the gold standard for assessing the correct localization. There were 148 ictal recordings without a clear focal onset occurring in 39 seizure free temporal lobectomy patients. Ninety-one out of the 148 recordings showed late lateralizing features. Eighty seven out of the 91 (95.6\%) with late lateralizing features correctly predicted the seizure focus in temporal lobe (64) or hemisphere (23). Thirty-five out of 39 patients $(89.7 \%)$ had one or more seizures lateralized exclusively ipsilateral to temporal lobectomy suggesting correct prediciton. Thrity-nine out of 52 seizures $(75 \%)$ showed late lateralizing features within 20 seconds of onset. Although of limited value when considered alone, late lateralization of these seizures to one temporal lobe or hemisphere can add important information to the congruence of the data in pre-operative evaluation of temporal lobe epilepsy.

\section{P45.}

Complex Partial Seizures and Drop Attacks Provoked by Posterior Mesial Temporal Lesion: Coherence Analysis and Frameless Stereotactic Epilepsy Surgery with 3D CT and EEG Assistance

H. Otsubo, M. Steinlin, M. Cortez, A. Hunjan, P.A. Hwang, V. JAY, L.E. Becker, S. Holowka, S.H. Chuang and H.J. HOFFMAN (Toronto, Ontario)

Digital EEG analysis with prolonged video telemetry and epilepsy surgery supported by three dimensional (3D) CT with EEG electrode markers were performed for the five-year-old boy with refractory seizures. He presented with three year history of complex partial seizures of staring, and head drops with tonic flexion of the arms. Complex partial seizures were corresponded to the leading spikes from the right temporo-parietal regions with secondary generalized. During drop attacks, EEG showed paroxysmal generalized spike and waves. Coherence study analyzed secondary bilateral synchrony of right hemispheric leading. CT and gadolinium MRI demonstrated calcification and negative enhanced increased signal intensity in the right mesial temporal lobe. Contrast CT with EEG electrode markers in the epileptogenic zone for $3 \mathrm{D}$ imaging was performed. The surface electrocorticography and depth electrode toward the mesial temporal region were applied for the lesionectomy combined with resection of epileptogenic mesial and inferior temporal regions, using frameless stereotaxy. Pathology confirmed a low-grade mixed glioma and cortical dysgenesis. This paper discusses about the coherence analysis which proved ipsilateral side of the lesion leading, and resection of the lesion combined with epileptogenic zone using frameless stereotactic surgery guided by $3 \mathrm{D}$ CT with EEG electrode markers.

\section{II-2. CEREBROVASCULAR}

P46.

\section{The Stereotactic Approach to Intracrainial Aneurysms}

M. Munz, F.B. Meyer, T.M. Sundt and P.J. Kelly (Montreal, Quebec; Rochester, Minnesota; New York, New York)

Although the pterional approach to anterior circulation aneurysms had been described in 1937 by Dandy, certain aneurysms may need to be approached differently either because of their location or due to associated lesions. We describe a series of five patients who had six intracrainial aneurysms treated through a stereotactic approach using the Compass Stereotactic system. The patients were treated between November 1990 and September 1992 at the Mayo Clinic. There were four females and one male. Four patients presented with subarachnoid hemorrhage and one with a seizure disorder. In two patients the aneurysm was associated with an arterial venous malformation. In both of these patients the subarachnoid hemorrhage upon presentation was thought to be due to rupture of the associated aneurysm. One patient presented with two mycotic aneurysms secondary to congenital heart disease. In the four patients who presented with subarachnoid hemorrhage there was an associated intraparenchymalor intraventricular hematoma. Patients ranged in age from 18 to 54 years with a mean age of 41 . Follow up was from 2 to 16 months (ave. 8 months). The location of the aneurysms had the following distribution: three were located on the distal middle cerebral artery, one each on the superior cerebellar artery, the posterior cerebral artery and the posterior lateral choroidal artery. All patients were imaged post operatively with both computed tomography and cerebral angiography to confirm obliteration of the aneurysm. Four of the patients are significantly improved following surgery, one patient who was severely affected preoperatively remains in stable condition. In selected patients with intracrainial aneurysms in whom the aneurysm may be difficult to localize a stereotactic approach may be helpful in their localization and treatment of both the aneurysm and associated pathology such as arterial venous malformations or intracerebral hematoma.

\section{P47.}

\section{Brain Stem Cavernous Malformations: A Skull Base Management Strategy}

M.G. Hamilton, G. Thompson, J. Golfinos, J. Zabramski and R. SPETzler (Calgary, Alberta; Phoenix, Arizona)

Cavernous malformations are vascular abnormalities that affect less than $0.5 \%$ of the population. Although they can involve any part of the central nervous system, only infrequently is the brain stem involved. Furthermore, because of the intricate anatomy of the brain stem, and the complexities associated with obtaining surgical access, these lesions have only recently been considered surgically treatable. The initial consideration for each individual patient involved the determination of whether surgery is indicated, followed by the evaluation of whether surgical removal is technically feasable with reasonable treatment risk. An essential component of surgical planning is to select lesions that point at the surface of the brain stem and approach these lesions in a direct fashion, along a line that is perpendicular to the bulk of the malformation and intersecting at the site of pointing on the brainstem surface. Utilizing this standardized management protocol and skull base surgical strategy, 35 patients have undergone resection of a cavernous malformation involving the brain stem: midbrain, $\mathrm{n}=9$; ponto-mesencephalon, $n=3$; pons, $n=6$; pontomedullary junction, $n=4$; and the medulla, $n=4$. The treatment results have on the whole been satisfactory: 27 patients were improved or neurologically 
stabilized, 6 patients were neurologically worse and 2 patients died. We feel that utilization of this management strategy, and skull base techniques where needed can allow the successful management of many patients with cavernous malformations involving the brain stem.

P48.

\section{Pediatric Cerebral Arteriovenous Malformations: A Management Outcome Comparison With an Adult Cohort}

M.G. Hamilton, D. Karahalios, G. Thompson, H. Rekate and R. SPETZLER (Calgary, Alberta; Phoenix, Arizona)

Management decisions for patients with cerebral arteriovenous malformations (AVMS) are typically based upon an evaluation of the complexity of the AVM, as identified by the Spetzler and Martin grade, and patient factors such as the general physical, and neurological condition. The Spetzler and Martin grading system was prospectively applied to a group of 121 consecutive patients undergoing complete surgical obliteration of their AVM. Patients were assessed with post-treatment angiography. Twenty-three pediatric patients were treated, 14 boys and 9 girls, mean age 11.5 years. There was no significant difference between the pediatric group and 98 adult patients with regard to sex distribution, clinical presentation, AVM size, or AVM grade. There was a significantly higher number of AVMs involving the basal ganglia and thalamus in the pediatric group $(\mathrm{p}<0.007)$. The number of treatment stages for the pediatric patients increased from a mean of 1.0 for grade I AVMs to 3.3 for grade $\mathrm{V}$ AVMs with no significant difference demonstrated when compared with the adult group. Mean followup was 1.0 year. Treatment outcome was significantly better in the pediatric group compared with the adult group: no significant morbidity occurred in the pediatric patient, while an AVM grade dependent morbidity was demonstrated in the adult group (3.5\% in grade III patients and $27.3 \%$ in grade IV and V patients; $\mathrm{p}=$ $0.04)$. Pediatric patients, therefore, had a significantly better outcome for treatment of compared AVMs, when comapred to adult patients. A management strategy will be presented.

\section{P49.}

\section{Management of Deep AVMs Involving the Thalamus, Basal} Ganglia, and Brain Stem

M.G. Hamilton, M. Lawton and R. Spetzler (Calgary, Alberta; Phoenix, Arizona)

Arteriovenous malformations (AVMs) located in the basal ganglia, thalamus, and brain stem have a propensity to cause devastating hemorrhage, and present a complex management problem for neurosurgeons. Thirty-two patients with deep AVMs were treated: 7 with AVMs in the basal ganglia, 11 in the thalamus, 4 in both the basal ganglia and the thalamus, 8 in the brain stem, and 2 in the brain stem and thalamus. The majority of the malformations were high (Spetzler and Martin) grade AVMs: grade I, $\mathrm{n}=0$; grade II, $\mathrm{n}=2$; grade III, $\mathrm{n}=7$; grade IV, $\mathrm{n}=10$; and grade $\mathrm{V}, \mathrm{n}=13$. Hemorrhage was the most common clinical presentation (84\%), and neurological deficits were common $(69 \%)$. Surgical treatment consisted of either direct resection (single stage, $\mathrm{n}=7$; multiple stages, $\mathrm{n}=11$ ), or indirect obliteration using intraoperative embolization $(n=11)$. Transfemoral embolization was performed in 18 patients, and stereotactic radiation was utilized in 10 patients. Complete AVM obliteration was accomplished in 19 patients but was incomplete in 13 patients. There was no operative mortality and treatmentassociated neurological deterioration occurred in 5 patients (16\%). The ultimate goal of treatment is complete removal of the AVM which is best achieved by direct surgical resection, utilizing staged procedures and multiple surgical approaches as needed. However, treatment risks must be carefully balanced against natural history. A amanagement strategy for these complex lesions will be presented.

\section{P50.}

Endovascular Therapy for Patients with Cerebral Arteriovenous Malformations and Progressive Neurologic Deficit

M.G. Hamilton, P. Apostolides, M. Khyata, R. Spetzler and A. WAKHLOO (Calgary, Alberta; Phoenix, Arizona)

The gold standard for treatment of cerebral AVMs is complete excision or obliteration. The risks associated with the treatment of patients with high grade AVMs can approach $20 \%$, which may exceed the natural history in patients who have not yet experienced hemorrhage from the AVM. We have used endovascular procedures to treat six such patients who presented with progressive neurological deficit. The aim of this approach was to control symptoms without subjecting the patient to the significant risks associated with a more aggressive management strategy. Four male and two female patients, mean age 30 years, had four (Spetzler and Martin) grade V AVMs, and one each of grade III and IV AVMs. All six patients presented with progressive extremity numbness and weakness associated with progressive facial weakness $(n=4)$, speech difficulty $(n=2)$, visual change $(n=1)$, slowed cognition $(n=2)$, or worsening seizures $(n=2)$. Patients were treated with either $n$-butyl cyanoacrylate or polyvinyl alcohol utilizing a transfemoral approach. The mean followup has been 5.5 months (range 2 to 12 months). Five patients developed transient worsening of their neurological deficit for up to one week post-embolization. At one month, five patients had marked improvement in their motor strength, and one patient remained unchanged. There were no deaths or hemorrhages. Endovascular therapy is an effective option for patients who have high grade cerebral AVMs and progressive neurologic deficit.

\section{P51.}

\section{Cerebral and Subarachnoid Haemorrhage in Pregnancy}

\section{R. LEBLANC (Montreal, Quebec)}

With obstetrical advances purely intracranial causes of cerebral and subarachnoid haemorrhages (SAH) have increased in relative frequency in the pregnant patient. The magnitude of this occurrence is illustrated by 6 cases relecting the spectrum of intracranial pathology that can cause these haemorrhages in the gravid woman. The patients were aged 24-35 years (mean 29.8). 
Two were in the 1st trimester and 4 were in the 3 rd trimester of a 1st, 2nd, 3rd, 4th, 5th and 7th pregnancy. One patient had a SAH of unknown etiology, the others had an intraparenchymal haemorrhage from an AVM (3 cases), from an aneurysm (1), and from recurrent haemorrhage in an oligodendroglioma (1). Four patients were Grade I at admission, 1 was Grade III and I was Grade IV. The aneurysm patient and the tumour patient, both in their 3rd trimester, had an emergency craniotomy and cesarian section, 1 AVM patient had an emergency craniotomy and elective cesarian section at term, 1 had and elective cesarian section and another a spontaneous delivery at term followed by subsequent treatment of an AVM, and the patient with a SAH subarachnoid haemorrhage of unknown etiology was treated by bed rest and elective cesarian section at term. All babies were normal at birth and none of the mothers had a neurological practice can frequently produce a satisfactory outcome in the gravida.

P52.

\section{Endovascular Treatment of Basilar Aneurysms With Detachable Coils}

J. Raymond, D. Roy, S. Fontaine, G. L'espérance, M. BoJANOWKSI, L.H. LEBRUN and J. TROPPER (Montreal, Quebec)

Background: Basilar aneurysms are difficult lesions to clip; they are often easy to approach by the endovascular route and can be treated with Guglielmi Detachable Coils (GDC). If this new approach is to be of some clinical value, it should be first noticed in the management of aneurysms at this site.

Method: Prospective analysis of angiographic results and clinical outcome at 6 months in patients referred for endovascular treatment of basilar aneurysms.

Patients: There were 13 patients, 6 males and 7 females. Eleven patients had bled and 10 patients were treated during the acute phase. The Hunt and Hess clinical grades were: Grade II: 2 patiens; Grade III: 7 patients; Grade V: 1 patient.

Results: The patient Grade $\mathrm{V}$ at presentation died on day 7 despite complete obliteration of the lesion without complication. There was one significant complication, perforation of the aneurysm, with clinical aggravation and death at day 9 .

The aneurysm was completely obliterated with no significant complication in 11 patients. The 6 months follow up angiogram available in 10 patients showed only one minimal aneurysmal neck recurrence and stable morphological results in 9 patients. The Glasgow outcome scale of these 13 patients was: Grade I: 11 patients, Grade V: 2 patients.

Conclusion: The procedure was successful in $92 \%$ of cases with one GDC related complication (8\%). Clinical and angiographic results were excellent at 6 months follow up in $85 \%$. This early clinical experience suggests that if morphological remains stable on long term follow up ( $>1$ year), the endovascular treatment may become the favored approach in the management of basilar aneurysms.
P53.

\section{Association Between Giant and Multiple Aneurysms}

\section{K.L. Chandler and M.W. Ray (Memphis, Tennessee)}

A retrospective analysis of aneurysm patients treated over a 14 year period was done on 257 records. In 19\%, patients had multiple lesions while in $6 \%$, solitary giant aneurysms were present. Another $5 \%$ had both a giant $(>2.5 \mathrm{~cm})$ and at least one other aneurysm associated with it. In this group, only two patients presented with typical signs and symptoms of subarachnoid hemorrhage secondary to rupture of the smaller lesion. None of the giant aneurysms acutely bled although hemosiderin staining and adhesions were noted at the time of surgery. A myriad of symptoms referable to the giant aneurysm were noted including TIA-like symptoms, visual acuity problems and seizures. In most cases, the location of the smaller was not related to the giant aneurysm. No other associated systemic illnesses were found. All lesions were surgically treated via clipping, CUSA of carotid ligation. While the incidence of multiple aneurysms is reported at $15 \%$ of all aneurysms, our experience shows a higher association between giant aneurysms and aneurysm multiplicity than previously reported.

\section{P54.}

Acute Hydrocephalus Following Aneurysmal Subarachnoid Hemorrhage (SAH)

V. Mehta, R.O. Holness, K. Connolly and S. Walling (Halifax, Nova Scotia)

We reviewed 105 cases of aneurysmal SAH seen in our unit from 1989-1993. Acute hydrocephalus was defined as a bicaudate index above the $95^{\text {th }}$ percentile for age on C.T. within 72 hrs of the ictus, $31 \%$ of the patients (33/105) developed this complication; 88\% (29/33) presented with Hunt-Hess garde III or greater. Aneurysmal location was a predictive factor, as acute hydrocephalus occurred in $88 \%$ of cases with posterior circulation aneurysms (7/8). Using the scale of Van Gijn, et al., ' we graded intraventricular and cisternal blood on CT scans. Seventy-nine percent of those with IV scores of $>4$ had acute hydrocephalus, whereas the incidence was $33 \%$ in patients with a high cisternal score but low intraventricular score (15/45).

Twenty-six percent (7/27) of survivors with acute hydrocephalus required V.P. shunting. In addition, 10 of these patients had preoperative external ventricular drainage with improvement in their grade and with no evidence of rebleeding.

Our incidence of acute aneurysmal hydrocephalus is greater than that seen in the recent co-operative study. As in the co-op study, we found that posterior circulation aneurysms are associated with a higher risk of acute hydrocephalus. Intraventricular blood was more predictive of acute hydrocephalus than cisternal blood.

The therapeutic implications of these findings will be discussed in the light of the current literature.

\footnotetext{
1Jan Van Gijn et al.: "Acute hydrocephalus after aneurysmal subarachnoid hemorrhage."
} J Neurosurg 63: 355-362 (1985). 


\section{P55. Withdrawn}

P56.

\section{Y-Aminobutyric Acid is Cytoprotective to Cerebral Ischemia in Gerbils}

\section{S. Lal, A. ShualB and S. IJaz (Saskatoon, Saskatchewan)}

Release of neurotransmitter glutamate and activation of receptor operated calcium channels may increase degree of ischemic damage. Inhibition of excitatory neurotransmitters should result in cytoprotection of ischemic brain tissue. This study evaluates effect of baclofen, an inhibitor of presynaptic glutamate release, on ischemic gerbil cortex, hippocampus (CA1 and CA4), striatum and thalamus. Double blind histological evaluation was done in 4 groups (total 36 animals): a control group (9 animals) and 3 groups (27 animals) with varying doses of baclofen. A single episode of five minutes of cerebral ischemia was used. Baclofen in doses of $0,25,50$ and $100 \mathrm{mg} / \mathrm{kg}$ were given to different groups five minutes prior to ischemic insult. This was followed by intraperitoneal injections given 24 and 48 hours after the initial insult. Statistically significant histological cytoprotection was demonstrated. Doses of $25 \mathrm{mg} / \mathrm{kg}$ demonstrated significant protection of the cortex $(p=0.0002)$, the CAl and CA4 regions of the hippocampus $(p=0.0004)$ and 0.0001$)$ respectively. The $100 \mathrm{mg} / \mathrm{kg}$ dose had the most significant protection at the striatum $(\mathrm{p}=0.0011)$, and the thalamus $(\mathrm{p}=$ 0.0008 ). All statistical comparisons were done using non-parametric tests (Mann-Whiteney U test). Our study demonstrates that baclofen is cytoprotective to ischemic neuronal cells, in particular, within the hyppocampus. Further study in inhibition of excitatory neurotransmitter release with baclofen is required.

\section{P57.}

\section{Mechanism and Duration of Neuroprotection by Cell Permeant $\mathrm{Ca}^{2+}$ Chelators in Focal Cerebral Ischemia}

M. Tymianski, I. Spigelman, L. Zhang, P.L. Carlen, C.H. Tator, M. Charlton and M.C. Wallace (Toronto, Ontario)

Cell-permenat $\mathrm{Ca}^{2+}$ chelators such as 1,2 bis-(2-aminophenoxy) ethane-N,N,N',N'-tetraacetic acid acetoxymethyl ester (BAPTA-AM) protect neurons in experimental focal cerebral ischemia. However, their in vivo actions are uncertain, and their protective efficacy proven only in brief cerebral ischemia paradigms. Here, we examine their mechanism of action in vitro and duration of efficacy in vivo. Electrophysiological studies were made in CAI neurons in rat hippocampal slices. When superfused with BAPTA-AM $(30-50 \mu \mathrm{M}), \mathrm{CA} 1$ somatic field potential recordings showed attenuation of the population spike amplitude, and intracellular recordings showed reduced exceitatory post-synaptic potentials, indicating inhibition of excitatory synaptic transmission. Also, $\mathrm{Ca}^{2+}$-dependent accommodation and post-spike train hyperpolarizations were reduced, indicating $\mathrm{Ca}^{2+}$ chelation near the internal cell membraine surface. To determine whether $\mathrm{Ca}^{2+}$ chelators reduce the size of cerebral infarction rather than simply delaying its evolution, we studied the effects of BAPTA-AM treatment on infarction size $24 \mathrm{hr}$ after permanent middle cerebral artery occlusion. Fischer rats (n $=8$ per group) were pre-treated with saline, BAPTA-AM (20 $\mathrm{mg} / \mathrm{kg})$ or $\mathrm{MK}-801(0.5 \mathrm{mg} / \mathrm{kg})$. Infarction volumes in animals treated with BAPTA-AM were reduced by $50.5 \%$ as compared with controls $(p=0.018)$, whereas animals treated with MK-801 experienced an insignificant infarct volume reduction $(26 \%, \mathrm{p}=$ 0.27 ). These data show a persistence of neuroprotection by the $\mathrm{Ca}^{2+}$ chelator at $24 \mathrm{hr}$, and indiate that it may act by attenuating synaptic transmission and sub-membrane $\mathrm{Ca}^{2+}$ excess.

P58.

Mapping of the Basal Forebrain Cholinergic System of the Dog: A Choline Acetyltransferase Immunohistochemical Study

R. St-JACQUeS, H.M. SchipPER, W. Gorczyca and G. Mohr (Montreal, Quebec)

In an effort to produce a canine model of basal forebrain focal ischemia with memory deficits, we have recently shown that dogs possess a medial striate artery that perfuses basal forebrain territory, homologous to the human recurrent artery of Heubner. Since the basal forebrain cholinergic system, implicated in cognitive functions, has never been precisely mapped in this animal, we undertook such study using choline acetyltransferase (ChAT) immunohistochemistry.

Floating $40 \mu \mathrm{m}$ thick coronal sections, from the head of the caudate nucleus to the beginning of the hippocampus, were stained for ChAT using a monoclonal antibody. Representative sections from one dog were schematically drawn using a drawing tube attached to the microscope. These outlines were used for cell density measurements, and a video analysis system was used to obtain morphometric data.

Cholinergic neurons were identified in the medial septal nucleus (Ch1) just dorsal to the anterior commissure (AC). Others were found as a continuum in the vertical (Ch2) and horizontal ( $\mathrm{Ch} 3$ ) nuclei of the diagonal band of Broca extending from $4 \mathrm{~mm}$ rostral to the $\mathrm{AC}$ for $\mathrm{Ch} 2$, to the level of the $\mathrm{AC}$ for Ch3. The fourth group of cholinergic neurons represents the nucleus basalis magnocellularis (Ch4). Located ventral to the globus pallidus, $\mathrm{Ch} 4$ extends within the substantia innominata from $2.5 \mathrm{~mm}$ rostral to $9 \mathrm{~mm}$ caudal to the $\mathrm{AC}$.

Except for the caudal component of the Ch4 population, most of this cell group is located within the perfusion territory of the medial striate arteries. The Ch4 cell group in dogs is better delineated than that of rodents but is not as sharply demarcated as in human and nonhuman primates.

P59.

High Extracellular GABA Levels in Hippocampus - As a Mechanism of Neuronal Protection in Cerebral Ischemia in Adrenalectomized Gerbils

J. Ravindran, A. Shuaib, S. IJaz, P. Galazka, R. Ishaqzay, T. WAQAR and H. MIYASHITA (Saskatoon, Saskatchewan)

Adrenalectomy protects the brain from delayed neuronal damage that occurs following transient forebrain ischemia in gerbils. Gamma amino butyric acid (GABA) and GABA mimetic drugs also have neuro-protective effect. In this study we estimated the extracellular glutamate and GABA levels in the hippocampus during transient forebrain ischemia in adrenelectomized 
gerbils $(n=9)$ compared to controls $(n=6)$. Duration of ischemia was 10 minutes and glutamate and GABA levels were measured with in vivo micordialysis. Microdialysis was started two hours after the placement of probe (to stabilize baseline) and samples were collected at 10 minute intervals. Pattern of glutamate release did not show any difference between adrenelectomized animals and controls. Adrenelectomized animals showed marked increase in GABA levels during ischemia and up to 30 minutes after ischemia $(P=<0.02$ Student $t$ test unpaired). The enhanced GABA release may be one of the possible mechanisms of neuronal protection against ischemia in adrenelectomized gerbils.

\section{P60.}

\section{Blood Brain Barrier Permeability of NBQX in Normal and} Focally Ischemic Rat Brain

M.J. Hogan, M. Sharma, A. Guedde and A.M. Hakim (Montreal, Quebec; Ottawa, Ontario)

The AMPA receptor antagonist NBQX (2,3-dihydroxy-6nitro-7-sulfamoyl-benzo $(F)$ quinoxaline) has been reported to be neuroprotective in cerebral ischemia presumably through antagonism of glutamate action at the AMPA receptor site. We undertook to measure the clearance $\left(\mathrm{K}_{1}\right)$ of systemically administered NBQX across the blood brain barrier in normal and ischemic brain. Adult male Sprague-Dawley rats with and without focal cerebral ischemia (middle cerebral + common carotid artery occlusion) were studied. $\left[{ }^{14} \mathrm{C}\right] \mathrm{NBQX}(10 \mu \mathrm{Ci}, 99$ $\mathrm{mCi} / \mathrm{mmol}$ ) was infused IV and allowed to circulate from 5 to 60 minutes before termination of studies 4 hours after onset of ischemia. Regional volumes of distribution of NBQX were then determined at the various normalized circulation times and regional $K_{1}$ estimates calculate. In the control group $(n=16)$ no regional differences in clearance of NBQX across the blood brain barrier were observed and the clearance was uniformally low, $5.7 \pm 1.6 \times 10^{-3} \mathrm{ml} / 100 \mathrm{~g} / \mathrm{min}$ for all brain regions combined. Similarly, in ischemic animals $(n=10)$ no difference was observed in regional $\mathrm{K}_{1}$ estimates between hemispheres and no regional variations in $K_{1}$ were observed. The overall clearance of NBQX from plasma to brain was $2.1 \pm 4.2 \times 10^{-3}$ $\mathrm{ml} / 100 \mathrm{~g} / \mathrm{min}$ in the ischemic group. This did not differ from the control group. We conclude that NBQX penetrates across the blood brain barrier very slowly at 4 hours following irreversible cerebral ischemia.

Supported my MRC Canada, and Heart and Stroke Foundation of Canada.

\section{P61.}

NBQX Therapy Does not Prevent Calcium Channel Activation Following Middle Cerebral Artery Occlusion in the Rat

M.J. Hogan, D. Corbett and A.M. Hakim (St. John's, Newfoundland; Ottawa, Ontario)

The AMPA receptor antagonist NBQX (2,3-dihydroxy-6nitro-7-sulfamoyl-benzo (F) quinoxaline) has been reported to be protective during cerebral ischemia. We have previously shown that in vivo binding of the L-type voltage sensitive calcium channel (VSCC) antagonist, $\left[{ }^{-3} \mathrm{H}\right]$ nimodipine, identifies cell membrane depolarization and VSCC activation in ischemic brain. These studies were performed to assess the effect of NBQX therapy on VSCC activation during acute focal cerebral ischemia produced in male Sprague-Dawley rats by simultaneous middle cerebral (MCA) and ipsilateral common carotid artery (CCA) occlusion. At 45 minutes prior to $\mathrm{MCA}+\mathrm{CCA}$ occlusion rats received either NBQX $(30 \mathrm{mg} / \mathrm{kg}$ IV bolus followed by $10 \mathrm{mg} / \mathrm{kg} / \mathrm{hour}$ IV infusion) $(\mathrm{n}=10)$ or carrier only (lithium chloride in $5 \%$ dextrose solution) $(n=10)$. $\left[{ }^{-3} \mathrm{H}\right]$ Nimodipine $(250 \mu \mathrm{Ci}, 130 \mathrm{Ci} / \mathrm{mmol})$ was infused IV 15 minutes prior to $\mathrm{MCA}+\mathrm{CCA}$ occlusion. Rats were decapitated 15 minutes after onset of ischemia and regional $\left[{ }^{-3} \mathrm{H}\right]$ nimodipine measured autoradiographically. The percentage of cerebral cortex showing increased binding in NBQX treated rats was $8.3 \pm$ $1.9 \%$ and was not statistically different from the percentage in lithium chloride controls $10.2 \pm 3.5 \%$. We conclude that pretreatment with intravenous NBQX does not prevent cell membrane depolarization or VSCC activation in acute focal cerebral ischemia.

Supported by MRC Canada, Heart and Stroke Foundation of Canada.

\section{P62.}

\section{Effects of NBQX on CA1 Necrosis and [-3H] Nimodipine Binding Following Forebrain Ischemia}

D. Corbett, F. Colbourne, M.J. Hogan and A.M. Hakim (St. John's, Newfoundland; Ottawa, Ontario)

Previous work identified two phases of increased calcium channel activation (i.e., increased $\left[{ }^{-3} \mathrm{H}\right]$ Nimodipine binding) triggered by forebrain ischemia in the rat. Widespread activation occurs within 30 minutes of ischemia, returning to preischemic levels at 2 hours. By 24 hours, Nimodipine binding rises again in vulnerable brain areas (e.g., CAl). We sought to determine if the AMPA antagonist, NBQX, which protects against moderate ischemia, would prevent both the necrosis and rise in calcium channel activation induced by severe ( 20 minutes) ischemia.

Four groups of rats were occluded under Halothane anesthesia and given NBQX (90 mg/kg, i.p.) $30 \mathrm{~min}$ prior (PRE) or 2 hours after (POST) occlusion. After 5 day survival CAl cell counts were determined (\% of normal). The 6 autoradiographic groups also received PRE of POST NBQX (or vehicle). $\left[{ }^{3} \mathrm{H}\right]$ Nimodipine was injected 30 minutes prior to sacrifice. Survival time was 30 minutes or 24 hours after ischemia. The hitological control group sustained severe injury to CA I, $14.3 \pm 13.2 \%$ (mean $\pm \mathrm{sd}$ ) neurons surviving. Pre- and post-treatment with NBQX significantly attenuated CA I cell loss $(55.2 \pm 39.4 \%, \mathrm{p}=$ $.03 ; 41.7 \pm 34.8 \%, \mathrm{p}<.01$ respectively, t-test $)$.

These results show that NBQX reduces CAl necrosis even in severe ischemia. Binding data will be discussed in relation to histological outcome at the meeting.

\section{P63.}

\section{Paraparetic-Paresthetic Transients of Carotid Artery Disease}

\section{F. VELoso (Regina, Saskatchewan)}

T.I.A. is classically divided into carotid artery distribution with ipsilateral visual disturbances and/or contralateral sensory-motor 
or speech disturbances and vertebrobasilar artery distribution with either disturbances of consciousness, equilibrium, cranial nerve function, or bilateral extremities sensory-motor function particularly of the lower extremities. We are reporting ten patients with carotid artery disease presenting with episodic transient paraparesis and/or paraparesthesia simulating vertebrobasilar artery T.I.A. We recommend that paraparetic-paresthetic transients mimicking basilar artery T.I.A. resulting from carotid artery disease be managed similarly as if the patient is presenting with symptomatic carotid artery disease.

\section{P64.}

\section{Correlation of Neuronal Injury with Quantitative T1 and T2 MRI in a Rat Model of Forebrain Ischemia}

J. Peeling, R.J. Buist, S. Sunagawa, B. Psooy and G.R. Sutherland (Winnipeg, Manitoba; Calgary, Alberta)

Following forebrain ischemia (12 min carotid occlusion plus hypotension) in rat, accurate T1 and T2 MRI values were obtained at progressing times for up to 21 days, and correlated with the degree of injury in selectively vulnerable brain regions. No significant changes were observed in $\mathrm{T} 1$ values following ischemia. T2 did not change before $12 \mathrm{hr}$ post-ischemia, when $\mathrm{T} 2$ in the dorsolateral striatum began to increase, coincident with the appearance of histologically defined neuronal injury in this region. $\mathrm{T} 2$ then increased until day 2 post-ischemia, then began to decrease, falling significantly below base-line by day 7 . In the hippocampus, $\mathrm{T} 2$ did not change until day 2 , when it increased markedly, coincident with the appearance of significant neuronal injury. Thereafter the $\mathrm{T} 2$ value decreased slowly until day 14, when it was significantly less than preischemia. The increase in $\mathrm{T} 2$ is probably due to increasing edema. The decrease in $\mathrm{T} 2$ below normal may reflect a glial response. The results do not encourage the use of $\mathrm{T} 1$ or $\mathrm{T} 2$ weighted MR images as early predictors of outcome following global cerebral ischemia. However the results confirm that developing injury can be monitored with $\mathrm{T} 2$ weighted imaging.

\section{P65.}

\section{A New Experimental Method for Local Cooling of the Brain}

R. Mazagri, A. Hedayat, A. Shuaib and M. Khan (Saskatoon, Saskatchewan)

It has been known for years that hypothermia has a protective effect on the brain during ischemia, and prevents neuronal damage. Methods in the past to cool the brain have utilized cooling of the entire body. This is almost impractical in a conscious patient and more importantly is usually associated with metabolic disturbances.

In an attempt to further develop a method of cooling the brain selectively, we report a new technique in rabbits. Adult male rabbits were anaesthesized and 4 burr holes were made on both sides of the cranium, then cold Helium gas was passed under low pressure sub-durally through one burr hole. Brain and body temperature were monitored throughout the procedure.

The brain temperature was $37^{\circ} \mathrm{C}$ before starting the cooling and decreased to $32^{\circ} \mathrm{C}$ within $45-60$ minutes after starting the procedure. There were no complications. Selected hypothermia of the brain by the use of cold Helium gas may offer an alternative method for brain protection during surgery.

Details will be discussed.

P66.

Efficacy of Antioxidant LY231617 by Various Treatments on 5 Hour Old Focal Ischemia Assessed by TTC and LCGU

\section{Y.I. ITo and Y.L. YAMAMOTo (Montreal, Quebec)}

Previous study of treatment of retrograde perfusion of the cerebral vein (RPCV) with antioxidant LY231617 on the 5 hour old focal ischemia improved LCBF in both cortical and striatum areas. In this study, the efficacy of the treatment and viability of ischemic tissue were assessed by triphenyltetrazolium chloride (TTC) stain and autoradiographic local cerebral glucose utilization (LCGU).

Method: Focal ischemia of SD rats was produced by middle cerebral artery occlusion (MCAO) with coagulation of all perforating branches. Group A with intravenous administration of LY231617 (10 mg/kg/2hr); Group B, RPCV with saline, Group C, RPCV with LY231617 (10 mg/kg/2hr). The treatment was started 5 hours after MCAO for 2 hour periods.

Result: In Group C, TTC stain showed significant characteristic reduction of ischemic damage in the caudate (over $91 \%$ ) and cortical (over 76\%) areas as compared with Groups $A$ and B. LCGU of Group C were well preserved in the striatum, but not in Groups A and B.

Conclusion: The treatment of RPCV with LY231617 showed a unique amelioration of ischemic damages not only in cortical area but also in striatum area in the 5 hour old focal ischemia due to rich collateral flow between superficial and deep cerebral veins at the microcirculatory level.

\section{P67.}

The Effect of Mild Hypothermia on Cerebral Energy Metabolism During and Following Hypoxic-Ischemic Brain Damage in the Immature Rat

\section{J.Y. YAGER and M. SHARGOOL (Saskatoon, Saskatchewan)}

We have previously shown that mild hypothermia (34 \& $31^{\circ} \mathrm{C}$ ) protects the immature brain during but not following $\mathrm{H}-\mathrm{I}$ (Ped Res 34: 525-529, 1993). To determine the effect of temperature on cerebral high-energy phosphate reserves during the evolution and recovery from H-I, groups of 7-day rat pups underwent unilateral common carotid artery ligation and exposure to hypoxia in $8 \%$ oxygen at either 37,34 , or $31^{\circ} \mathrm{C}$ for 3.0 hrs. Other groups were exposed to $\mathrm{H}-\mathrm{I}$ at $37^{\circ} \mathrm{C}$ but recovered at either 37,34 , or $31^{\circ} \mathrm{C}$. Control animals in each of the 3 temperature groups were maintained in $21 \%$ oxygen for $180 \mathrm{~min}$. At specific times both during and following $\mathrm{H}-\mathrm{I}$, brains of individual rat pups (6-8) were rapidly frozen in liquid nitrogen to preserve high-energy phosphates. $31^{\circ} \mathrm{C}$ preserved normal brain ATP $(2.23$ $\mathrm{mmol} / \mathrm{kg})$ for up to $180 \mathrm{~min}$ of $\mathrm{H}-\mathrm{I}$, compared $34^{\circ} \mathrm{C}(0.47$ $\mathrm{mmol} / \mathrm{kg})$ or $37^{\circ} \mathrm{C}(0.59 \mathrm{mmol} / \mathrm{kg})(\mathrm{p}<.001)$. Despite marked differences in the extent of brain damage during $\mathrm{H}-\mathrm{I}$ at 34 and $37^{\circ} \mathrm{C}$, no difference in ATP concentrations between the 2 groups was shown. ATP restoration was not significantly different in 
any of the 3 groups recovered at 37,34 , or $31^{\circ} \mathrm{C}$. Thus, hypothermia of $31^{\circ} \mathrm{C}$ during $\mathrm{H}-\mathrm{I}$ preserves high-energy phosphate compounds; whereas lesser degrees of hypothermia did not. Mechanisms other than a reduction in cerebral metabolism may be responsible for the benefits of mild hypothermia during H-I. Post H-I hypothermia was ineffective in restoring ATP.

\section{P68.}

\section{Protective Effects of Ketone Bodies on Hypoxic-Ischemic Brain Damage in the Immature Rat}

\section{J.Y. YAGER and M. SHARGOOL (Saskatoon, Saskatchewan)}

Fasting markedly protects both the adult and newborn brain from hypoxic-ischemic (H-I) damage. In adults, the presumed mechanism is a reduction in glycolytic lactate production. To determine the mechanism underlying the beneficial effect of fasting in the immature animal; 7-day rat pups underwent unilateral common carotid artery ligation and $\mathrm{H}-\mathrm{I}$ induced by placing groups of rat pups (4/jar) in $500 \mathrm{ml}$ glass jars through which $8 \%$ oxygen/nitrogen was vented via inlet/outlet portals. Temperature was maintained by partially submerging the jars in a $37^{\circ} \mathrm{C}$ waterbath. Pups fasted for 12 hrs were compared with controls, and those which received $0.1 \mathrm{ml}$ subcutaneous injections of either 2,5 or $10 \mathrm{mmol} / \mathrm{kg}$ of beta-hydroxybutyrate. Following $\mathrm{H}-\mathrm{I}$, rat pups were allowed to recover with their dams to 30 days of age after which time brains were assessed neuropathologically and ranked according to the severity of damage. In separate groups of rat pups, blood glucose, lactate, and beta-hydroxybutyrate was measured prior to and at the end of H-I. Fasting, as well as supplementation of 5 or $10 \mathrm{mmol} / \mathrm{kg}$ beta-hydroxybutyrate was significantly protective compared to controls ( $\mathrm{p}<$ 0.05 ), despite higher lactate concentrations in the latter 2 groups. In the immature animal, the protective affect of fasting may be secondary to the induced hyperketonemia and, is not the result of a reduction in lactate production, as it presumed in the adult. Utilization of substrates other than glucose in the sick premature infant may reduce the risks of subsequent ischemic brain damage.

\section{P69.}

\section{Short-lasting Focal Cerebral Ischemia Raises CBF During a Subsequent Episode}

\section{K. Matsushima and A. Hakim (Ottawa, Ontario)}

We have explored one mechanism by which a short episode of "tolerable" ischemia may protect the brain during a subsequent more severe ischemic insult. One day prior to the ischemia, a platinum electrode was implanted in the cortex supplied by the middle cerebral artery (MCA) to allow measurement of cerebral blood flow (CBF) by the $\mathrm{H}_{2}$-clearance method. In group $1(n=9)$, rats underwent permanent occlusion of the left common carotid (CCA) and external carotid (ECA) arteries under general anesthesia. This was followed by advancing a 4-0 suture into the left MCA. The suture was withdrawn $30 \mathrm{~min}$ later. Four days later, the MCA occlusion was repeated and maintained for $180 \mathrm{~min}$. CBF measurements were obtained at various times and compared with group $2(n=8)$ which was spared the first 30 min period of MCA occlusion but was otherwise treated identically. $\mathrm{CBF}$ in the two groups was comparable except during the $180 \mathrm{~min}$ ischemic episode where it remained significantly higher in group 1 (mean $\pm S D, C B F=37.5 \pm 1.9$ $\mathrm{ml} / 100 \mathrm{~g} / \mathrm{min})$ than in group $2(31.9 \pm 3.6, \mathrm{p}=0.026)$. Our data suggest that a prior ischemic episode elevates CBF and may raise the threshold for infarction.

P70.

Delayed Atrophy of Basal Ganglia Nuclei Following Localized Lesions of the Cerebral Cortex: Implication for Stroke

M.F. MazureK, E. Kiriakopoulos, S. Furtado, S. Garside and J. Woulfe (Hamilton, Ontario; Calgary, Alberta)

Little is known about the effects of stroke on distant, noninfarcted, parts of the brain. We have begun to study this issue by examining the effects of long-standing lesions of the cerebral cortex on subcortical nuclei. Animals received excitotoxic or aspirative lesions of the frontal cortex, 15 months prior to sacrifice. Frozen transverse sections of the forebrain and brain stem were cut, and adjacent sections were processed immunohistochemically for tyrosine hydroxylase (TH). Substance P (SP) or NADPH-diaphorase, while a fourth series of sections was Nissl stained to delineate architectonic boundaries. Following cortical lesions, there were marked atrophic changes in the ipsilateral substantia nigra (SN) and striatum. Histological analysis of the shrunken $\mathrm{SN}$ showed a depletion of $\mathrm{TH}$-immunoreative neurons, and reduced intensity of SP fiber staining. Within the atrophic striatum, there was a significant decrease in the number of SP-immunoreactive spiny projection neurons, with normal or increased numbers of NADPH-diaphorase-stained aspiny cells. These results indicate that chronic lesions of the cerebral cortex can produce atrophic changes in distant subcortical nuclei, including SN and striatum. These subcortical changes may have functional consequences for patients who have suffered strokes involving the cerebral cortex.

P71.

\section{The Study of the Three Sensory Evoked Potentials in} Patients with Cerebral Infarct

\section{L.-Y. FenG, Y.-F. HE and K. FAN (Shijiazhuang, P.R. China)}

According to the measured value of three SEPs for 20 normal individuals, a study was made of the SEPs for 59 patients with cerebral infarct. Of them, 34 men and 25 women with a mean age of $57.8+8$, all demonstrated by $\mathrm{CT}$.

We found that 45 of the 59 patients had abnormal trigeminal SEP(TSEP), 48 had abnormal median SEP(MSEP), and 51 had abnormal tibial SEP(TiSEP), the sensitivity being 76.3\%, $81.3 \%$ and $86.4 \%$ respectively. Both TSEP and MSEP together in 53 of all the patients, both TSEP and TiSEP in 55, both MSEP and TiSEP in 56, and all three SEPs together in 29 of the patients were abnormal, the sensitivity being $89.8 \%, 93.2 \%, 94.9 \%$ and $49.2 \%$. But any one kind of the three SEPs in 58 was abnormal, the sensitivity being $98 \%$. 
Our results showed that any one kind of the SEPs is not assuredly the sign of all the lesions of the sensory pathway. Although any two SEPs are good for the patient, there are some false negative ratios. Only when all three SEPs together are tested, they are relately more ideal, that may be more helpful in evaluating the extent and degree of the actual lesions in them. So, this result indicates all three SEPs together are the best methods for the clinical and electrophysiologic study of patients with cerebral infarct or other patients with corticospinal involvement.

\section{P72.}

\section{Cervical Radiculopathy as a Manifestation of Giant Cell Arteritis}

\section{Rivest, D. Brunet, R. Desbiens and J.P. Bouchard} (Quebec, Quebec)

Giant cell arteritis may present with a number of neurological clinical pictures such as ischemic optic neuritis or focal brain ischemic events. We report a patient with a typical C-5 radiculopathy due to this cause, a rare occurrence according to the literature (Sanchez et al., 1983).

A 53-year-old female presented with left atypical facial pain (increased by masticatory activity), posterior cervicalgia, fatigue and nocturnal sudation. No visual or focal neurological symptoms were found. Sedimentation rate (SR) was $83 \mathrm{~mm} / \mathrm{h}$.

Two weeks later, the patient complained of acute pain in the left shoulder and arm, followed a week later by complete paralysis of shoulder abduction and external rotation, as well as flexion of the forearm. Hypoesthesia was found on the left shoulder crest (C4). EMG revealed increased insertional activity and rare fibrillations in the left deltoid, infraspinatus and biceps muscles. Spinal fluid, cervical myelogram and CT scan were normal, but $\mathrm{SR}$ was 92 . Angiography revealed a re-permeated aspect of the left vertebral artery. Giant cell arteritis was confirmed on biopsy of one temporal artery. Systemic symptoms rapidly vanished with corticosteroids and motor function of the left upper limb was good at the two month follow-up.

\section{P73.}

\section{Conjugal Patent Foramen Ovale and Stroke}

J.-P. Bouchard, M. Petitelere, R. Desbiens, C. Nadeau, G. Houde, S. Tremblay and D. Simard (Quebec, Quebec)

According to the literature transesophageal echocardiography (TEE) improves by threefold ( $14 \%$ to $41 \%$ ) the chances to identify a cardiac embolic source, as compared to trans-thoracic echo (TTE). We report similar TEE findings in a husband and his wife, who both suffered ischemic events.

Case reports: (1) A 54-year-old woman was evaluated for repeated short episodes of left face and arm numbness. Brain MRI, carotid Doppler and TTE were normal. An aneurysm of the foramen ovale was seen with TEE. With contrast medium there was a discrete right-left shunt. The patient has remained free of TIA under Warfarin.

(2) Her husband, at 56 years old, was admitted in 1993 for an episode of numbness of the left side of the body. He had been treated in 1986 for intracerebral hemorrhage in the left frontal lobe. The left middle cerebral artery was then occluded proximally. TTE was normal, but TEE showed an aneurysm of the foramen ovale and a discrete left-right shunt.

Patent foramen ovale is probably a more frequent source of cerebral embolism than previously believed. The significance of such a finding on TEE, however, has yet to be established.

P74.

\section{Iron Deficiency Anemia: A Frequent Cause of Transient Neurologic Deficit/Stroke in Children?}

J.Y. Yager, N. Lowry and A. WiLfong (Saskatoon, Saskatchewan)

Iron deficiency has been implicated in neurologic abnormalities ranging from irritability to pseudotumor cerebri. Infrequently, transient neurologic deficits have been documented. We report 4 children, each presenting with transient ischemia and/or stroke in whom iron deficiency and thrombocytosis were responsible. The following 2 brief summaries will serve as examples:

Patient TM: $5 \mathrm{mth}$ male admitted with generalized status epilepticus following a 2 day history of fever and vomiting. Septic work-up was negative. Laboratory revealed a microcytic anemia Hgb (4.5), Plts (1082 × 109/L), Iron (9) and TIBS (61), $\%$ Saturation $(2 \%)$, secondary to chronic goat's milk ingestion. MRI scan showed superior sagittal and straight sinus thrombosis, and bilateral basal gangliar hemorrhages.

Patient SL: $11.5 \mathrm{mth}$ female presented with a mild left hemiparesis of acute onset following a 3 day hisotry of an upper respiratory tract infection. Work-up revealed a Hgb (109), Plt (693 $\times 10^{9} / \mathrm{L}$ ), Iron (4) and TIBC (58), \%Sat. (7). CT scan showed a $2 \mathrm{~cm}$ right basal gangliar infarct and extension into the internal capsule. None of the 4 children to be presented had evidence of an underlying hypercoagulable state.

Iron deficiency anemia and thrombocytosis should be considered more frequently as a cause of transient/permanent neurologic deficits in children; its presence often complicating otherwise benign illness.

P75.

\section{Postoperative Stroke in Coronary Artery Bypass Graft} Surgery: Racial Differences

M. Assaf, K. Gaines and R. van der ZwaAG (Memphis, Tennessee)

Background and Objective: Racial differences in stroke prevalence and mortality have been reported. We conducted a retrospective study to determine the racial differences in post CABG surgery stroke rate, mortality and risk factors.

Methods: All patients who underwent isolated CABG surgery with and without associated diagnosis of post $\mathrm{CABG}$ stroke (from October 1984 to July 1992) were identified by computer search. Medical records and neuroimaging studies of patients with post CABG stroke were reviewed and 23 pre, peri and post operative risk factors were evaluated.

Results: 6603 patients underwent isolated $\mathrm{CABG}$ surgery, $6095(92.3 \%)$ whites and $508(7.7 \%)$ blacks. $128(1.93 \%)$ had 
acquired acute stroke. The post CABG stroke rate in whites was 1.96 (120 patients), $79 \mathrm{M}, 41 \mathrm{~F}$, age $45-88$ (mean 69.3 years) and in blacks $1.57 \%$ (8 patients), $3 \mathrm{M}, 5 \mathrm{~F}$, age 62-77 (mean 70.8 years). The mortality rate for those who sustained stroke was $29.7 \%$ (38 patients). 35 deaths occurred in whites $(29.2 \%$ ), 25 males and 10 females, compared to 3 deaths in blacks $(37.5 \%)$, all females. None of the 23 risk factors differed significantly by race.

Conclusion: This study failed to prove any significant racial differences in post CABG stroke rate, mortality and risk factors.

P76.

Top of Basilar Artery Syndrome and Thalamic Aphasia; A Case Report

F. Tanha, J. Donat, A. Shuaib, A. Kirk and I. Gulka (Saskatoon, Saskatchewan; London, Ontario)

Patients with midbrain infarction are usually comatose and have a poor prognosis. We report a case of Top of Basilar Artery Syndrome in an eighty-four-year-old right-handed man with MRI-Documented infarction of medial basal thalamus, superior colliculus, pretectal area, medial longitudinal fasciculus, occulomotor nucleus, and left occipital lobe. The clinical manifestation included right homonomous hemianopsia, bilateral occulomotor palsy, spastic dysarthria, and thalamic aphasia which correlated with radiological findings. The presentation is unusual because of the extent of involvement in a fairly alert patient with a "pseudo coma" pattern due to bilateral ptosis and thalamic aphasia.

P77.

\section{Interictal Radial and Horizontal Neglect and Ictal "Antineglect"}

\section{C.A.J. BoYLE and A. KIRK (Saskatoon, Saskatchewan)}

Hemispatial neglect is commonly the result of tumor or stroke, but Heilman and Howell reported seizure-induced neglect in a patient with right parieto-occipital seizures. Their patient had ictal neglect causing changes in horizontal line bisection. We report a 46-year-old man who was observed for neglect both interictally and ictally during simple partial seizures from a right temporo-parietal focus, at the site of resection of an astrocytoma 13 years previously. He was hemianopic but had neither auditory nor tactile extinction. The patient was presented with line bisection tasks, between and during clinical seizures. Interictally, he bisected horizontal lines to the right of midpoint, and radial lines distal to midpoint. During seizures, he consistently bisected horizontal lines far to the left and radial line proximal to midpoint. In fact, during seizures he usually attempted to make a mark far to the left and far proximal to the page making measurement difficult. Following medical control of his seizures he was much more accurate at line bisection tasks. This case illustrates seizure-induced neglect occurring in more than one plane.

\section{II-3. NEURO-ONCOLOGY}

P78.

Human Monoclonal Antibody BT32/A6 Recognizes a Cell Cycle-Independent Neuroectodermal Tumor-Associated Surface Antigen

M.D. Dan, E.M. Earley, M. Griffin, P.K. Maitl, A. Prashar, X.Y. Yuan, A.D. FriesEN AND H.A. KaPlan (New Orleans, Louisiana; Winnipeg, Manitoba)

The human IgM monoclonal antibody BT32/A6, derived from a peripheral blood lymphocyte of a 14-year-old boy with von Recklinghausen's disease and an intraventricular low grade astrocytoma, labels a cell surface antigen on viable, clonogenic cells of SK-MG-1, a human glioma cell line. Approximately $100 \%$ of live SK-MG- 1 cells express the BT32/A6 antigen by flow cytometry. Labelling of SK-MG-1 by BT32/A6 was shown to be independent of: 1) cell growth rate, 2) viability, and 3) cell cycle state. Antibody BT32/A6 binds complement and reacts with at least one other glioma, melanoma, and neuroblastoma cell line, but not with a colon cancer line. Specific immunohistochemical staining of tumor cell lines and paraffin-embedded tissue sections (but not normal brain) was demonstrated. The recognition of a tumor-specific, cell cycle-independent, surface antigen by BT32/A6 makes it a promising candidate for adjunctive therapy in neuro-oncology because conventional therapies (cytoreduction, radiotherapy, chemotherapy) are directed primarily at proliferating malignant cells, whereas the antigen recognized by BT32/A6 is expressed on both proliferating and non-proliferating malignant cells, but not on normal brain.

\section{P79.}

\section{Results of Radiosurgery for Recurrent Malignant Gliomas:} UCSF Experience 1991-1993

M.W. McDermott, P.K. Sneed, M.D. Prados, D.A. Larson, W.M. Wara, M.S.B. EdWards and Philip H. Gutin (San Francisco, California)

Between 9/18/91 and 11/1/93 we explored the effectiveness of radiosurgery in the treatment of 60 patients with recurrent gliomas. All treatments were carried out on a gamma knife radiosurgical unit. Four patients had 2 lesions treated and 3 patients were re-treated for the same lesion. Original histologic diagnoses were: glioblastoma $=33$; anaplastic astrocytoma $=9$; mixed glioma $=6$; low grade astrocytoma $=5$; oligodendroglioma $=2 ;$ malignant ependymoma $=3 ;$ ependymoma $=1$ and $\mathrm{JPA}=1$. The mean $/$ median volumes of treatment were $13.41 / 10.7 \mathrm{cc}$. The mean/median number of isocenters required for tumor coverage were $7.4 / 8$ and in all but 4 patients the treatment was prescribed at the $50 \%$ isodose line (range $40-90 \%$ ). Mean/median doses to the margin of the contrast enhancing lesion were $1637.1 \mathrm{cGy} / 1600 \mathrm{cGy}$, with a range of $1000-2200$ $\mathrm{cGy}$, and to the center $3257.9 \mathrm{cGy} / 3200 \mathrm{cGy}$ with a range of $2000-4400$ cGy. Follow-up after radiosurgery was available through $1 / 18 / 94$ with a mean follow-up of 8.0 months. The median time to tumor progression was 5.04 mo and the median survival $12.89 \mathrm{mo}$. While these early results are encouraging, longer follow-up is required to further evaluate the efficacy and side-effects of this form of treatment. Follow-up through 1/94, 
patterns of failure and cost and survival analysis compared to brachytherapy will be presented.

P80.

\section{Dislocation of the Pituitary Gland by Sphenocavernous Meningiomas}

F. DeMonTe and O. Al-MefTy (Houston, Texas; Little Rock, Arkansas)

From an operative series of 19 consecutive patients with cavernous sinus meningomas, 7 tumors could be subclassified as sphenocavernous meningiomas due to the significant amount of tumor extension into the sphenoid sinus. At the time of surgery, dislocation of the pituitary gland form the sella into the suprasellar cistern was observed in 5 of 7 of these patients. Initially, this was confirmed by biopsy in 2 patients, but recognized and the gland avoided in the rest. Four of the 5 patients with pituitary dislocation had evidence of hypopituitarism, whereas there was no evidence of this condition in the 2 patients with sphenocavernous meningiomas without pituitary dislocation. Pituitary dislocation in these patients is likely due to the physical dislocation of the pituitary gland from the sella, through an incompetent diaphragma sella, by the tumorous extension in the sphenoid sinus. The extension of a cavernous sinus meningioma into the sphenoid sinus, and specifically the sella turcica, may be via the intercavernous venous sinuses. Preoperative MRI did not identify the occurrence of pituitary dislocation as the gland was usually "lost" within the tumor. The possibility of pituitary dislocation in patients with sphenocavernous meningiomas, especially those with panhypopituitarism, should be considered and searched for at the time of surgery.

P81.

\section{Pseudotumor of the Cavernous Sinus}

\section{M.D. Cusimano, R. Perrin and M. Ohi (Toronto, Ontario)}

A 69-year-old woman was referred to our centre because of a cavernous sinus tumor. Thirteen years ago she developed diplopia on right gaze and three years ago developed right trigeminal neuralgia. On exam she had right $V_{3}$ and 6th nerve palsies.

CT scan was initially reported as showing a lytic process involving the right lateral aspect of the sphenoid with an associated soft tissue lesion in the cavernous sinus. MRI showed a nonenhancing soft tissue anomaly in the cavernous sinus felt to be a metastasis or meningioma. Thin section CT scanning and angiography demonstrated that the internal carotid artery was markedly ectatic and that the "lesion" was really the venous plexus of the cavernous sinus without the normally positioned carotid artery.

The patient has been followed for $1 \frac{1}{2}$ years with stabilization of her symptoms and no changes on serial imaging.

This case broadens the differential diagnosis of cavernous sinus tumors. It also highlights the importance of detailed radiological investigation and tissue diagnosis in any series where conclusions regarding the effectiveness of a specific therapy (e.g., LINAC, gamma knife) are made.
P82.

A Technique of Fractionated Stereotactic Radiotherapy for the Treatment of Intracranial Tumors

\author{
J.-L. Caron, E. Podgorsak, L. Souhami, M. Pla, B. Clark, C. \\ PLA and P. CADMAN (Montreal, Quebec)
}

Purpose: The excellent treatment results obtained with traditional radiosurgery have stimulated attempts to broaden the range of intracranial disorders treated with radiosurgical techniques. From our knowledge of biological responses to ionizing radiation, the treatment of certain intracranial tumors may benefit from a fractionation of the dose of radiation delivered. The possibility of using a focused beam hitting the chosen target and sparing surrounding tissue could make the treatment more effective and less toxic. The technique of fractionated stereotactic radiotherapy was therefore developed.

Method: The difficulty with fractionated stereotactic radiotherapy is in reproducing the precision of single fraction radiosurgery on repeated occasions. We developed a precise irradiation technique based on an "in-house-built" stereotactic frame which is left attached to patient's skull for the duration of the fractionated regimen which may vary between 6 and 12 days. Target stereotactic coordinates are marked on a Lucite box which is replaced on the frame at each treatment fraction. The target localization is verified by CT scanning using radioopaque markers on the Lucite box and small tattooed dots on the patients skin.

Conclusion: This technique is inexpensive and highly accurate. The frame is well tolerated and easily left affixed to the head of most age groups to which this technique could be applied.

P83.

Pre-operative MRI Findings in Acoustic Neuroma Surgery: Topographical and Pathological Correlations

C. Dureza, G. Mohr, J.-J. Dufour, N. Just and S. Entis (Montreal, Quebec)

MRI has become indispensable in the preoperative diagnosis of acoustic neuromas. The purpose of this study is to retrospectively analyze the predictive value of the preoperative MRI on the intraoperative topographical and pathological findings, in particular, the degree of difficulty of the tumor dissection from the brain stem and the extent of drilling of the posterior lip of the internal auditory meatus.

A total of 45 patients with pre-operative MRIs were studied. The lesions were analyzed by making several measurements in the coronal, transverse and petrous plane using fine calipers. The extent of intracanalicular growth was also measured. Signal intensity both within and around the tumor was noted, as well as degree of enhancement with gadolinium.

From this analysis, it is concluded that the probability of cochlear nerve preservation does not depend solely on degree of intracanalicular extension seen on MRI. Additionally, flow void signals surrounding the tumor can help the surgeon anticipate problematic vascular structures. It was also evident that seventh 
nerve involvement was more likely in tumors extending posterior to the axis of the intracanalicular canal on the axial images. Finally signal intensity in MRI on gadolinium enhancement, while helpful, had very little correlation with the Antoni classification.

\section{P84.}

\section{Acromegaly with Normal Growth Hormone Levels: A Case} Report

Z.-P. Chen, G. Mohr, M. SChweitzer and F. Robert (Montreal, Quebec)

The most common cause of acromegaly is excess of growth hormone $(\mathrm{GH})$ secretion. We report a very rare case of 42-yearold male patient, who had become acromegalic over the past 5 years. There were no visual changes or change in sexual function, no gynaecomastia or galactorrhoea. Both CT and MRI scans showed a large mass measuring $2.5 \times 2.5 \times 3.5 \mathrm{~cm}$, originating from the sella turcica and extending into and totally filling up the sphenoid sinus with diffusely invasive features. Basal serum GH level was within normal range, but IGF-1 was elevated with slight increased PRL and impaired GH secretory regulation as well. A pituitary adenoma pathologically confirmed was removed through transphenoid microsurgery. Immunocytochemistry study of the tumour showed only positively for PRL but no GH. We proposed that IGF-1 may be one of the inhibitors of GH and both IGF-1 and PRL might be the cause of the clinical feature of acromegaly in this patient.

P85.

Three Patients with Cholesterol Granuloma of the Petrous Apex: MRI Findings and Surgical Approach

\section{Dureza and J.-G. Villemure (Montreal, Quebec)}

Cholesterol granuloma of the petrous apex is an uncommon entity in neurosurgery. There are however certain features of this lesion both in clinical presentation and radiological characteristics that separate this from other lesions of this area.

The following three cases were determined histologically from the operative specimens to be cholesterol granulomas. In each case MRI images were obtained in sagittal, axial, and coronal planes using standard techniques. Gadolinium was also used. Prior to the MRI, a CT scan with and without contrast was performed. The adjacent vascular anatomy was visualized using digital subtraction angiography of the internal and external carotid arteries on the side of the lesion. The findings on the MRI proved to be of benefit in elucidating the nature of the tumor, while together with the CT scan and angiography determined the best surgical approach preoperatively. In all cases we opted to perform a subtemporal extradural approach. While a thorough evacuation of the lesion was performed the capsule was left intact.

The purpose of this study is to review the MRI findings of this lesion and to discuss the benefits of the surgical approach utilized in all three cases in light of the natural history of cholesterol granulomas and with respect to the anatomy of the middle cranial fossa.
P86.

\section{Effective Combination Therapy for Radiotherapy and Tamoxifen Resistant, High Grade Astrocytoma}

A. Langleben, M. Preul, D. Tampieri, A. Tsatoumas, J-P. Bahary, G. Shenouda, G. Baltuch and J.-G. Villemure (Montreal, Quebec)

Patients with anaplastic astrocytoma (AA) or glioblastoma multiforme (GM), recurrent after maximal radiotherapy and/or surgery, were initially treated on an institutional protocol of Tamoxifen (TAM) $120 \mathrm{mg} / \mathrm{m}^{2}$ b.i.d. Unfortunately, nine patients progressed; performance status (PS) deteriorated in all; CT scans showed increased size and enhancement. Because of the suggestion of increased enhancement, TAM was continued; concurrent BCNU $240 \mathrm{mg} / \mathrm{m}^{2}$ q 8 weeks was initiated. Durable clinical responses, with significant PS improvement, and CT scan responses are produced. Serial MR Spectroscopic imaging documented persistent elevation of lactate and lipid resonances in non-responders, compatible with persistent tumor viability. In responders, lactate and lipid resonances increase transiently (compatible with a shift to anaerobic metabolism and cell membrane degradation) and then decrease significantly within two months of BCNU initiation (compatible with removal of cellular debris). In some patients, remission duration equals or exceeds that of first remission.

\begin{tabular}{llllllllll}
\hline Patient & A & B & C & D & E & F & G & H & I \\
\hline Grade & GM & AA & AA & GM & GM & AA & GM & GM & GM \\
$\begin{array}{l}\text { Age 45 } \\
\text { Sex F }\end{array}$ & 42 & 42 & 56 & 43 & 41 & 43 & 44 & 48 & \\
$\begin{array}{c}\text { Duration 1st } \\
\text { remission }\end{array}$ & M & M & M & M & F & M & F & M & \\
$\begin{array}{l}\text { Month to } \\
\text { progression (prog.) }\end{array}$ & 2 & 7 & 2 & 1 & 2 & 1 & $1 / 2$ & 1 & $1 / 2$ \\
$\begin{array}{c}\text { on TAM alone } \\
\text { Radiologic response } \\
\text { to TAM/BCNU (T/B) }\end{array}$ & Prog. & MR & MR & Prog. & PR & PR & MR & Prog. SD \\
$\begin{array}{c}\text { Clinical response } \\
\text { to TAM/BCNU }\end{array}$ & none & excel- & mod- & none & stable & excel- & excel- & none. mod- \\
$\begin{array}{c}\text { Response duration } \\
\begin{array}{l}\text { T/B) } \\
\text { Survival (months) }\end{array}\end{array}$ & 0 & $12+$ & $11+$ & 0 & $8+$ & $8+$ & $8+$ & 0 & $5+$ \\
\hline
\end{tabular}

P87.

\section{Brown Tumor of the Skull Base}

M.D. Cusimano, W. Singer, R. Marotta, J. Davidson, J. BILBAO and W.S. TUCKER (Toronto, Ontario)

Brown tumors are expansile osteolytic lesions of bone occurring in hyperparathyroidism. They occur most commonly inprimary hyperparathyroidism and classically occur in the extremities. Although these tumors have very rarely been noted in the mandible, and orbit, none of the skull base have been reported.

We report the case of a 38-year-old woman who presented with a skull base lesion initially diagnosed as a meningioma who was diagnosed as having a brown tumor of the skull base. Removal of a 4.2 gram parathyroid adenoma has led to resolution of the patient's symptoms of intracranial hypertension and calcification of the lesion. 
This is the first such case report and emphasizes the clinical, radiological and pathological correlations.

\section{P88.}

The Use of the Ki-67 Antigen MIB 1 for Determining the Proliferation Index of Meningiomas, and its Comparison to the BUdR Labelling Index

\section{F. DEMonTE and L. LANGFORD (Houston, Texas)}

The 30 patients in this study underwent resection of a meningioma at the University of Texas MD Anderson Cancer Center. All tumor specimens were labelled and stained for both bromodeoxyuridine (BUdR) and the Ki-67 antigen MIB I. The BUdR labelling indices ranged from $0 \%$ to $5.8 \%$, whereas the MIB 1 labelling indices ranged from $2.24 \%$ to $19.25 \%$. The higher labelling indices obtained with MIB 1 are consistent with the labelling of cells in all but the $G_{o}$ phase of the cell cycle. BUdR, in contrast, labels only those cells in the $S$ phase of the cell cycle. There was good correlation between the BUdR and MIB 1 labelling indices $(r=0.86)$. The advantages of using the MIB 1 labelling index are: (1) greater technical ease, (2) no need to administer drug to the patient, (3) allows use on archival, decalcified and consultative material, and (4) lower cost.

\section{P89.}

\section{Teratoma Arising Within Lipomyelomeningocele in Two Patients}

M. Mikhail, G.P. Murray, F.B. Maroun, B. Cramer, C. Pushpanathan and D. Price (St. John's, Newfoundland)

The authors described two infants who presented at birth with the typical clinical features of lipomyelomeningocele. At surgery, both were found to contain Teratomatous elements within the lipomyelomeningocele. Rarely has the association of these two lesions in a single patient been reported.

Clinical features and operative findings are discussed along with literature review.

\section{P90.}

\section{A Solitary Eosinophilic Granuloma of the Frontal Lobe: Case Report}

F.B. Maroun, J.C. Jacob, A. Davis, E. Walsh, G. Murray and C. Pushranathan (St. John's, Newfoundland)

Primary intracranial eosinophilic granuloma are extremely rare. They ususally involve the hypothalmus. We reported a rare case of a primary frontal lobe eosinophilic granuloma in a 13year-old boy. A clinical radiological and pathological finding will be presented together with review of the literature.
P91.

Sub Periosteal, Sub Periorbital Dissection and Division of the Anterior and Posterior Ethmoidal Arteries for Meningiomas of the Cribriform Plate and Planum Sphenoidale

M.W. McDermott, J. Rootman and F.A. Durity (San Francisco, California; Vancouver, British Columbia)

Standard neurosurgical technique for the removal of meningiomas of the cribriform plate and planum sphenoidale, involves bifrontal craniotomy for exposure and interruption of the blood supply to tumor by removing tumor and/or dura from the floor of the anterior cranial base. In the process the blood supply from the anterior and posterior ethmoidal arteries is interrupted. Two problems with this technique are: the difficulty controlling bleeding from the multiple bony foramina in the floor and; the difficulty with exposing these foramina due to the resistance of bulky tumor above. Pre-operative super selective catheterization and embolization of the ethmoidal arteries is not without significant and prohibitive risk of blindness. A frontalethmoidal approach to the arteries requires 2 separate skin incisions. Sub periosteal, sub periorbital dissection and division of these arteries via the bicoronal skin incision is an alternative.

After turning a bicoronal scalp flap, sub periosteal dissection is carried out down over the orbital rims and nasion. The orbital surgeon then dissects in a sub periorbital plane along the medial wall of orbit using the anterior crest of the nasolacrimal fossa and frontoethmoidal suture as landmarks. Both foramina lie above the frontoethmoidal suture. The anterior ethmoid artery is found approximately $24 \mathrm{~mm}$. from the nasolacrimal crest where the artery can be clipped and divided. The posterior ethmoid artery, at $36 \mathrm{~mm}$. from the crest, and only $6 \mathrm{~mm}$. from the medial end of the optic canal, must be coagulated with bipolar forceps with the aid of operating loupes and headlight. After the arteries on both sides have been divided the craniotomy proceeds as planned.

This technique is simple, adds 20 minutes to the case at the outset, saving many more thereafter. An illustrative case and the relevant anatomy are presented.

\section{P92.}

Insertion of a Catheter - Ommaya Reservoir Construct into Tumor Cysts Using a Standard CRW Stereotactic Frame: Technical Note

M.W. McDermott, P.H. Gutin and M.S.B. Edwards (San Francisco, California)

The insertion of a catheter into deep seated tumor cysts for drainage or drug delivery can be accomplished easily using stereotactic methods. Connecting the catheter to an Ommaya reservoir dome, while maintaining the catheter position, is difficult. Using the standard CRW steriotactic frame without modification a technique has been developed to place a catheter Ommaya reservoir construct to a target point easily with one pass. The CRW base ring is attached to the skull in the usual manner and a stereotactic CT or MRI scan obtained. The distance between the outer table of the skull and the center of the tumor cyst is measured directly from the stereotactic coronal MRI images, preoperative MRI films or CT coronal reconstructions. A target in the center of the cyst is selected and then 
AP, LAT, VERT coordinates derived in the usual fashion. In the operating room the catheter - Ommaya reservoir construct is assembled to measured distance from the outer table of the skull to the center of the tumor cyst. A 25 gauge stainless steel wire is inserted through the dome of the Ommaya reservoir to the catheter tip. A burr hole is made, the dura opened and the CRW arc - ring system placed on the patient base ring after confirming the depth to target on the phantom base. A trajectory through a cerebrotomy is confirmed with a blunt stylet and then the drill guide block removed. The proximal end of the wire from the Ommaya is inserted back through a needle guide in the drill guide block. This unit (catheter - Ommaya construct, wire, drill guide block) is then delivered through the block collar on the CRW arc - ring system, passing the catheter tip into the cerebrotomy and moving the whole unit downward until the drill guide block abuts the collar. Then the catheter - Ommaya construct is passed to the target using the 25 gauge wire until the flange on the Ommaya stops at the outer table of skull, the wire removed and the Ommaya sutured in place. Photographs detailing the steps of the procedure and illustrative cases will be presented.

P93.

The Relationship Between Gender and Malignancy in Primary Brain Tumours

P.J. Muller, W. Tucker, R. Moulton, M. Cusimano and J. BILbAo (Toronto, Ontario)

It has long been recognized that primary malignant brain tumours have a male proponderence and that benign meningiomas are more common in the female population. We examined the relationship between gender and pathology in a series of gliomas and meningiomas diagnosed at St. Michael's Hospital from 1980 to 1991 .

Of 871 astrocytic tumours with a mean age of $55 \pm 12$ years 180 were low grade astrocytoma (grade 2), 328 were malignant astrocytomas (grade 3 ) and 363 were glioblastoma multiforme (grade 4). The male/female ratios were 1.25, 1.39 and 1.56, respectively ( $\mathrm{p}<0.005)$.

Of 376 meningiomas with a mean age of $58 \pm 14$ years 23 were histological malignant or atypical. The male/female ratio was 1.091 in comparison to 0.423 for the benign meningiomas $(\mathrm{p}<0.05)$.

There does appear to be a relationship between the degree of histologic malignancy and gender.

P94. The Use of Photodynamic Therapy in the Treatment of
Recurrent Single Cerebral Metastatic Disease

\section{P.J. MULLER and B.C. WILSON (Toronto, Ontario)}

Ten patients with metastatic cerebral tumours were treated with PDT. There were 5 males and 5 females; the mean age was $57 \pm 11$. The median Karnofsky score was $72 \pm 15$. There were 5 primary lung cancers, 2 breast, 1 colon and in 2 patients the primary was unknown. Photofrin $2 \mathrm{mg} / \mathrm{kg}$ i.v. was administered 12-24 hours prior to surgery. All had the tumour resected at craniotomy. The tumour resection was grossly complete in all but one patient leaving a cavity the walls of which consisted of edematous white matter. Intra-operative cavitary photo-illumination was carried out. The mean \pm sem tumour cavity volume was $15 \pm 3 \mathrm{~cm}^{2}$. The mean \pm sem light energy administered was $1780 \mathrm{~J} \pm 560$ and the mean energy density was $60 \pm 16 \mathrm{~J} / \mathrm{cm}^{2}$.

Histological assessment of the resection cavity which consisted of white matter grossly devoid of tumour revealed residual islets of tumour within the neuropil.

The median survival was 26 weeks post PDT with a range of 6 to 134 weeks. One patient is alive at 31 weeks post PDT and 3 lived longer than 1 year post PDT. Both patients who lived less than 30 weeks post PDT had advanced systemic disease. There were no postoperative deaths. One patient displayed worsening of their hemiparesis. There were no systemic reactions to Photofrin and no cutaneous toxicities.

Further study of PDT in the treatment of recurrent post radiation cerebral metastases is indicated.

P95.

Spinal Epidural Lipomatosis in a Patient with Prior Craniopharyngioma Resection

\section{VeILleux and P. Bourgoin (Montreal, Quebec)}

Spinal epidural lipomatosis is characterized by excessive adipose tissue deposited circumferentially around the spinal cord in the epidural space. It is most often associated with administration of exogenous glucocorticoids and localized in the thoracic region. A 23-year-old man with a history of subtotal craniopharyngioma resection at age 10 and secondary panhypopituitarism on thyroxin, testosterone and glucocorticoid replacement, complained in February 1993 of progressive lumbar and buttock pain radiating to the posterior aspect of the thighs and calves. There was no associated sensory symptom nor sphincter dysfunction. Neurological examination revealed a marked obesity but no signs of lumbosacral radiculopathy or cord compression. The pain progressively worsened to the point he could no longer get up from a chair and sleep on his back. A CT of the lumbar and cervical spine and an EMG of the legs were normal. However, MRI of the thoracic spine revealed severe posterior thoracic epidural lipomatosis. He slowly improved on medical treatment including weight and steroid reduction. We suggest patients with spinal epidural lipomatosis but without signs of cord compression should be first treated medically.

\section{II-4. SPINE}

\section{P96.}

\section{Multidisciplinary Development of a Teaching Booklet for complex Thoracolumbar Spinal Surgery}

M. Fehlings, V. Edmonds, F. Machale, H. Morris, T. EIMANTAS, M. MITKIEWICZ and S. RoBINSON (Toronto, Ontario)

Patient education is an essential aspect of high quality care. Complex spinal surgery has in recent years become an integral part of neurosurgery. To facilitate education of patients undergoing major thoracolumbar spinal surgery, a multidisciplinary 
team including occupational and physical therapy, neurosurgery, nursing and medical art was assembled to develop a teaching booklet. Interviews with previous spinal surgery patients were conducted to ensure that appropriate issues were addressed. A comprehensive, readable, illustrated booklet has been developed which reviews the preoperative, perioperative and postoperative patient-related issues required to provide high quality care. The presentation will demonstrate the format and layout of the booklet as well as pertinent content information. This booklet will be made available to other centres.

Supported by a grant from Codman.

\section{P97.}

\section{The Effect of Methylprednisone in Patients with Spinal Cord Injury}

S. LAL, M.I. KHAN and H. EL-AZZOUNI (Saskatoon, Saskatchewan)

In July 1990 steroid therapy, with methylprednisone, became standard therapy in patients with spinal cord injury. In this study, we analyzed/compared patients that did not received steroid therapy (July 1987 - July 1990) after spinal cord injury to those patients receiving a loading dose of methylprednisone, followed by 23 hours of intravenous therapy within 6 hours of spinal cord injury (July 1990 - July 1993). Patients with complete, partial and cauda equina injuries were examined. Over 100 patients presented the above injuries. Using chart review, interviews, and assessments from physiotherapy, functional improvement was assessed. Our study demonstrated that there was no improvement in complete cord injuries with the introduction of steroid therapy. In patients with partial cord injury there was significant functional improvement (p. 05). Details will be presented.

\section{P98.}

\section{Non-invasive Assessment of Spinal Function by the Spinoscope}

\section{A.E. SHAPTER and F.B. MAROuN (St. John's, Newfoundland)}

The principle that the skin over the spinous processes moves in the sagittal plane (X-axis) and the frontal plane (Z-axis) in a very distinct and consistent relationship to the underlying spinous process has been established. The Spinoscope System tracks the motion of infra red LED markers, taped to the skin overlying the spinous processes, with an opto-electronic motion analysis system at 180 images per second from each LED (Light Emitting Diode).

Skin surface EMG electrodes over the Multifudus provide data which is incorporated with the above data.

Basic spine co-ordination can be evaluated from this geometric and muscular information and compared with normals.

A normal or truly injured spine will, with repeated movements, produce similar results. The malingerer will not be consistent.

The spinoscope will provide such information as exact range of motion, spine and pelvic contributions, change in angle of lordosis seen in disc herniations and instability, intersegmental mobility, exact Schober test, muscle relaxation phenomenon, maximum lifting capacity, lateral bending sign in disc herniations, etc.

\section{P99.}

\section{Instability of the Lumbar Spine Measured by the Spinoscope}

\section{A.E. Shapter and F.B. Maroun (St. John's Newfoundland)}

Intersegmental instability of the lumbar spine occurs when there is loss of disc support, interspinous and/or supraspinous ligament damage, facet capsule laxity due to tears or facet arthrosis, in various degrees of severity.

The spinoscope measures the separation distances between each spinous process in flexion and extension sequences, and in lateral bending sequences. It then computes the percentage differences very quickly.

Twenty adults with clinical evidence of lumbar spine instability where examined on the spinoscope pre- and post-operatively. The operative findings were carefully recorded. All were fused posterolaterally.

Taking $60 \%$ separation in flexion, and $10 \%$ each side as the upper limits of normal, the spinoscope findings were shown to correlate well with the operative findings, even in detecting nonunion of the fusion, as shown by the excess of movement persisting.

P100.

Combined Microneurosurgical and Video Thoracoscopic Removal of Dumbbell Thoracic Spine Tumors

J.M. Findlay, E. Vallieres and R.E. Fraser (Edmonton, Alberta)

Dumbbell throacic spinal tumors, which extend from the spinal canal through the intervertebral foramen into the thoracic cavity, are usually benign neoplasms originating from the nerve sheath, and until now their resection has usually required laminectomy followed by thoracotomy. Over a one year period a combined posterior microsurgical and anterior thoracoscopic tumor removal was attempted in four patients. Two patients presented with myelopathy and two with posterior mediastinal masses on chest $\mathrm{x}$-ray. All had large intraspinal and intrathoracic tumor components, and the pathology was schwannoma in 3 and benign mesenchymoma in one. The first stage of the procedure consisted of laminectomy and foraminotomy for microsurgical excision of the intraspinal and intraforaminal tumor components, along with circumferential cauterization and mobilization of the tumor capsule as it entered the thoracic cavity. This incision was then closed and following reintubation with a doublelumen endotracheal tube the patients were positioned laterally. Four-port thoracoscopy permitted successful intrathoracic tumor removal in 3 patients, but one procedure had to be converted to a thoracotomy because of what appeared to be uncontrollable hemorrhage from a divided intercostal artery. In the three patients with successful thoracoscopic resections postoperative discomfort was significantly less and recovery more rapid. All patients made a good recovery. Because of reduced morbidity, 
we believe that video assisted thoracoscopic surgery will become the preferred method of removing the intrathoracic part of benign thoracic spine dumbbell tumors. Suitable patients and the technique will be described in detail.

\section{P101.}

Spinal Injuries at Royal University Hospital, Saskatoon, Saskatchewan - A Five Year Study

S. LAL, H. El-Azzouni and M.I. HaN (Saskatoon, Saskatchewan)

This is a 5 year (1987-1992) retrospective study of spinal injuries referred to the neurosurgical/orthopedic services at Royal University Hospital, Saskatoon, Saskatchewan, Canada (population base of approximately 500,000). A total of 468 patients (65\% males, 35\% females) were admitted. $52,20,28 \%$ of patients suffered from cervical, thoracic, or lumbar injuries respectively. The majority of injuries occurred in the summer months $(36 \%) .82 \%$ patients were Caucasian, $16 \%$ Native Indian, and $2 \%$ were of other origins. $50 \%$ of patients were between the ages of 11 and $30.60 \%$ of spinal injuries were incurred in motor vehicle accidents (MVAs) with alcohol present in $33 \%$. The majority of natives $(93 \%)$ were involved in MVAs. Seatbelts were not worn in most patients and in these patients a large proportion had taken alcohol. In only $2 \%$, deaths occurred. The average stay in hospital was 26 days and most received conservative management. The epidemiological data gathered will hopefully guide preventative measures in the future and details of the study will be presented.

\section{P102.}

Delayed Myelopathy after Trivial Neck Injury in a Patient with a Cervical Neuroenteric Cyst

\section{R. Midha, B. Gray, L. Becker and J. Drake (Toronto, Ontario)}

Neuroenteric cysts are rare spinal lesions of congenital origin. We report the unusual presentation of a patient who developed a progressive myelopathy after minor neck trauma and proved to have this on the basis of a neuroenteric cyst. A 14-year-old previously well male slipped at a skating rink, striking the back of his head on the ice and sustained a mild flexion injury to his neck. Over the next three days, a slowly worsening right hemiparesis developed, associated with urinary dysfunction. Motor examination disclosed right upper and lower extremity weakness, with decreased power (MRC 3-4/5), more prominent distally. Tone and reflexes were increased in the right side, along with sustained right ankle clonus and bilateral Babinski signs. Decreased sensation was confined to the C5 dermatome. Plain cervical spine films were normal. MRI demonstrated a large intradural, extramedullary cystic lesion in the spinal canal extending from $\mathrm{Cl}$ to $\mathrm{C} 4$, causing marked compression and displacement of the cord. The lesion was of a similar intensity to CSF and failed to enhance with gadolinium. At operation, after a C2-4 cervical laminectomy, a taut intradural cyst, containing clear fluid without any trace of blood was aspirated, excised and widely fenastrated to decompress the spinal cord. The patient experienced progressive recovery in his right hemiparesis and resolution of his urinary symptoms. Pathologic exam of the cyst showed a ciliated columnar epithelium which stained positively for mucin, confirming its enterogenous origin. Neuroenteric cysts usually present insidiously with a long history of local spinal or radicular pain and/or myelopathy. The present case is unique in its short history and apparent precipitation of neurologic deterioration by trauma. Neuroenteric cysts, in general, will be reviewed as will the possible pathogenesis of the present case in relationship to the minor cervical spine injury.

P103.

Prognostic Factors in Cervical Myelopathy Surgery, Saskatoon, Saskatchewan (10 Years Retrospective Study)

\author{
R. MaZagri, M. Khan and G. Goplen (Saskatoon, \\ Saskatchewan)
}

In this retrospective study we analyzed the outcome of surgical treatment of 25 patients with cervical myelopathy secondary to cervical spondylosis from 1982 to 1992 . Each patient presented with the classical signs and symptoms of cervical myelopathy. All patients underwent decompressive cervical laminectomy.

The age range is from 45 to 84 with a mean of 72 years. Each patient presented with sensory and motor long tract signs. There were 16 males and 9 females. There was evidence of multilevel involvement by myelogram and/or CT scan investigations. Period of follow-up ranged from 6 months to 6 years. In this study, factors influencing the outcome of surgery such as age, sex, occupation, history of trauma, duration of symptoms, severity of the disease at time of presentation, and the severity of the disease on radiological investigation were studied.

Details of the study will be discussed.

P104.

\section{Lumbar Spondylosis Causing Intermittent Neurogenic Claudication of the Cauda Equina}

M.P.B. Kilburn, M. Khan and G. Goplen (Saskatoon, Saskatchewan)

A retrospective study of 58 patients admitted to Royal University Hospital with the clinical syndrome of intermittent neurogenic claudication between 1982-1992 was analyzed. We included only patients who presented with symptoms made worse by walking or activity and relieved by rest who demonstrated no evidence of vascular insufficiency by clinical and radiological examination. All patients in the study showed radiological evidence of lumbar spondylosis by myelogram with or without CT scanning. The study population included 29 men and 29 women between the ages of $26-83$ with an average age of 64.6 years. Occupationally most patients were involved in farming. The average duration of symptoms was 4.86 years. Twentynine patients presented with bilateral signs of cauda equina root dysfunction. Average follow-up was 15 months. Post operatively at follow-up $35 \%$ of patients had no symptoms, while $36 \%$ had minor symptoms and $18 \%$ had symptoms which limited them 
but were improved overall. Only $7 \%$ of patients had no change in their symptoms and only $4 \%$ were felt to have deteriorated following surgery. A detailed analysis will be presented.

\section{P105.}

\section{Spinal Cord Meningioma}

M.P.B. Kilburn, R.W. Griebel and G. Goplen (Saskatoon, Saskatchewan)

A retrospective review of 37 patients with spinal meningioma was performed with a view to analyzing long term recovery and recurrence rates. Age, sex, tumor location and clinical presentation are similar to those reported by others. Before surgery $78 \%$ were included in grades 0 , I and II and $22 \%$ in grades III and IV in terms of their motor function and ability to ambulate. Surgical treatment resulted in only one patient having increased deficit. No change occurred in 3 patients and in the remaining 33 patients their motor function had improved to full recovery. This improvement in function persisted over an average of 7.6 years. Histologically all of the tumors fell into the usual groupings with two patients showing atypical features. Overall there were no recurrences. Factors such as tumor location relative to the spinal cord, use of intraoperative microscope and presenting signs and symptoms did not statistically affect the eventual outcomes for these patients.

A detailed analysis will be presented.

\section{P106.}

\section{C1-C2 Transarticular Screw Fixation in the Treatment of C1-C2 Instability}

T.J. CoYne, M.G. Fehlings and R. Martin (Toronto, Ontario; Syracuse, New York)

$\mathrm{C} 1-\mathrm{C} 2$ instability has traditionally been treated by $\mathrm{C} 1-\mathrm{C} 2$ posterior wiring and bone grafting. However, this technique has an incidence of non-union which may exceed 10\%. C1-C2 transarticular screw fixation has recently developed as a technique of providing increased strength of fixation when performing $\mathrm{Cl}-\mathrm{C} 2$ arthrodesis, while minimizing the need for post-operative bracing and avoiding the risk of neurological injury associated with the passage of sublaminar wires. We present a retrospective review of 12 patients with $\mathrm{C} 1-\mathrm{C} 2$ instability treated by $\mathrm{Cl}-\mathrm{C} 2$ transarticular screw fixation. Eight patients underwent this procedure as primary treatment, and 4 after a failed Gallie fusion. Five patients had a cruciate ligament rupture, 4 had an odontoid process fracture, 1 had os odonteum, and 1 had rheumatoid instability. There was no surgical morbidity or mortality, and at a mean follow-up of $12.1 \pm 3$ months (range 8-14 months), all patients had achieved solid fusion, and all neurological symptoms referable to the instability had resolved. In addition to presenting these patients, the surgical technique employed is illustrated. $\mathrm{Cl}-\mathrm{C} 2$ transarticular screw fixation has been shown to be safe and effective, and has a number of advantages when compared to traditional posterior wiring techniques. We recommend that this technique be considered as a primary treatment of $\mathrm{C} 1-\mathrm{C} 2$ instability.
P107.

Management of Cervical Instability with Lateral Mass Plates: Early Results in a Prospective Series of 18 Patients

\section{FAYAZ and M.G. Fehlings (Toronto, Ontario)}

Posterior cervical plates are being increasingly used to manage cervical spinal instability. To date, however, reports have been limited to a retrospective review. We have prospectively assessed our early experience in 18 patients: 11 males and 7 females, age range 17-77, from July 1992 to January 1994. Instability was due to trauma $(n=15)$, tumor $(n=1)$, degenerative disease $(n=1)$, and amyloidosis $(n=1)$. Five of the 18 patients required an anterior fusion in addition to posterior cervical plating. Two hole plates were used in 9 patients to achieve single motion segment stabilization, with 3,4 , and 5 hole plates being utilized in 6,1 , and 2 patients respectively. As a novel technical modification, pedicle screws were inserted at $C 2(n=$ $2)$, and lateral mass screws at $\mathrm{Tl}(\mathrm{n}=1)$. All patients underwent autologous iliac crest bone grafting. Follow-up was complete in all but one patient, and ranged from 2-19 months. There was no mortality in our patient group. Peri-operative morbidity consisted of one superficial wound infection, a DVT, and pneumonia $(n=3)$. At the time of follow-up, all patients were noted to either maintain, or show improvement, in their ASIA impairment scale. All patients achieved stability (100\%), and no patient has yet required a revision. Fifteen patients have shown solid bony fusion at greater than 3 months of follow-up. In $88 \%$ of patients, excellent correction of kyphotic deformity was obtained. The screw pullout rate was $1 / 88$, or $1.1 \%$. Our early results clearly attest to the technical ease, safety, efficacy, and cost-effectiveness of this instrumentation system in the management of destabilizing injuries to the cervical spinal column.

\section{P108.}

\section{Central "T" Griseotomy: A Treatment of Choice in Selected} Cases of Spasticity

\section{R. Moumdian and P. DuQuette (Montreal, Quebec)}

Operative procedure aiming at treating spasticity are numerous including central " $T$ " griseotomy (CTG). CTG produces flaccidity without any muscular atrophy and causes segmental analgesia only. All long tracts are preserved and propiospinal fibers are disrupted; this eliminates all afferent influx from inferior and superior spinal segments usually responsible for recurrent spasticity.

The surgical indication concerns patients with severe spasticity of the lower limbs and unable to walk with or without pain.

Four patients with severe multiple sclerosis were treated with initial $100 \%$ (4/4) success with a follow up of 22 to 36 months. One patient recurred 16 months after without pain.

CTG relieved both spasticity and pain; it facilitated patient hygiene. One patient died 8 weeks postop from pulmonary embolism. She had had several episodes of lower limb deep vein thrombosis.

We conclude that CTG should be considered as a treatment alternative in some patients with severe lower limb spasticity. 
P109.

Alterations in the Normal Pattern of Cervical Spinal Cord Motion as Measured by MRI in Selected Pathologic States

D.J. Mikulis (Toronto, Ontario)

\section{Purpose}

To demonstrate how the pattern of normal spinal cord motion is altered by abnormalities of the neural axis.

Material and Methods

Two dimensional phase subtracted MR imaging sequences were performed in normal volunteers and in patients with abnormalities of the neural axis to assess movement of the spinal cord in relation to the cardiac cycle. Following cardiac systole, 12 22 heart phases at $25-30 \mathrm{msec}$ intervals were collected. Velocity information was calculated by performing phase subtractions on regions of interest within the spinal cord. Data acquisition was performed in the axial or sagittal plane at the level of C3 for all measurements. Duration of the imaging sequences for the study of cord motion was 2 - 4 minutes.

Results

The cervical spinal cord always moved in a caudal direction during the initial phase of the cardiac cycle reaching a maximum velocity of $6-12 \mathrm{~mm} / \mathrm{sec}$ between 60 and $130 \mathrm{msec}$ after systole followed by an alternating cranial and caudal motion of progressively decreasing velocity. Patients with spinal cord compression, tethered spinal cord syndrome, and intracranial high flow vascular malformations showed either reduced or increased spinal cord velocities respectively.

\section{Conclusion}

The spinal cord clearly moves in an oscillatory pattern following cardiac systole in normal volunteers. Reduced motion occurs in patients with disorders that compress or tether the spinal cord. Increased motion can be seen in patients with intracranial high flow vascular malformations. The ability to assess and grade cord motion may provide clinically useful information in establishing the degree of spinal cord compression or tethering.

\section{P110.}

\section{Spinal Surgery in the Former Soviet Union}

E.G. Duncan, A. North and R. Hansebout (Mississauga; Windsor; Hamilton, Ontario)

In October, 1993, the authors were among a group of ten neurosurgeons which visited spinal units in three cities (St. Petersburgh, Moscow and Minsk) in the former Soviet Union. We visited seven acute care hospitals and one rehabilitation unit, all national centres, where we met medical staff and toured the wards. Several symposia were held with both host and guest presentations

In general, patients with acute spinal injuries, with or without neurologic deficit, are initially managed in Institutes of Traumatology staffed by orthopaedic surgeons. These units also treat patients with chronic spinal instability including scoliosis. Neurosurgical Institutes primarily manage non-traumatic spinal abnormalities and some patients with persisting traumatic neurologic deficits.
Overall, the knowledge and skills of the surgeons were impressive. However, chronic lack of funding was obvious. Hospitals were in poor repair, wards were crowded and access to $\mathrm{CT}$ and MR scanning limited. There were no electronic monitoring systems in the one ICU that we visited.

Spinal instrumentation is used. The systems most often seen were a unilateral strut system and Harrington rods. However, a spinous process external fixateur, a variation on the Halifax clamp and a prototype pedical screw system were also seen.

One significant difference in treatment concerned the late management of spinal cord injury. Our hosts believe in late recovery of function in complete injuries and reoperate even after several years. Procedures include lysis of adhesions at the injury site. Peripheral nerve grafts, as cable grafts for cauda equina injury or as transposition grafts into the spinal cord were also described.

The professional component of our tour alone made the visit worthwhile. However, we also saw many cultural marvels, including the glories of St. Petersburgh, the czarist capital of Russia. Our arrival on the eve of the uprising around the "White House" ensured that this visit would be particularly memorable.

\section{P111.}

\section{Spinal Cord Electrical Stimulation for the Treatment of Refractory Angina Pectoris}

L. Jacques, N. Martinez, R.M. Gagnon, D. Savard and D. RENÉ (Montreal Quebec)

Twelve patients with refracting angina pectoris previously treated with angioplasty, coronary by pass under optimal medical therapy were treated with spinal cord electrical stimulation (SCES) at the C7 to D2 level since 1988.

Six patients had a significant improvement of symptoms confirmed by thallium persantin test, reduced incidence of chest pain and decreased use of nitroglycerine.

Three patients required removal of electrodes (heart transplantation, inadequate comprehension and used of method, foreign body reaction). These patients did however benefit from the effect of SCES for some times. Three patients died.

The SCES may improve the quality of life by reducing the incidence of chest pain in patients previously uncontrolled on maximal medical and surgical therapy.

\section{P112.}

Factors Affecting Discharge of Patients After a Lumbar Discectomy

\section{E.A. BADEJo (Kearney, Nebraska)}

This is a review over a 5 month period of patients operated on for lumbar discectomy. In the last 5 months there were 29 patients who underwent 32 procedures. The average stay was 2.4 days. The range was 1 to 6 days.

The longest stay was in patients who were more than 70 years old. The shortest stay was in patients who were between 21 and 30 years old. 
The most significant factor that affected length of stay was age. The average stay for patients 70 years and above was 4.25 days. In patients who were 60 and below, the average length of stay was 2.1 days, and 3.1 days in those older than 60 years.

The use of the microscope did not have any significant affect on the length of stay.

The major factors in prolonged stay in the hospital following lumbar discectomy are the surgeon's reluctance for early discharge and age of patient.

There were 3 complications in this series. All 3 were recurrent disc herniations. There was no infection.

\section{P113.}

\section{Early Discharge Following Anterior Cervical Discectomy and Fusion}

\section{E.A. BADEJO (Kearney, Nebraska)}

This is a review of the length of hospitalization in patients who had anterior cervical discectomy and fusion for cervical disc herniation over a 5 month period of time.

There were 12 patients who underwent 13 procedures. 2 patients had Smith-Robertson procedure with cadaver bone graft used. All the other patients had Cloward Procedure.

The average length of stay in the hospital postoperatively was 2 to 4 days. The shortest stay was 1 day, and the longest stay was 4 days. 8 of the 13 cases were discharged in 2 days or earlier.

The major factors for prolonged stay were age and pain from graft site.

The only complication was extrusion of the bone grafts in 1 case.

Conclusion: Most patients can be safely discharged home 2 days or less following anterior cervical discectomy and fusion.

\section{II-5. GENERAL NEUROSURGERY}

\section{P114.}

The Relationship Between Glutamate Release in Cerebrospinal Fluid and Intracranial Physiological Variables in Head Injury

\section{S.J. Konasiewicz, A.J. BaKer and R.J. Moulton (Toronto, Ontario)}

To explore the relationship between excitatory amino acid (EAA) release and cerebral physiological variables in severe head injury (GCS $\leq 8$ ), glutamate levels in cerebrospinal fluid (CsF) were compared to cerebral blood flow (CBF), the cerebral arteriovenous oxygen difference (AVDO2), the intracranial pressure (ICP), the cerebral perfusion pressure (CPP), and the rate of cerebral oxidative metabolism (CMRO2).

Upon admission to the ICU, patients were treated with a standard protocol. Csf was collected every 12 hours and frozen. Samples were analyzed by high-performance liquid chromatography for detection of glutamate. Concurrent measurements of $\mathrm{CBF}$ (using the $\mathrm{N} 2 \mathrm{O}$ clearance technique), AVDO2, CMRO2, ICP and CPP were made.

Data from 15 patients (age $51 \pm 3.45$ years; sampling duration $3.95 \pm 0.57$ days; means $\pm \mathrm{SEM}$ ) were analyzed. Glutamate measurements above the normal range ( $>20$ micromolar, $\mathrm{n}=$ 36) were compared to those that were in the normal range $(\leq 20$ micromolar, $n=40$ ) and were found to be associated with a highly significant $(\mathrm{p}<.0001)$ lower CBF $(34.42 \pm 1.38$ vs. $45.96 \pm 2.46 \mathrm{cc} / 100 \mathrm{~g} / \mathrm{min}$ ) higher AVDO2 (5.20 \pm .27 vs. $3.67 \pm$ .23 volumes \%) and higher ICP (18.64 \pm 1.31 vs. $10.53 \pm 1.17$ $\mathrm{mm} \mathrm{Hg}$ ). No significant differences were found between CPP and CMRO2 levels for the normal and abnormal Csf glutamate levels $(p=.31$ and $p=.39$ respectively, student $t$-test).

From a clinical point of view, measures that aggravate ischemia should be avoided to minimize EAA release and to lessen subsequent neurotoxicity that may evolve after severe head injury. Furthermore, excess glutamate release may not be related to levels of CPP.

\section{P115.}

\section{TCD in Acute Head Trauma}

F.Y. Hsieh, A.V. Alexandrov, M.L. SchwartZ and J.W. Norris (Toronto, Ontario)

Transcranial doppler ultrasonography (TCD) may be used to monitor patients with raised intracranial pressure and could be a valuable adjunct in acute head trauma.

We performed TCD evaluation daily for the first five days, and weekly for two weeks in all patients admitted to our centre within four hours of brain injury, and correlated these data with clinical findings (Glasgow Coma and Outcome scale), brain CT and intracranial pressure (Richmond Screw).

Of a total of 21 patients, seven were excluded for technical reasons. In the remaining 14 there were three patterns of blood flow velocities (BFVs). (1) Normal, 6 cases. (2) Parallel increases of mean BFVs in both cerebral hemispheres, 1 case. (3) Discrepant changes in mean BFVs, 7 cases.

The major cause of increased BFV's appears to be compression, either by ipsilateral hemispheric pathology or by contra-lateral hemispheric shift.

These inital data suggest that TCD monitoring may be a useful adjunct in the acute management of severe head trauma.

\section{P116.}

\section{Altération de la Phosphorylation de la Thiamine dans les} Maladies Neurodégénératives

M. D'Amour L., J. Lavole, V.L. Raghavendra Rao and R.F. ButTERWORTh (Montréal, Québec)

Plusieurs évidences suggèrent que la phosphorylation de la thiamine est altérée dans les maladies neurodégénératives comme la maladie d'Alzheimer (MA) et la sclérose latérale amyotrophique (SLA). Dans le but d'évaluer cette possibilité, les esters de thiamine et leurs enzymes ont été mesurés dans le tissu cérébral autopsique de patients avec la MA et de contrôles appariés. La thiamine et ses esters mono, di, et triphosphate ont été mesurés dans le LCR par une technique de CLHP. Les enzymes thiamine mono et di-phosphatases ont été mesurés dans le tissu cérébral par une technique spectrophotométrique. L'activité de la thiamine diphosphatase (TDPase) est réduite dans le cortex frontal (diminution de $28 \%$ ), $\mathrm{p}<0,05$ ) et le cortex 
temporal (diminution de 62\%), $\mathrm{p}<0,01$ ) des patients avec la MA. L'activité de la thiamine monophosphatase (TMPase) est aussi réduite (diminution de 65\%, $\mathrm{p}<0,01$ ). Des résultats préliminaires suggèrent que les concentrations de la thiamine monophosphate et de la thiamine libre sont élevées de 2 à 3 fois dans le LCR des patients avec une SLA. L'altération des mécanismes de phosphorylation-déphosphorylation peut donc être impliquée dans les mécanismes de la mort neuronale dans les maladies neurodégénératives.

(Subventionné par le CRMC.)

\section{P117.}

Pre-Operative CT and MRI Findings in Patients Undergoing Functional Hemispherectomy for Intractable Seizures

\section{Dureza, J.-G. Villemure and D. Tampieri (Montreal, Quebec)}

Since its introduction in 1974 at the MNI the functional hemispherectomy has been used successfully to treat patients with intractable seizures due to a number of etiologies. This study was aimed at identifying the pre-operative CT and MRI characteristics of patients undergoing this procedure.

From 1982 to 1990, 27 patients underwent a functional hemispherectomy in the manner of Rasmussen for intractable seizures. These patients were diagnosed pre-operatively with one of the following: encephalitis, perinatal ischemic event, post-traumatic seizures, Sturge-Weber, megalencephaly, or dysplasia. Pre-operatively 13 patients had a CT scan, while 14 had MRI studies. Of these patients 9 had both a CT scan and MRI. In all patients the pathological hemisphere was clearly seen by both CT scan and MRI. The CT scan was quite useful in localizing the defective side through gross abnormalities. Serial CT scans were useful in following the progression of an encephalitic process. CT was also more likely to pick up abnormal calcifications. The MRI was better able to define the exact nature of the hypodensities seen on CT scans. The MRI showed more anatomical detail such as cerebral peduncular atrophy as well as defining the extent of previous surgery.

From this study it is evident that one should obtain both a CT scan and MRI prior to performing a functional hemispherectomy. With the addition of MRI one can better define the subgroup within this population of patients who can most benefit from a functional hemispherectomy.

\section{P118.}

Single Vessel Lesion in Transient Ischemic Attacks (TIAs): Angiographic Study

M. Assaf, K. Gaines, A. Elotti and J. Acker (Memphis, Tennessee)

We retrospectively analyzed the findings from 4-vessels angiography in 312 pts (whites: 141 males, 130 females; blacks: $20 \mathrm{M}, 21 \mathrm{~F}$ ) with TIAs based on ICD codes in an effort to determine the frequency of $>40 \%$ single vessel stenosis (SVS) in this population. The age, sex and race differences will be analyzed.
Abnormal angiogram was demonstrated in $57 \%\{59 \%$ of whites, $44 \%$ of blacks\} [ $60 \%$ of males, $54 \%$ of females]. The frequency of abnormal angiograms were $50 \%$ high in pts $>60$ years of age. Angiogram were found abnormal in the posterior circulation $14 \%$, anterior circulation $48 \%$ and $33 \%$ in both. 60 patients $(34 \%)\{60 \% \mathrm{M}, 40 \% \mathrm{~F}\}$ were found to have SVS, $77 \%$ in the carotid distribution and $23 \%$ in the posterior circulation. $55 \%$ in the proximal internal carotid artery, $17 \%$ in the vertebral artery, $10 \%$ in the middle cerebral artery, $1-3 \%$ for each of the common carotid, anterior carotid, internal carotid at the siphon, posterior cerebral and basilar arteries.

We conclude that abnormal angiogram and SVS in TIAs is more common in white, male $>60$ yrs of age pt with extracranial carotid artery disease. More pts are required to determine the need for 4-vessels angiography in specific groups of pts with TIAs.

P119.

\section{Cirsoid Aneurysms of the Scalp}

R. Moumdian, A. Chidiac, M. Bojanowski, D. Roy and R.-P. Delorme (Montreal, Quebec)

Cirsoid aneurysms (angiomas) of the scalp are rare lesions. They can result from a traumatic lesion to the scalp; more often they are congenital arteriovenous malformations or fistulae located in the galea aponeurotica. Patients usually present for cosmetic reasons, pulsatile headaches, bruit and even profuse bleeding.

Two such cases were treated recently in our medical centre. One underwent embolization, the second both embolization and surgery. Total obliteration was obtained in both. Very often the skin overlying the angiomas has to be removed together with the vascular malformation especially in embolized cases to prevent necrosis and secondary dehescene or infection.

Although some giant angiomas are not amenable to treatment, many may be treated successfully with embolization and/or surgical resection as well as reconstructive surgery.

\section{P120.}

The Syndrome of Superficial Cerebrospinal Siderosis and Treatment by Spinal Fluid Shunting

T.G. Staunton, R. Duke and J. GiRvin (Hamilton; London, Ontario)

Until recently Superficial Cerebrospinal Siderosis (S.C.S.) has been almost exclusively a post mortem diagnosis, recognized in cases of known intracerebral bleeding (e.g., post-hemispherectomy or subarachnoid haemorrhage). However, Magnetic Resonance Imaging can readily reveal superficial deposition of Iron over the cerebral convexity and the spinal cord. This makes clinical suspicion and diagnosis of S.C.S. Syndrome, typified by progressive sensorineural deafness, myelopathy with ataxia and eventual cognitive compromise, imperative. In particular, such patients without history of subarachnoid haemorrhage or trauma must be aggressively investigated for a source of chronic subarachnoid bleeding. We 
described two patients, one with a history of remote excision of a low grade brain stem neoplasm, and the other with no identifiable source of subarachnoid haemorrhage despite intensive investigation. Both have the typical SCS syndrome and had chronically grossly blood stained spinal fluid. Both have been treated by cerebrospinal fluid shunting procedures with remarkable clearing of the spinal fluid and apparent stabilization of their clinical status.

\section{P121.}

\section{Control of Parkinsonian Tremor with Chronic Thalamic Stimulation}

A.M. Lozano, R.R. Tasker, E. Sime, A.E. LAng and J.O. Dostrovsky (Toronto, Ontario)

During the surgery for Parkinson's disease neurosurgeons have found that a brief burst of electrical stimulation in the ventral intermediate nucleus of the thalamus can arrest tremor. This observation has spearheaded the use of chronic stimulation of the thalamus as an alternative to thalamotomy for the control of tremor. We report our experience with chronic thalamic stimulation for tremor in three patients with idiopathic Parkinson's disease. The thalamus was explored using microelectrodes. Neurons in the ventral caudal somatosensory nucleus and the ventral intermediate nucleus (VIM) were identified by their characteristic receptive fields. We identified "tremor cells" in VIM whose discharge closely followed tremor rhythmicity as recorded by EMG. The permanent stimulating electrode was positioned at the site of lowest threshold for microstimulation induced tremor arrest and connected to a subcutaneously implanted pulse generator. Turning on the stimulator produced cessation of tremor activity within one second in all patients. The most effective parameters for stimulation were 150-180 hertz, 100-200 millisecond pulse width, and 2-4 milliamps of current. Chronic thalamic stimulation may be effective in treating tremor and has the advantage over thalamotomy of being non destructive.

\section{II-6. NEUROLOGY - NEUROMUSCULAR}

\section{P122.}

Left Leg Monoparesis Due to Lumbosacral Plexus Avulsion Injury: A Case Report and Review of the Literature

\section{M.B. MARKS (Ottawa, Ontario)}

Lumbosacral plexus avulsion injuries are not common due to the marked stability provided by the pelvis. The majority of cases previously reported show involvement of one to three root levels. The case of a 29-year-old male with left lower extremity monoparesis following a motorcycle accident is presented. EMG findings were consistent with a L2-S2 nerve root avulsion injury. CT-Myelogram examination confirmed avulsion of the left L3-S2 roots inclusively. Radiologic features, the use of EMG examination in making the diagnosis and a review of previously documented cases of lumbosacral root avulsion injuries will be included.
P123.

Selective IgG1 and IgG3 Deficiency in Patients with Amyotrophic lateral Sclerosis

\section{B. Ostermeyer Shoaib and B.M. Patten (Houston, Texas)}

In order to get clues about T-cell independent versus T-cell dependent immune mechanism in ALS, we measured IgG subclasses in 25 ALS-patients: sixteen patients had deficiency of T-cell dependent expressed IgG1 or IgG3 or both with essentially normal levels of T-cell independent expressed IgG2 and IgG4. Ten of these patients had no prior treatment and five out of these 10 had normal total IgG. Six patients had some immunosuppressive treatment before measurements of subclasses were done and all of them had deficiency of total IgG.

Eight out of 14 patients who underwent a d-xylose breath test, had evidence of small bowel overgrowth, which was confirmed by cultures of duodenal aspirate.

IgG1 and IgG3 are T-cell dependent antibodies against protein antigens with close linkage on chromosome 14. The findings suggest a defect in the IgG subclass expression in ALS.

\section{P124.}

\section{Motor Evoked Potentials in Miller Fisher Syndrome: Further Evidence Against Proximal Demyelination}

\section{Z. NASREDDINE and F. GRAND'MaISON (Sherbrooke, Quebec)}

The Miller Fisher variant of Guillain-Barré syndrome has generated conflicting opinions regarding the axonal versus demyelinating nature of neural involvement. In a recent study, it was suggested that electrodiagnostic abnormalities were distinct from the usual features of Guillain-Barré syndrome and were characteristic of an axonal sensory neuropathy or neuronopathy. However, proximal motor nerve conduction slowing, which is difficult to detect on routine nerve conduction studies, could not be excluded. We therefore set out to further assess proximal motor conductions by measuring the latencies of cortically evoked motor potentials in a patient with this condition.

Motor evoked potentials were recorded over triceps, intrinsic hand muscles and tibialis anterior bilaterally following transcutaneous cortical magnetic stimulation 6 days and 22 days after the onset of symptoms. Latencies were normal over all muscles tested, even in the left triceps which showed mild weakness and a slightly neurogenic recruitment pattern on needle EMG. Routine motor nerve conduction studies including F-wave latencies were normal. Sensory nerve action potentials were either absent or small in amplitude.

Normal motor evoked potential latencies to limb muscles provide further evidence against proximal demyelination in the Miller Fisher variant of Guillain-Barré syndrome.

'Fross RD, Daube JR. Neuropathy in the Miller Fisher syndrome: clinical electrophysiological findings. Neurology 1987; 37: 1493-1498. 
P125.

\section{Juvenile Muscular Atrophy of Hirayama Type}

J.-P. Bouchard, M. Thibault, D. Brunet and J.P. Antel (Quebec; Montreal, Quebec)

Since 1959, Hirayama and other Japanese authors have described a peculiar motor neuron disease in more than 150 cases. It consists of rapid, self-limited, distal amyotrophy of one upper extremity in young males (15-25 years). Cases have also been recorded in India and Europe.

We describe the case of a 17-year-old French Canadian male who consulted in January 1990 for rapidly progressive amyotrphy of the left hand and forearm. There was no sensory deficit. On EMG examination, no fibrillation of fasciculation was recorded, the number of motor units was greatly reduced, and giant potentials were present in hand muscles. The brachio-radialis was typically spared. Anti-GM $\mathrm{GM}_{1}$ antibody titres were in the normal range. In the following months, there were overt fasciculations, weakness and atrophy of the left pectoral and arm muscles. Cervical MRI revealed atrophy of the spinal cord from C-5 to upper thoracic segments.

The patient has now been stable for the last 3 years, but still presents amyotrophy and moderate weakness of the left hand. There are only rare fasciculations and hyperreflexia, but no weakness in the left upper arm.

Conclusion: In young men, progressive unilateral amyotrophy of one hand may present a good prognosis.

\section{P126.}

\section{Traumatic Pudendal Neuropathy and the Dimple Sign}

\section{T.G. StaunTon (Oakville, Ontario)}

Blunt trauma to a peripheral nerve may result in little objective clinical deficit especially if the nerve has predominantly sensory function. Such is the case with the posterior cutaneous nerve of the thigh and also the pudendal nerve, which both exit posteriorly from the pelvis via the sciatic foramen passing around the sacrospinal ligament. Local compressive trauma of these adjacent nerves may be caused by blunt injury to that region just lateral to the coccyx, which may spare the even more lateral sciatic nerve. I describe the history and findings in 3 women who received blunt trauma to one buttock ( 2 in motor vehicle accidents and 1 in a fall at work) resulting in ipsilateral vaginal, perineal and posterior thigh hypesthesia with dysesthesia, only one of whom demonstrated abnormal nerve conduction/EMG findings. However, all 3 developed focal dimpling of the buttock as a result of traumatic fat necrosis. This "dimple sign" may be significant as objective clinical evidence which corroborates and correlates with symptomatology, aiding diagnosis. This may be important if liability is involved.
P127.

A Case of Infiltrative Squamous Cell Carcinoma of the Skin Presenting as Multiple Cranial Mononeuropathies

R. Ganesan, P. Matthews and R. McKelvey (Montreal, Quebec)

Squamous cell carcinomas are known to metastasize, often by local spread. Reports have previously described infiltrative cranial neuropathies, particularly of branches of the trigeminal nerve. We report here one case of sequential trigeminal and facial nerve involvement progressing over 8 months.

A 66-year-old man with a prior history of resections of basal and squamous cell carcinomas presented with persistent pain over the right nasal bridge. Subsequently, the pain spread to involve the forehead and maxillary areas. Eight months later, he developed a progressive right facial droop including loss of the frontalis creases. Investigations as an outpatient, including repeat cranial CTs and MRI, were negative despite the extensive fifth and seventh cranial nerve involvements. Further imaging in hospital, including CTs of the skull base with bone windows, failed to show evidence of erosive tumour. Repeat lumbar punctures were unremarkable apart from a mildly elevated protein. Finally, an infraorbital nerve biopsy confirmed extensive neural invasion by squamous cell carcinoma.

This report illustrates an unusual case of infiltrative squamous cell carcinoma presenting as multiple cranial mononeuropathies without extensive bony erosion.

\section{P128.}

A Case of a Biopsy-Proven Vasculitic Peripheral Neuropathy Associated with Small Cell Carcinoma

R. Ganesan, P. Matthews and R. McKelvey (Montreal, Quebec)

Paraneoplastic vasculitic neuropathies have been previously reported in case studies and small series. We report a case of a man with a biopsy-proven vasculitic polyneuropathy whose initial symptoms manifested 30 months prior to diagnosis of a small cell carcinoma of the lung.

A 54-year-old man presented with a 30 month history of progressive leg weakness, resolving abducens palsies, left arm dystonia and new onset paroxymal oropharyngeal movements with preservation of consciousness.

Investigations on admission included a lumbar puncture positive for oligoclonal bands, and showing elevated protein; an EMG showing widespread denervation of arm and leg muscles; nerve conduction studies showing sensory and motor conduction slowing; MRIs (with and without gadolinium) of the spine and head showing no abnormality; and a nerve-muscle biopsy showing active Wallerian degeneration and epineural vasculitis. Subsequent biopsy of a left lung mass confirmed small cell carcinoma. Immunological markers in the serum and CSF were also studied.

This case supports the association of vasculitic peripheral neuropathy and malignancy. Further studies may identify the precise immunological basis of association. 
P129.

\section{Studies of Pudendal Nerve Function in Faecal Incontinence}

T.G. Staunton and B. Stanley (Oakville, Ontario)

Faecal incontinence without significant bladder dysfunction or other neurologic disease represents a bilateral fascicular pudendal neuropathy. Typically this occurs in females with a history of both obstetric trauma and chronic constipation. It is postulated that the local trauma to the fascicles of the pudendal nerve to the external anal sphincter are preferentially affected by difficult childbirth and exacerbated by the traction effect of pelvic descent from chronic straining at stool; there is usually preservation of the fascicles that supply the levator ani/puborectalis and the urethral sphincters. We describe a series of patients upon whom we performed standard nerve conduction, Pudendal nerve latency (using St. Mark's Pudendal electrode) and E.M.G. studies of the external anal sphincter and puborectalis, to define this focal neuropathy. Pudendal nerve and sphincteric function has direct bearing on surgical and other therapies for faecal incontinence, as they help to distinguish between neurogenic incontinence and sphincteric disturbance due to muscular dehiscence or cicatrix, which have significantly different prognosis.

\section{P130.}

\section{An Atypical Motor Neuron Disease Syndrome in Patients} with Breast Implants

\section{B. Ostermeyer ShoAib and B.M. PATten (Houston, Texas)}

Six women developed motor neuron disease syndrome (MNDS) at a median latency of 11 years (range $2-23$ years) after receiving silicone-gel breast implants $(n=5)$ and saline breast implants $(n=1)$. At explantation in five, all had ruptured implants with silicone spilled into tissue. In addition to the MNDS, patients had myalgia, fatigue, arthralgia, joint swelling and stiffness, rash, headache, Sjorgren's syndrome or Raynaud's phenomena. Three patient died (two 7, one 8 years after onset of symptoms) during the study. Some had autoantibodies such as anti-GM1, ANA or antimyelin antibodies or abnormal serum levels of immunoglobulins. Biceps muscle biopsy was done in five patients and showed neurogenic atrophy in all. Foreign material such as silicone breast implants might cause a MNDS probably indirectly through autoimmune mechanism. Further investigations of the syndromic nature of MND associated with silicone breast implants are needed.

P131.

\section{Sacral Plexopathy Complicating X-linked Hypogammaglo- bulinemia}

\section{B.J. HoPPE and V.A. CWIK (Edmonton, Alberta)}

We report a 21 -year-old male, identical twin, with X-linked hypogammaglobulinemia, who suddenly developed a left sacral plexopathy. Extensive investigation revealed metastic adenocarcinoma, of unknown primary origin, with widespread abdominal and pelvic involvement of both bone and soft tissue. A large, lobulated mass $(10 \times 5 \times 6 \mathrm{~cm})$, arising from the left side of the sacrum and extending into the left pelvis, is responsible for his neurological symptoms and findings.

Lumbosacral plexopathy, secondary to a neoplastic lesion, is most commonly due to invasive colorectal, uterine or prostate cancer, but may also be secondary to metastastic spread from a variety of neoplasms. Primary immunodeficiency disorders predispose to malignancy, particularly GI tract and leukemia. The incidence of rectosigmoid cancer in patients with $\mathrm{X}$-linked agammaglobulinemia has been calculated to be 30 times higher than normal.

To our knowledge, no other case of primary immunodeficiency syndrome, complicated by malignancy causing lumbosacral plexopathy, has been reported. The twin brother also has $\mathrm{X}$-linked hypogammaglobulinemia, but is apparently free of malignancy at this time.

\section{P132.}

\section{Uremic Ischemic Myopathy}

C.A.J. Boyle, J. Ravindran, A. Shuaib, D. George, M.A. BALTZAN and S. KWAUK (Saskatoon, Saskatchewan)

Myopathy in renal failure is most commonly seen due to cachexia, polneuropathy, steroids or water and electrolyte disturbance. However, painful proximal ischemic myopathy, in association with renal failure and hyperparathyroidism, is rare, having only been documented histologically twice previously in the literature. A third case is hereby presented accompanied by detailed clinical information and discussion of the pathology of a series of muscle biopsies. This report details a 37 -year-old man in acute kidney transplant rejection with hyperparathyroidism who presented with a two week history of severe bilateral quadriceps myalgias and weakness. CK was $5100 \mathrm{U} / \mathrm{L}$. Despite dialysis and a surgical parathyroidectomy, the muscle pain continued to be severe, requiring medical pain management with morphine, carbemazepine and amitriptyline. Respiratory failure necessitated ventilation. He required debridement of extensively necrosed muscles of both thighs, and subsequently developed multiple areas of skin necrosis. EMG initially showed some mild neuropathy and later some myopathic changes. Serial muscle biopsies showed that he had a progressive noninflammatory myopathy associated with ischemic infarction of the muscle with extensive dystrophic calcification. This diagnosis should be considered when patients in renal failure present with painful proximal myopathy with persistant elevation of the $\mathrm{CK}$.

P133.

Cardiomyopathy in Association with Adult Onset Nemaline Myopathy

J. Ravindran, J.R. Donat, C. Wells, M. Khouri and D. GEORGE (Saskatoon, Saskatchewan)

Adult onset Nemaline myopathy (NM) is a rare disease. Cardiomyopathy has been reported with congenital rapidly progressive or non-progressive and asymptomatic NM but not with adult onset NM. We report a 43-year-old man with adult onset NM who also had cardiomyopathy with myocardial biopsy negative for Nemaline rods. He presented with a history of proximal 
muscle weakness of one year duration. Examination revealed moderate weakness of proximal muscles of upper and lower extremities and neck muscles. Muscle biopsy was positive for nemaline rods with paucity of inflammatory cells. CPK was elevated but he had no response to a trial of steroids. Investigations for other causes of polymyositis were negative. Echocardiography and cardiac catheterization findings were suggestive of dilated cardiomyopathy. Myocardial biopsy was negative for nemaline rods. Previous case reports of cardiomyopathy in association with congenital NM are reviewed. The cause of CPK elevation in this case similar to one earlier case report is unknown. The relationship of nemaline rods to the severity and mechanism of muscle weakness is not clear. Patients with NM should be screened for associated Cardiomyopathy.

\section{P134.}

\section{Dropped Head Syndrome: Clinical and Histological Findings in One Patient}

F. Grand'Maison, Z. NASREddine, M.L. Lebel and J.-P. Bernier (Sherbrooke, Quebec)

The "dropped head syndrome" was recently described in four patients with weakness restricted to neck extensor muscles. Paraspinal muscle biopsy showed non-specific myopathic changes with an increase in fibre size variability and a motheaten appearance of occasional fibres. It was concluded that the dropped head syndrome represents a restrictive myopathy of unknown etiology. We report a patient with clinical features typical of DSH in whom additional pathological changes were seen.

A 49-year-old schizophrenic man complained of neck discomfort followed by severe weakness of neck extensor muscles over the next three weeks. Weakness remained stable over the next three months. He had no other neurological complains or systemic symptoms. Neurological examination confirmed that weakness was limited to the neck extensors with possible mild involvement of sternomastoid muscles. The $\mathrm{CK}$ level was slightly elevated. EMG showed fibrillation potentials and myopathic changes essentially limited to cervical paraspinal muscles.

Paraspinal muscle biopsy revealed an increase in fibre size variability. NADH reactive sections showed not only occasional moth-eaten fibres but also abundant core-targetoid fibres. This additional features may help in the nosological classification of this rare neuromuscular condition. ISuarez GA, Kelley JJ Jr. The dropped head syndrome. Neurology 1992; 42: 1625-
1627.

\section{P135.}

Winging of the Scapula Caused by a Muscle Contracture in a Neurologically Normal Individual

\section{G.S. TARDIF (Ottawa, Ontario)}

Winging of the scapula is a common reason for referral to an EMG laboratory. Most cases are secondary to lesions of the long thoracic nerve. Other neuromuscular lesions can also be responsible for winging. This presentation will feature a healthy young individual with a normal neuromuscular and electrodiagnostic examination. A more detailed musculoskeletal examination revealed that a muscle contracture was responsible for the scapular winging. The biomechanics underlying this particular problem with be dicussed.

P136.

\section{Myoblast Tansplantation in Immunologically Favorable Situations}

J.-P. Bouchard, J.P. Tremblay, F. Malouin, R. Roy, I. Kinoshita, J.T. Vilquin, J. HuARD, B. GuÉretTE and I. ASSElin (Quebec, Quebec)

Myoblasts were transplanted in immunodeficient (nude and SCID) and in immunocompetent ( $\mathrm{mdx}$ ) mice with or without immunosuppression to assess the role of the immune system in the success or failure of such transplantation. Myoblasts for these transplantations were either of human origin or from a transgenic mice expressing $\beta$-galactosidase under a muscle promoter. The injected muslces were irradiated and damaged with notexin before the transplantation. In SCID mice a high percentage $(90 \%)$ of the muscle fibers expressed human dystrophin (mAb NCLDys3) or $\beta$-gal. These markers were not observed following transplantation in $\mathrm{mdx}$ mice without immunosuppression. With or without immunosuppression, the injected muscle was infiltrated with $\mathrm{CD}^{+}, \mathrm{CD}^{+}$or IL-2R $\mathrm{T}^{+}$cells. A high percentage of muscle fibers expressing $\beta$-gal and dystrophin were, however, observed in $\mathrm{mdx}$ mice immunosuppressed with rapamycin or FK506. the presence of normal dystrophin mRNA in these transplanted mdx muscles was demonstrated by PCR.

A successful myoblast transplantation was also obtained between identical twin girls both carriers of Duchenne muscular dystrophy. The strength of the symptomatic carrier was significantly $(12-31 \%)$ increased in a single muscle by transplantation of myoblasts obtained from the asymptomatic twin.

Our results emphasize the importance of the immune system in the success or failure of myoblast transplantation.

\section{P137.}

\section{Cylindrical Spirals in Skeletal Muscles}

J.-P. Bouchard, F. Gagne, S. Tremblay and D. Brunet (Quebec, Quebec)

The description of cylindrical spirals in skeletal muscle came from Canada in 1979 (Carpenter et al.). The clinical picture associated with these findings includes muscle pain, cramps, weakness, and gait disorders. We wish to add a tenth case to the medical literature.

A 44-year-old male complained of fatigue, sudation and occasional myalgia. There were no cramps, weakness, numbness or ataxia. CK was either normal or elevated in four instances $(550 ; 2623 ; 3349 ; 308 \mathrm{U} / \mathrm{L})$. The following tests were all normal: hematology, cortisol and TSH, serology for EBV and $\mathrm{CMV}$, serum ammonium and lactate at rest and after forearm exercise, EMG.

Muscle biopsy from the left biceps revealed numerous clusters of rod-like structures generally orientated according to the long axis of otherwise well preserved Type II fibers. The rods were stained red by the modified trichrome reaction. In transverse EM sections the rods appeared as multilayered cylindrical tubes centered by a thin area rich in glycogen granules. Longitudinal 
sections showed the continuity of these central tubes with the adjacent sarcoplasm. In some fibers the sarcoplasmic reticulum was dilated.

The significance of cylindrical spirals in skeletal muscles has yet to be enlightened.

\section{P138.}

\section{Spontaneously Remitting Severe Idiopathic Generalized} Myokymia

\section{P. PATHAK and P.R. BouRque (Ottawa, Ontario)}

Objective: To report a case of subacute generalized myokymia with documented motor nerve hyperexcitability and spontaneous remission.

Background: Secondary generalized myokymia has been described with chronic acquired polyneuropathy, Guillain-Barré syndrome, underlying malignancy, thyrotoxicosis and toxic exposure (timber rattlesnake, gold, penicillamine). Some idopathic cases may respond to plasmapheresis. Other cases are non progressive and may have a genetic cause.

Results: This 70-year-old previously healthy man presented with muscular pain and diffuse weakness. Examination revealed mild diffuse paraparesis $(4+/ 5)$ and hyporeflexia. There was marked generalized myokymia, best seen after brief exertion. Investigations for metabolic or autoimmune disease and underlying malignancy were negative. Motor nerve conduction studies showed generalized hyperexcitability manifested in $50-75 \mathrm{~ms}$ after discharges following single stimulation. Motor conduction velocities and amplitudes and sensory conduction studies were normal. EMG showed prominent myokymic discharges (intraburst frequency $20-60 \mathrm{~Hz}$, most recurring semi-rhythmically at $0.5-5 \mathrm{~Hz}$ ). Carbamazepine was tried briefly but poorly tolerated. Disability peaked at 6 weeks, followed by spontaneous recovery in 4 months.

Conclusions: This case showed features of both idiopathic generalized myokymia and "cramp-fasciculation syndrome". The subacute evolution and spontaneous remission strongly favor a toxic or autoimmune etiology.

\section{P139.}

\section{Physostigmine Reverses the Lexical Priming Impairment} Observed in Myasthenia Gravis

\section{J.-P. Bouchard, C. Truchon, J.J. Coté and J. Doyon (Quebec, Quebec)}

We recently reported that, compared to control subjects, patients with myasthenia gravis demonstrate a severe deficit in lexical priming (Bouchard et al., 1993). Other impairments in auditory vigilance, verbal fluency and immediate visual memory were also observed, hence supporting the hypothesis of a central cholinergic dysfunction in myasthenic patients.

In the present study, we investigated the effects of physostigmine (a cholinergic agonist crossing the blood-brain barrier) on cognitive functions in myasthenia gravis. Three myasthenic patients who had showed a severe deficit on the lexical priming task participated in this research project. These subjects were tested twice, five weeks apart, on equivalent versions of tests measuring primary memory (Digit Span), secondary memory (Logical Memory and Designs Reproductions of the Wechsler Memory Scale), lexical priming, auditory vigilance and frontallobe functions (Self-Order Pointing and Conditional Associative Learning tasks). The first session was preceeded by an intravenous injection of $1 \mathrm{mg}$ of physostigmine and $0.2 \mathrm{mg}$ of glycopyrrolate.

Physostigmine significantly reduced or even eliminated the lexical priming impairment, whereas performance returned to baseline level five weeks later. Physostigmine also produced a severe impairment on the Conditional Associative Learning task. These findings provide the first demonstration that the lexical priming deficit in myasthenic patients can be reversed pharmacologically.

\section{II-7. GENERAL NEUROLOGY}

P140.

Is There Long Term Benefit after Dihydroergotamine Therapy for Chronic Daily Headaches?

C.A.J. Boyle, N. Sharif and A. Shuaib (Saskatoon, Saskatchewan)

Chronic intractable headaches are notoriously difficult to treat effectively and with prolonged efficacy. Overuse of analgesics can themselves contribute to chronic daily headaches by causing rebound pain. Intravenous dihydroergotamine (DHE) therapy has proven effective in tapering patients from chronic medication usage. The short term benefits have been well documented but little is written about the longer term benefits. 30 female and 8 male patients with chronic daily headaches were treated following a modification of the Raskin protocol with intravenous DHE and metoclopramide initially then conversion to subcutaneous DHE and a phrophylactic agent. 26 patients were receiving analgesics and narcotics at the time of admission. 33 patients improved. The study was done with a retrospective hospital chart review for the effects and side effects of the drug at the time of therapy. 26 of 33 patients discharged on DHE were later contacted by telephone. 21 of these patients had improvement in their symptoms, remaining on a prophylactic agent with DHE as needed, but no longer requiring narcotics. In the management of intractable headaches, this study confirms that an in-hospital course of DHE results in a favourable outcome in the short term and the longer term effectiveness in decreasing dependence on analgesic medications.

\section{P141.}

Familial Hemiplegic Migraine with Migraine Coma: An Autosomal Dominant Disorder with a Possible Defect in Cerebral Energy Metabolism

B. Zifkin, F. Andermann, E. Andermann and D.L. Arnold (Montreal, Quebec)

A mother and son with familial hemiplegic migraine (FHM), nystagmus, and tremor developed stupor, at times with meningeal signs during their attacks. With recovery of consciousness, they became agitated and developed hallucinations 
suggesting peduncular hallucinosis. Attacks were not fully prevented by propranolol or pizotyline. Blood alanine levels were high, and pyruvate increased during an attack. Lactate was not increased in blood or on ${ }^{1} \mathrm{H}$ brain magnetic resonance spectroscopy.

This autosomal dominant syndrome has been described as migraine coma, and has been lethal in some patients. It is at present not clear whether all dominantly inherited hemiplegic migraine or lethal migraine is a manifestation of this disorder, which may be due to a defect in cerebral energy metabolism.

\section{P142.}

\section{Chorea as a Presenting Feature of Alzheimer's Disease}

J. Ravindran, B.J. Stewart, P. Siemens, A.H. Rajput and B. RozDILSKI (Saskatoon, Saskatchewan)

Choreic manifestations may be seen with a wide variety of anatomical lesions and metabolic disorders. Several manifestations of extra-pyramidal dysfunction, including parkinson-like syndrome have been reported during the course of Alzheimer's disease. One case report suggested that gamma aminobutyric acid (GABA) agonist Baclofen triggered generalized chorea in Alzheimer's disease. We report a patient with Alzheimer's disease in whom the major presentation was chorea. A 50-year-old lady presented with dementia and chorea involving face, limbs and tongue. Huntington's disease was diagnosed. She died at the age of 62 and autopsy revealed pathologic changes suggestive of Alzheimer's disease with no evidence of Huntington's disease. Neurofibrillary tangles occur in substantia nigra in Alzheimer's disease but spontaneous chorea in Alzheimer's disease has not been reported. One possible mechanism is decreased GABA-ergic inhibition of globus pallidus interna output to ventrolateral thalamus which innervates premotor cortex resulting in chorea.

P143.

Trends in Mortality Attributed to Alzheimer's Disease in Canada

L.W. Svenson, R.L. Campbell and R.B. Faulkner (Edmonton, Alberta)

Using data from the Centre for Health Information of Statistics, the number of deaths attributed to AD between 1980 and 1989 were examined by age, sex and province. Mortality rates were age-standardized using the direct method to the 1981 Canadian population. Mortality rates varied across provinces with the highest rates found in the eastern-most provinces. Examination of standardized mortality ratios (SMRs) found that males in Newfoundland, Prince Edward Island and Ontario had an excess risk of having $\mathrm{AD}$ recorded on the death certificate, relative to Canada as a whole. The four western-most provinces and Nova Scotia all had a statistically significantly low risk of having AD recorded as the cause of death. Females had a similar pattern in Newfoundland, New Brunswick and Ontario showing an excess risk of mortality, and only Manitoba, Saskatchewan and Alberta having a significantly low risk. All ten provinces displayed increases in mortality due to AD over the study period. Rates for Canada increased from 0.94 and 0.69 per 100,000 for males and females respectively in 1980 to 7.4 and 6.5 in 1989. The increase in mortality rates may reflect an increase in the incidence and/or prevalence of $\mathrm{AD}$, or may reflect an increased willingness of physicians to record $\mathrm{AD}$ as the underlying cause of death.

\section{P144.}

\section{Reversible Primitive Reflexes in Dementia of Depression: A Case Report}

\section{J.C. HostetLeR and D.G. Brunet (Kingston, Ontario)}

Release of primitive reflexes in adults is generally considered to be associated with organic neurologic disease of the frontal lobes or related structures. The authors describe a case of a patient with reversible dementia of depression and striking primitive reflexes, including suck and bilateral grasp reflexes; in which the cognitive and affective disorder, as well as the primitive reflexes, resolved after tricyclic antidepressant therapy. Literature on the role of primitive reflexes in neuropsychiatric disorders is reviewed and the significance of the authors' case of concepts of the neurologic basis of dementia of depression is discussed.

P145.

Intrathecal Administration of a Selected Synthetic Peptide of Human Myelin Basic Protein to Patients with Chronic Progressive Multiple Sclerosis

\section{K.G. WARREN and I. CATZ (Edmonton, Alberta)}

The great majority of patients with chronic progressive multiple sclerosis (CPMS) have increased levels of autoantibodies to myelin basic protein (anti-MBP) in both free (F) and bound (B) forms in their cerebrospinal fluid (CSF) and central nervous system (CNS) tissue. Previous in vitro research has shown that anti-MBP can be neutralized by a selected synthetic peptide corresponding to residues 75-95 of human MBP (synthetic peptide residues 75-95) are not by synthetic peptide residues $35-58$. The purpose of this study was to determine if these results from in vitro experiments are also observed in in vivo studies. Increasing amounts of these two synthetic peptides were inoculated intrathecally into patients with advanced CPMS and titers of F and B CSF anti-MBP were monitored prior to inoculation (baseline), at half our intervals for the next two hours and 24 hours later. $F$ anti-MBP was neutralized by synthetic peptide residues 75-95 in a dose dependent manner and not by synthetic peptide residues 35-58. Pharmacokinetic results of this study will be presented. If anti-MBP is involved in the mechanism of MS demyelination it can be blocked or neutralized by selected synthetic peptides.

P146.

Signal Transduction Mechanisms in Multiple Sclerosis: Phospholipase $\mathbf{A}_{2}$

J.R. Wherrett, S.J. Huterer and W.W. Tourtellotte (Toronto, Ontario; Los Angeles, California)

Phospholipases serve as central regulatory steps in signal transduction and in generation of inflammatory mediators. We 
have begun to assess the involvement of phospholipases $A_{2}$ $\left(\mathrm{PLA}_{2}\right)$ in the immune-inflammatory mechanisms occurring in MS by assaying "secretory", "lysosomal" and "cytosolic" activities directly in post-mortem tissue using arachidonyl-labelled micellar and E. coli substrates. Activity of "secretory" enzyme in plaque $(24.5 \pm 7.0 \mathrm{nmol} / \mathrm{h} / \mathrm{g}$ wet $\mathrm{wt})$ and normal-appearing white matter (NAWM) $(27.2 \pm 6.1)$ was similar to control $(26.3$ $\pm 8.1)$, whereas "lysosomal" was elevated in plaque $(7.2 \pm 2.1, \mathrm{p}$ $<0.01)$ and NAWM $(8.5 \pm 2.8, \mathrm{p}<0.005)$ over control $(4.7 \pm$ $0.7)$ and "cytosolic" was decreased in plaque $(18.9 \pm 6.2, \mathrm{p}<$ $0.0005)$ and NAWM $(16.0 \pm 2.1, \mathrm{p}<0.0005)$ compared to control $(38.1 \pm 6.0)$. "Cytosolic" activity was also lower in MS cerebral cortex $(11.3 \pm 8.5, \mathrm{p}<0.0125)$ than in control cortex (26.9 \pm 9.2$)$. Identity of most of the "cytosolic" activity with the $85 \mathrm{kDa}$ enzyme purified from human monocytes was confirmed by FPLC gel filtration and immuno-precipitation. Elevated activity of "lysosomal" PLA ${ }_{2}$ as found previously for other lysosomal enzymes could reflect lysosomal proliferation in macrophages and other cells. The marked decrease of "cytosolic" $\mathrm{PLA}_{2}$ in MS brain which does not correlate with degree of demyelination suggests loss of a cellular source, excessive proteolysis in MS or a dysregulation.

\section{P147.}

Ethnic Origin and Risk of Multiple Sclerosis (MS): Descriptive and Case-Control Data on Parental Ancestry in Male and Female MS Patients from Alberta, Canada

S.A. Warren, K.G. Warren, L. Svenson and S. Woodhead (Edmonton, Alberta)

Descriptive studies of US male veterans have implicated Scandinavian ancestry as an MS risk factor; but neither descriptive studies of females, nor case-control studies, on ethnicity have been published. Population ancestry data and MS prevalence rates provided by Alberta Health were correlated throughout Alberta's 19 census divisions, for males and females separately; and 276 patients ( 86 males $/ 190$ females) who attended the University of Alberta MS Clinic were compared on ethnic origin to sex-matched friends and nonblood relatives. Percentage of population reporting Scandinavian ancestry on both parents' side was associated with MS prevalence in men only $(r=+.45, p$ $<.05$ ). Case-control comparisons, again with people reporting single ancestry, failed to find such a relationship in men or women. However there was an excess relative risk (RR) of MS associated with nonspecific European as opposed to British ancestry for men $(\mathrm{RR}=3.0,95 \% \mathrm{CI}=1.1-8.2, \mathrm{p}<.05)$, along with a nonsignificant trend in women. When paternal vs. maternal ancestry was compared, there was an excess MS risk associated with having a father of European as opposed to British ancestry for both men and women $(\mathrm{RR}=2.7,95 \% \mathrm{CI}=1.3-5.6, \mathrm{p}<.01$ and $\mathrm{RR}=1.8,95 \% \mathrm{CI}=1.1-2.9, \mathrm{p}<.05)$. The case-control data suggests that the descriptive association between Scandinavian origin and MS is an "ecological fallacy" and that fathers are me likely to transmit a risk factor underlying ethnicity.
P148.

The Prevalence and Geographic Distribution of Multiple Sclerosis (MS) in Alberta: A Population-Based Study Using Provincial Health Care Records

L.W. Svenson, S.E. Woodhead, S.A. Warren and G.H. Platt (Edmonton, Alberta)

MS prevalence was estimated for each of the 19 census divisions (CDs) of Alberta, by age and sex using data from the Alberta Health Care Insurance Plan (AHCIP). A cohort of all AHCIP registered individuals (2.4 million) was extracted and followed for five years with all individuals receiving a service for MS extracted. The overall crude prevalence was 217 per 100,000 ( 173 for males; 260 for females) with a mean age of 41 years ( 42 for males; 40 for females). Both sexes were found to have a statistically significant variation across CDs. Prevalence per 100,000 population ranged from a low of 64 for males in CD 17 to a high of 404 for females in CD 7. Males appeared to have a higher prevalence in the more rural CDs while females had similar rates in both urban and rural areas. The northern-most CDs had the lowest prevalence for both sexes. Females had their highest prevalence in the southern parts of the province while males had their highest prevalence in the north-central areas. The areas with the lowest prevalence also had the highest proportions of aboriginal residents. Prevalence estimates using health care records were similar to community-based studies conducted in Alberta which supports the validity of the geographic distribution noted.

\section{P149.}

HTLV-1 Associated Myelopathy in Natives of British Columbia: a Point-Epidemy in $\mathrm{BC}$ or a Case of Missed Diagnosis Elsewhere?

J.-F. Oger and G. Dekaban (Vancouver, British Columbia; Toronto, Ontario)

HTLV-1 associated Myelopathy (HAM) is endemic in Japan, Western Indies and Africa. In Canada it is found among immigrants from Western Indies (ref). We have identified $6 \mathrm{BC}$ Natives with HAM. They had a paraparesis with motor deficit and spasticity, chronic urinary retention, sensory abnormalities in the legs and prominent painful dysesthesia. One patient had a 20 year history but most had subacute disease $(<2$ years $)$. CSF revealed lymphocytes $\left(8\right.$ to $60 / \mathrm{mm}^{3}$ ) and increased proteins with high IgG and oligoclonal bands. Some had MRI abnormalities and this lead to the diagnosis of multiple sclerosis in 2. Diagnosis was suspected by ELISA (HTLV-1/2 positivity, CDC). Confirmation was obtained by PCR using HTLV-1 specific probes. PCR was positive on the single CSF examined where HTLV-1 was rescued from 20,000 cells. Risk factors included blood transfusion $(n=2)$, needle sharing $(n=2)$, sexual activity $(n=2)$ but also vertical transmission from a mother with a leukemic disease $(n=1)$. Familial aggregates of HTLV-1 positivity was found with evidence for vertical and commensal transmission. Canadian Neurologists need to become familiar with this disease as it could be the tip of the iceberg of an endemic problem in Canadian populations with consequences for Public Health, STD prevention and Blood Transfusion. 